\title{
\#USGS
}

science for a changing world

Prepared in cooperation with the Elbert County Board of County Commissioners

\section{Groundwater-Level Elevations in the Denver Basin Bedrock Aquifers of Elbert County, Colorado, 2015-18}

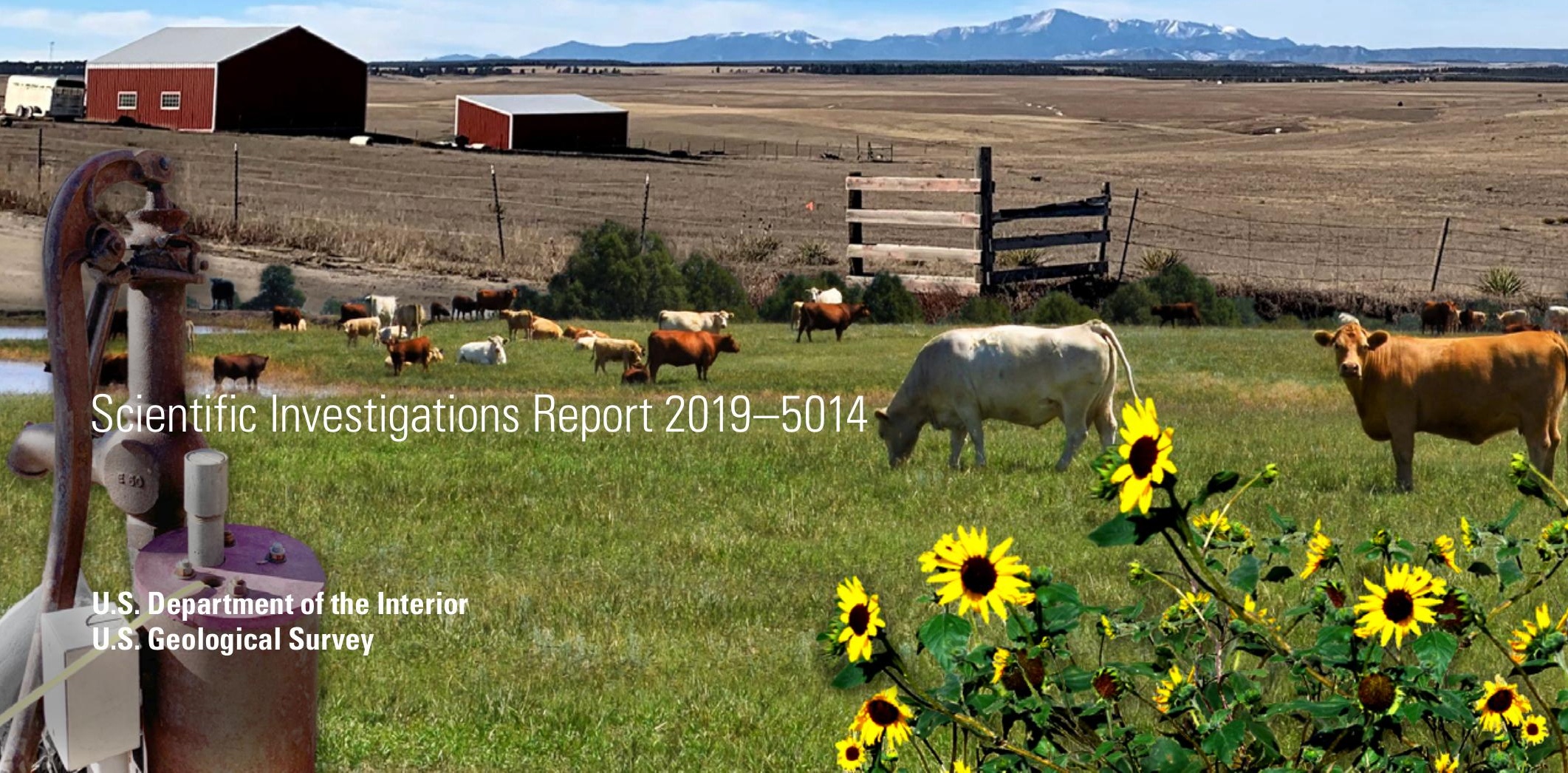


Cover. Cover is a montage of photographs taken in Elbert County, Colorado, between August and December 2018. Photographs by C.A. Penn, U.S. Geological Survey. 


\section{Groundwater-Level Elevations in the Denver Basin Bedrock Aquifers of Elbert County, Colorado, 2015-18}

By Colin A. Penn and Rhett R. Everett

Prepared in cooperation with the Elbert County Board of County Commissioners

Scientific Investigations Report 2019-5014 


\title{
U.S. Department of the Interior \\ DAVID BERNHARDT, Secretary
}

\author{
U.S. Geological Survey \\ James F. Reilly II, Director
}

U.S. Geological Survey, Reston, Virginia: 2019

For more information on the USGS - the Federal source for science about the Earth, its natural and living resources, natural hazards, and the environment-visit https://www.usgs.gov or call 1-888-ASK-USGS.

For an overview of USGS information products, including maps, imagery, and publications,

visit https://store.usgs.gov.

Any use of trade, firm, or product names is for descriptive purposes only and does not imply endorsement by the U.S. Government.

Although this information product, for the most part, is in the public domain, it also may contain copyrighted materials as noted in the text. Permission to reproduce copyrighted items must be secured from the copyright owner.

Suggested citation:

Penn, C.A., and Everett, R.R., 2019, Groundwater-level elevations in the Denver Basin bedrock aquifers of Elbert County, Colorado, 2015-18: U.S. Geological Survey Scientific Investigations Report 2019-5014, 50 p., https://doi.org/10.3133/sir20195014.

ISSN 2328-0328 (online) 


\section{Acknowledgments}

The authors would like to thank the residents of Elbert County, especially the well owners who allowed access to their property. The authors also acknowledge the participation of the Elbert County Board of County Commissioners and Water Advisory Council. The U.S. Geological Survey and Elbert County Board of County Commissioners acknowledge the Colorado Water Conservation Board for partially funding the study. The authors also acknowledge U.S Geological Survey colleagues Evan Gohring and Jordan Skipwith for assistance with collecting and processing data related to this study.

\section{Contents}

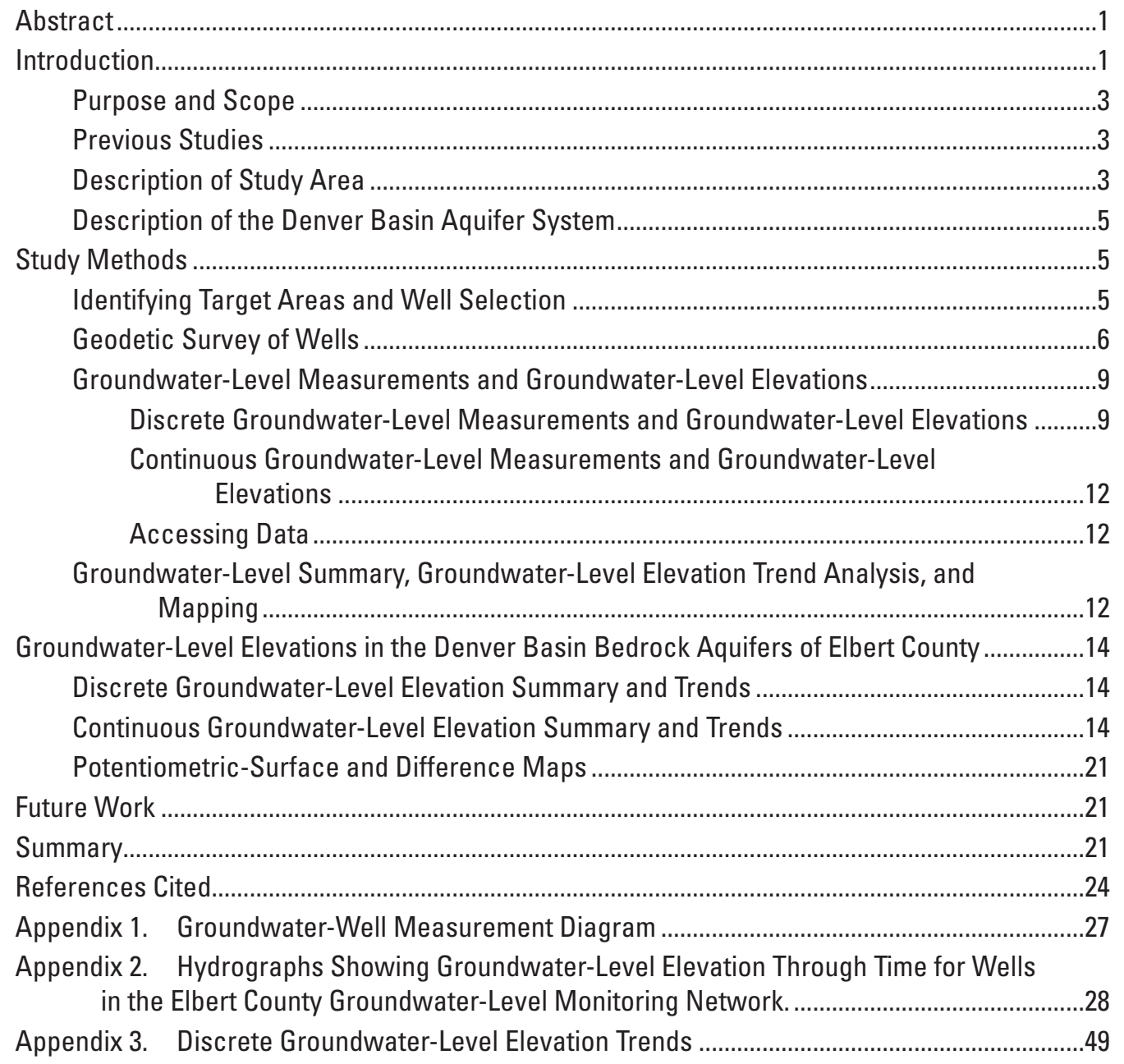




\section{Figures}

1. Location of the Denver Basin aquifer system and geologic lines of section $A, A-A^{\prime}$ and $B, B-B^{\prime}$ near Elbert County, Colorado

2. Location of groundwater-level monitoring network wells with aquifer of completion, Elbert County, Colorado. Well name abbreviations are as follows: UDAW, upper Dawson aquifer; LDAW, lower Dawson aquifer; DENV, Denver aquifer; ARAP, Arapahoe aquifer; LARA, Laramie-Fox Hills aquifer . .4

3. Generalized geologic cross sections $A, A-A^{\prime}$, west to east, and $B, B-B^{\prime}$, south to north, through the Denver Basin aquifer system. See figure 1 for cross section locations and table 1 for bedrock aquifer descriptions within each geologic unit...........6

4. Location of wells completed in Denver Basin bedrock aquifers, predicted 100-foot drawdown, well density, and areas of interest in the Arapahoe aquifer, for groundwater-level monitoring in Elbert County, Colorado.

5. Distribution of significant trends in discrete groundwater-level elevations, by aquifer, Elbert County, Colorado...

6. Distribution of significant trends in discrete groundwater-level elevations in the upper Dawson and lower Dawson aquifers near the towns of Elizabeth and Kiowa, Elbert County, Colorado

7. Estimated potentiometric surface of the upper Dawson aquifer in April 2018 and change in hydraulic head between April 2015 and April 2018, western Elbert County, Colorado

8. Estimated potentiometric surface of the lower Dawson aquifer in April 2018 and change in hydraulic head between April 2015 and April 2018, western Elbert County, Colorado....

1.1. Diagram showing example measurement point and groundwater-level measurement using $A$, a calibrated steel tape with chalk, and $B$, a calibrated electrical tape

2.1. Groundwater-level hydrograph for DENV 17, USGS site number 390755104172501, Elbert County, Colorado .28

2.2. Groundwater-level hydrograph for ARAP 8, USGS site number 390800104172601, Elbert County, Colorado....

2.3. Groundwater-level hydrograph for LARA 7, USGS site number 390817104040301, Elbert County, Colorado.

2.4. Groundwater-level hydrograph for DAWMAS26, USGS site number 390935104301001, Elbert County, Colorado

2.5. Groundwater-level hydrograph for UDAW 19, USGS site number 391126104354701, Elbert County, Colorado....

2.6. Groundwater-level hydrograph for DAWMAS27, USGS site number 391148104294101, Elbert County, Colorado....

2.7. Groundwater-level hydrograph for ARAP 7, USGS site number 391208104053301, Elbert County, Colorado.....

2.8. Groundwater-level hydrograph for DENV 16, USGS site number 391257104173601, Elbert County, Colorado....

2.9. Groundwater-level hydrograph for LDAW 16, USGS site number 391502104273601, Elbert County, Colorado.

2.10. Groundwater-level hydrograph for DAWMAS22, USGS site number 391545104335401, Elbert County, Colorado....

2.11. Groundwater-level hydrograph for LARA 6, USGS site number 391609104014001, Elbert County, Colorado 
2.12. Groundwater-level hydrograph for LARA 5, USGS site number 391621104012001, Elbert County, Colorado ..

2.13. Groundwater-level hydrograph for ARAPMAS27, USGS site number

391740104072401, Elbert County, Colorado ...

2.14. Groundwater-level hydrograph for DENV 15, USGS site number 391811104140301, Elbert County, Colorado

2.15. Groundwater-level hydrograph for DENV 14, USGS site number 391821104270601, Elbert County, Colorado

2.16. Groundwater-level hydrograph for LDAW 15, USGS site number 391829104385301, Elbert County, Colorado

2.17. Groundwater-level hydrograph for ARAPMAS22, USGS site number 391834104205601, Elbert County, Colorado

2.18. Groundwater-level hydrograph for DAWMAS28, USGS site number 391848104261401, Elbert County, Colorado

2.19. Groundwater-level hydrograph for DENMAS05, USGS site number 391851104204501, Elbert County, Colorado

2.20. Groundwater-level hydrograph for DAWMAS16, USGS site number 391852104391301, Elbert County, Colorado

2.21. Groundwater-level hydrograph for UDAW 18 , USGS site number 391915104375001, Elbert County, Colorado

2.22. Groundwater-level hydrograph for UDAW 14, USGS site number 391924104374101, Elbert County, Colorado

2.23. Groundwater-level hydrograph for ARAP 6, USGS site number 391946104114501, Elbert County, Colorado

2.24. Groundwater-level hydrograph for LDAW 12, USGS site number 392058104364401, Elbert County, Colorado

2.25. Groundwater-level hydrograph for LDAW 14, USGS site number 392125104323701, Elbert County, Colorado

2.26. Groundwater-level hydrograph for UDAW 17, USGS site number 392130104341401, Elbert County, Colorado

2.27. Groundwater-level hydrograph for DAWMAS21, USGS site number 392131104351701, Elbert County, Colorado

2.28. Groundwater-level hydrograph for UDAW 12, USGS site number 392133104310201, Elbert County, Colorado

2.29. Groundwater-level hydrograph for UDAW 16, USGS site number 392203104342301, Elbert County, Colorado

2.30. Groundwater-level hydrograph for UDAW 15, USGS site number 392355104382001, Elbert County, Colorado

2.31. Groundwater-level hydrograph for ARAPMAS28, USGS site number 392400104150601, Elbert County, Colorado

2.32. Groundwater-level hydrograph for ARAP 5, USGS site number 392434104142701, Elbert County, Colorado

2.33. Groundwater-level hydrograph for LARA 3, USGS site number 392616103591001, Elbert County, Colorado

2.34. Groundwater-level hydrograph for LARA 4, USGS site number 392635103590001, Elbert County, Colorado

2.35. Groundwater-level hydrograph for LDAW 13, USGS site number 392724104341901, Elbert County, Colorado 
2.36. Groundwater-level hydrograph for UDAW 13, USGS site number 392856104393801, Elbert County, Colorado

2.37. Groundwater-level hydrograph for DENV 13, USGS site number 393012104310701, Elbert County, Colorado 46

2.38. Groundwater-level hydrograph for UDAW 11, USGS site number 393016104392601, Elbert County, Colorado 46

2.39. Groundwater-level hydrograph for ARAP 4, USGS site number 393225104073601, Elbert County, Colorado.

2.40. Groundwater-level hydrograph for DAWMAS19, USGS site number 393227104343401, Elbert County, Colorado

2.41. Groundwater-level hydrograph for ARAP 3, USGS site number 393251104073701, Elbert County, Colorado 48

2.42. Groundwater-level hydrograph for DENV 12, USGS site number 393350104151701, Elbert County, Colorado

\section{Tables}

1. Physical characteristics of bedrock aquifers in the Denver Basin aquifer system..........7

2. Well identification and location information, and a summary of discrete groundwater-level measurements from April 2015 to June 2018 in Elbert County, Colorado

3. Summary of discrete groundwater-level elevations, April 2015 to June 2018, Elbert County, Colorado.

4. Statistically significant trends in discrete static groundwater-level elevations, April 2015 to June 2018, Elbert County, Colorado

5. Statistical summary of continuous daily maximum groundwater-level elevations, January 2016 to June 2018, Elbert County, Colorado

6. Trends in continuous and discrete daily maximum groundwater-level elevations, 2015 to June 2018, Elbert County Colorado.

3.1. Trends in discrete static groundwater-level elevations, April 2015 to June 2018, Elbert County, Colorado 


\section{Conversion Factors}

U.S. customary units to International System of Units

\begin{tabular}{lcl}
\hline & Multiply & \multicolumn{1}{c}{ Bo obtain } \\
\hline & Length & \\
\hline inch (in.) & 2.54 & centimeter $(\mathrm{cm})$ \\
inch (in.) & 25.4 & millimeter $(\mathrm{mm})$ \\
foot (ft) & 0.3048 & meter $(\mathrm{m})$ \\
mile (mi) & 1.609 & kilometer $(\mathrm{km})$ \\
\hline & Area & \\
\hline square mile $\left(\mathrm{mi}^{2}\right)$ & 259.0 & hectare $(\mathrm{ha})$ \\
square mile $\left(\mathrm{mi}^{2}\right)$ & 2.590 & square kilometer $\left(\mathrm{km}^{2}\right)$ \\
\hline & Volume & \\
\hline million gallons (Mgal) & 3,785 & cubic meter $\left(\mathrm{m}^{3}\right)$ \\
\hline & Flow rate & \\
\hline million gallons per day (Mgal/d) & 0.04381 & cubic meter per second $\left(\mathrm{m}^{3} / \mathrm{s}\right)$ \\
\hline
\end{tabular}

Temperature in degrees Celsius $\left({ }^{\circ} \mathrm{C}\right)$ may be converted to degrees Fahrenheit $\left({ }^{\circ} \mathrm{F}\right)$ as

$$
{ }^{\circ} \mathrm{F}=\left(1.8 \times{ }^{\circ} \mathrm{C}\right)+32 .
$$

Temperature in degrees Fahrenheit $\left({ }^{\circ} \mathrm{F}\right)$ may be converted to degrees Celsius $\left({ }^{\circ} \mathrm{C}\right)$ as

$$
{ }^{\circ} \mathrm{C}=\left({ }^{\circ} \mathrm{F}-32\right) / 1.8 .
$$

\section{Datum}

Vertical coordinate information is referenced to the North American Vertical Datum of 1988 (NAVD 88).

Horizontal coordinate information is referenced to the North American Datum of 1983 (NAD 83).

Elevation, as used in this report, refers to distance above the vertical datum. 


\section{Abbreviations}

$\begin{array}{ll}\text { ARAP } & \text { Arapahoe aquifer } \\ \text { CDWR } & \text { Colorado Division of Water Resources } \\ \text { CWCB } & \text { Colorado Water Conservation Board } \\ \text { DENV } & \text { Denver aquifer } \\ \text { ECBOCC } & \text { Elbert County Board of County Commissioners } \\ \text { GNSS } & \text { Global Navigation Satellite System } \\ \text { LARA } & \text { Laramie-Fox Hills aquifer } \\ \text { LDAW } & \text { Lower Dawson aquifer } \\ \text { LSD } & \text { land-surface datum } \\ \text { MP } & \text { measuring point } \\ \text { NAD 83 } & \text { North American Datum of 1983 } \\ \text { NAVD 88 } & \text { North American Vertical Datum of 1988 } \\ \text { NWIS } & \text { National Water Information System } \\ \text { OPR } & \text { Observation-Prediction } \\ \text { OPUS } & \text { Online Positioning User System } \\ \text { PPR } & \text { Parameter-Prediction } \\ \text { RTK } & \text { Real-time Kinematic } \\ \text { UDAW } & \text { Upper Dawson aquifer } \\ \text { UTM } & \text { Universal Transverse Mercator } \\ \text { WGS 84 } & \text { World Geodetic System of 1984 } \\ \text { USGS } & \text { U.S. Geological Survey }\end{array}$




\title{
Groundwater-Level Elevations in the Denver Basin Bedrock Aquifers of Elbert County, Colorado, 2015-18
}

\author{
By Colin A. Penn and Rhett R. Everett
}

\section{Abstract}

Public and domestic water supplies in Elbert County, Colorado, rely on groundwater withdrawals from five bedrock aquifers in the Denver Basin aquifer system (lower Dawson, upper Dawson, Denver, Arapahoe, and Laramie-Fox Hills) to meet water demands. Increased pumping in response to regional population growth and development has led to declining groundwater levels in neighboring Douglas County. The U.S. Geological Survey, in cooperation with the Elbert County Board of County Commissioners, began a study in 2015 to monitor groundwater levels within Elbert County. The purpose of this study is to report on groundwater levels measured between April 2015 and June 2018, and analyze trends and changes in groundwater-level elevations throughout the county.

Discrete groundwater levels were measured at 42 wells within Elbert County. Six of those wells contained equipment to make and record continuous groundwater-level measurements at hourly intervals. All five aquifers had wells with a rise in groundwater-level elevation and wells with a decline in groundwater-level elevation, based on a relative change in groundwater-level elevation between the April 2015 and April 2018 measurements. All aquifers except the upper Dawson had more wells with significant negative trends in discrete groundwater-level elevations than significant positive trends; however, at least one well within the upper Dawson, lower Dawson, Arapahoe, and Laramie-Fox Hills aquifers had a significant positive trend. Wells screened in the lower Dawson aquifer consistently had the most significant negative trends, with an average trend of -1.96 feet per year (ft/year). The upper Dawson, Denver, Arapahoe, and Laramie-Fox Hills aquifers had average trends of $0.03 \mathrm{ft} /$ year, $-1.04 \mathrm{ft} /$ year, $-0.46 \mathrm{ft} /$ year, and $-0.65 \mathrm{ft} /$ year, respectively. Trends in continuous groundwater-level elevations were in agreement with significant trends in discrete groundwater-level elevations. Potentiometric-surface maps of the upper and lower Dawson aquifers for April 2015 and April 2018 show that differences in hydraulic head from the two measurement periods were greatest along the western part of Elbert County. Results of this study could guide future groundwater monitoring in the county and aid in longterm planning of water resources.

\section{Introduction}

Elbert County is a mostly rural county in eastern Colorado and part of the western extent of the U.S. Great Plains. Public and domestic water users in Elbert County rely on groundwater withdrawals from bedrock aquifers in the Denver Basin aquifer system (herein referred to as Denver Basin bedrock aquifers) to meet their water-supply demands. In 2010, estimated withdrawals were 0.67 million gallons per day $(\mathrm{Mgal} / \mathrm{d})$ for public supply and $1.76 \mathrm{Mgal} / \mathrm{d}$ for domestic supply, which are 13 percent and 33 percent, respectively, of total Elbert County groundwater withdrawals (5.27 Mgal/d; Maupin and others, 2014). Withdrawals for irrigation (45 percent), livestock ( 8 percent), and mining ( 1 percent) are the remaining Elbert County groundwater use. Population and corresponding water use has increased substantially in the county over the past 2 decades (1999-2018) and is expected to continue to rise in the future (Forsgren Associates Inc., 2018). The population of Elbert County from 1990 to 2000 increased from 9,646 to 19,872 (+106 percent), from 2000 to 2010 increased to 23,086 (+16 percent), and from 2010 to 2017 increased to 25,642 (+11 percent) (U.S. Census Bureau, 1996, 2000, 2017). Any continued increase in population, agriculture, or industry likely would lead to an increase in water demand that requires a proportional increase in water supply. Zoning regulations in the county stipulate that any new subdivision development must show 300 years of adequate water supply, including groundwater, as part of the permitting process (Elbert County, 2009).

Elbert County is surrounded by counties that also rely heavily on groundwater withdrawals from Denver Basin bedrock aquifers (fig. 1). To the west, Douglas County had an estimated 26.82 Mgal/d of groundwater withdrawals in 2010 (Maupin and others, 2014). Urban and suburban development in Douglas County has continued to increase, especially in areas that border Elbert County (Douglas County, 2018), putting further demand on groundwater resources in the area (Moore and others, 2007). The surrounding Arapahoe and El Paso Counties also rely on groundwater and withdrew an estimated $34.36 \mathrm{Mgal} / \mathrm{d}$ and $23.86 \mathrm{Mgal} / \mathrm{d}$, respectively, in 2010 (Maupin and others, 2014). Long-term groundwater monitoring in the Denver Basin bedrock aquifers by the Colorado Division of Water Resources (CDWR) indicates negative changes in groundwater-level elevations in all bedrock 


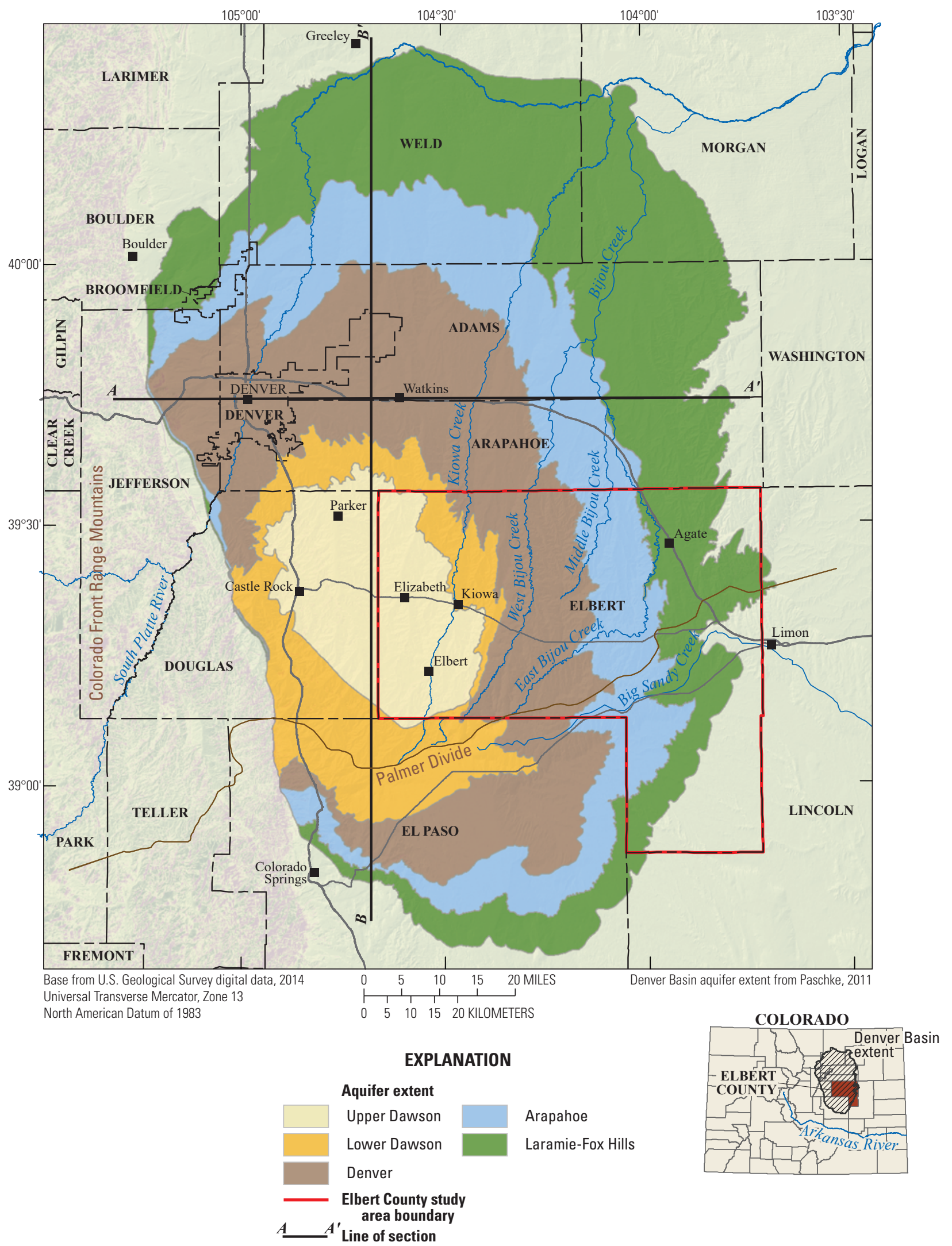

Figure 1. Location of the Denver Basin aquifer system and geologic lines of section $A, A-A^{\prime}$ and $B, B-B^{\prime}$ near Elbert County, Colorado. 
aquifers over the past 10 years (2007-17); however, more recent measurements (5- and 1-year changes) are inconsistent (Flor, 2017). Of the 290 wells monitored by CDWR in Denver Basin bedrock aquifers, 27 wells are in Elbert County.

Recognizing a need to better understand the future of water resources in Elbert County, the Elbert County Board of County Commissioners (ECBOCC) commissioned a comprehensive 5-year study of water resources and future demand scenarios (Forsgren Associates Inc., 2018). They also made a commitment to monitoring groundwater levels in the Denver Basin bedrock aquifers. The U.S. Geological Survey (USGS), in cooperation with ECBOCC, began a study in 2015 to measure groundwater levels in Denver Basin bedrock aquifers throughout Elbert County. The goals of this study were to establish a countywide groundwater-level monitoring network, make routine measurements of groundwater levels, and assess changes in groundwater-level elevations over the study period. Continuation of the monitoring network will aid in the longterm planning of water resources.

\section{Purpose and Scope}

The purpose of this report is to present an analysis of groundwater-level changes and trends in groundwater-level elevations in the Denver Basin bedrock aquifers in Elbert County, Colo., from April 2015 to June 2018. Forty-two wells are included in the monitoring network where discrete groundwater levels are measured bi-monthly (February, April, June, August, October, and December; fig. 2). At 6 of the 42 wells, a vented pressure transducer with an internal data logger records hourly groundwater-level measurements (also referred to as continuous groundwater levels). This report presents details of the well-selection process, a summary of groundwater levels measured during the study period, and a trend analysis of groundwater-level elevations in Elbert County.

\section{Previous Studies}

Groundwater levels in Elbert County historically have been measured by the Colorado Water Conservation Board (CWCB), CDWR, and USGS. McConaghy and others (1964) present measurements from 1956 to 1963 made by CWCB. Major and others (1983) report measurements from 1956 through 1981 made by USGS, and Flor (2017) more recently published groundwater levels measured by CDWR with varying periods of record through 2017. In neighboring Douglas County, a similar groundwater-level monitoring network, maintained by the USGS, has been operating since 2011 (Everett, 2014). Changes in groundwater levels, population growth, and water supply are topics of ongoing interest in Elbert County and the surrounding area (Moore and others, 2007; Forsgren Associates Inc., 2018).

Many previous studies have examined the geologic structure and hydrologic conditions of the Denver Basin bedrock aquifers and surrounding areas. These studies, listed or summarized in Wireman and Romero (1989) and Paschke (2011), were crucial to the development of a groundwater flow model (Robson, 1987) and a fully three-dimensional MODFLOW-2000 groundwater flow model (Paschke, 2011) of the Denver Basin aquifer system. The Kiowa Core, drilled on the Elbert County Fairgrounds in Kiowa, Colo., in 1999, provides a near-continuous record of geophysical properties for all bedrock aquifers in the Denver Basin aquifer system (Raynolds and others 2001). The 2,256-foot (ft) core was analyzed for hydrologic properties, leading to a better understanding of specific yield (Woodard and others, 2002) and hydraulic conductivity (Barkmann, 2004) in the basin, which helped to inform the MODFLOW-2000 model (Paschke, 2011).

\section{Description of Study Area}

Elbert County is a mostly rural, 1,850-square-mile $\left(\mathrm{mi}^{2}\right)$ county in eastern Colorado with an eastern border approximately 90 miles from the eastern border of Colorado. The northwestern corner of Elbert County is approximately 35 miles southeast of Denver, and the southwestern corner of the county is approximately 35 miles northeast of Colorado Springs (fig. 1). Neighboring counties are Arapahoe County to the north, Douglas County to the west, El Paso County to the southwest, and Lincoln County to the southeast and east. Natural vegetation is dominantly drought-resistant, high plains grasses that consist of Bouteloua gracilis (blue grama), Bouteloua dactyloides (buffalograss), Pascopyrum (wheatgrass), and Fescuta sp. (fescue). Ridgelines and rolling hills in the central and western parts of the county have large stands of Pinus ponderosa (ponderosa pine), and numerous riparian corridors support stands of Populus deltoides (cottonwood) and Salix, sp. (willow) (Elbert County Planning Commission, 2018). No perennial streams flow through the county; however, many intermittent streams originate in the county, including Kiowa Creek; East, Middle, and West Bijou Creeks, which flow to the South Platte River; and Big Sandy Creek, which flows to the Arkansas River.

The population of Elbert County has been increasing since the 1990 census. Rapid growth occurred from 1990 to 2000 , and more steady growth has occurred since 2000 . Western parts of the county are close to the urban corridor surrounding Denver and neighboring Douglas County urban centers, Castle Rock, and Parker. The 5-year water-supply study (Forsgren Associates Inc., 2018) focused on areas within the county expected to have the most development; these include the northwestern part of the county east of the city of Parker, called the Northwest Planning Area, and an area surrounding the towns of Elizabeth and Kiowa, called the Elizabeth-Kiowa Planning Area. The Elizabeth-Kiowa and Northwest Planning Areas are currently experiencing higher rates of growth and development than other parts of the county and include clustered development of subdivisions (Elbert County Planning Commission, 2018; Forsgren Associates Inc., 2018). 


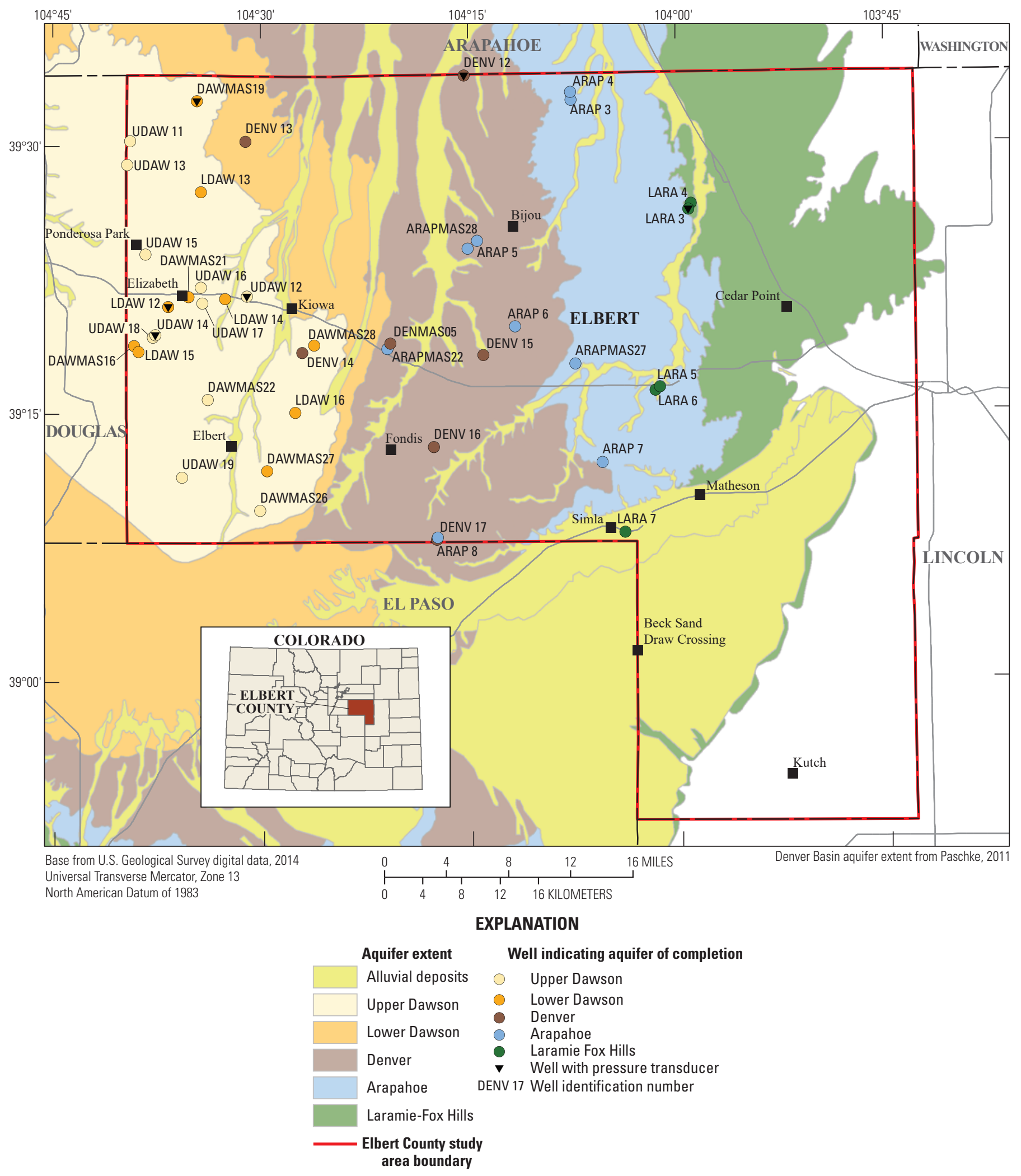

Figure 2. Location of groundwater-level monitoring network wells with aquifer of completion, Elbert County, Colorado. Well name abbreviations are as follows: UDAW, upper Dawson aquifer; LDAW, lower Dawson aquifer; DENV, Denver aquifer; ARAP, Arapahoe aquifer; LARA, Laramie-Fox Hills aquifer. 


\section{Description of the Denver Basin Aquifer System}

The Denver Basin aquifer system is bound on the western edge by the base of the Colorado Front Range and extends into the eastern plains of Colorado, covering an area of approximately 7,000 $\mathrm{mi}^{2}$ (fig. 1). The northernmost extent ends near the city of Greeley in Weld County, whereas the southern extent passes under the topographic Palmer Divide and ends southeast of Colorado Springs in El Paso County. The Denver Basin aquifer system is composed of several bedrock aquifers, which underlie Quaternary alluvial aquifers (Paschke, 2011). The bedrock aquifers in the basin have a synclinal structure composed of Late Cretaceous to Tertiary-age sandstone bedrock separated by unnamed claystone confining units (Fenneman, 1931; Robson, 1987; Paschke, 2011) and confined underneath by the Cretaceous Pierre Shale (fig. 3, table 1). The four bedrock aquifers, from oldest (deepest) to youngest (shallower), are the Laramie-Fox Hills aquifer in the Laramie Formation and Fox Hills Sandstone, Arapahoe aquifer in the Arapahoe Formation, Denver aquifer in the Denver Formation, and Dawson aquifer in the Dawson Formation. The Arapahoe and Dawson aquifers are divided by discontinuous confining units into upper and lower aquifers in parts of the basin. In Elbert County, the Dawson aquifer is divided into upper and lower aquifers. Parts of the basin are overlain by unconfined alluvial aquifers. Outcrops of each aquifer can be found along the outer edge of their extents and are generally considered unconfined, whereas confined conditions exist towards the interior of the basin in each aquifer where it is overlain by a younger confining unit (Paschke, 2011).

Many previous studies have examined the extent, thickness, age, and physical properties of each aquifer. The physical characteristics of the Denver Basin bedrock aquifers are summarized in table 1. Studies from which the information in table 1 was acquired include Romero (1976), Kirkham and Ladwig (1979), Schneider (1980), Robson and others (1981a), Robson and others (1981b), Robson (1987), Crifasi (1992), Raynolds and others (2001), Raynolds (2002, 2004), and Paschke (2011).

\section{Study Methods}

This section describes the methods used to identify target areas in Elbert County, which were used to select study wells, complete a Global Navigation Satellite System (GNSS) survey of well land-surface elevations, make and process groundwater-level measurements, access data, analyze trends in groundwater-level elevations throughout the county, and derive potentiometric-surface and difference maps from static groundwater-level measurements.

\section{Identifying Target Areas and Well Selection}

Areas within Elbert County were targeted for routine groundwater-level measurements using a combination of groundwater model statistics, groundwater model pumping scenarios, well density, and property access. The USGS MODFLOW-2000 groundwater model of the Denver Basin aquifer system from Paschke (2011) was used to determine areas within the county where groundwater-level observations could improve future model parameter calibration and decrease model uncertainty. A model analysis tool, OPR-PPR, combines Parameter-Prediction (PPR) statistics and Observation-Prediction (OPR) statistics to evaluate model uncertainty and its relation to observed data (Hill and Tiedeman, 2007; Tonkin and others, 2007). A statistic output from OPR-PRR, referred to by Tonkin and others (2007) as the OPA statistic, is defined as the percent change in parameter standard deviation caused by omitting or adding one or more observations. The OPA statistics for several pilot observation points in the Arapahoe aquifer in the Denver Basin model were combined into a single statistic called the Combined Observation-Parameter statistic (Paschke, 2011). Areas with a high statistic correspond to areas where the addition of observations could be used to decrease parameter uncertainty in the Arapahoe aquifer in future model calibration. The Combined Observation-Parameter statistic from Paschke (2011) identified areas in the north-central and northwestern parts of the county, indicated in figure 4 by dark grey shading, where additional observations could be beneficial if wells completed in the Arapahoe aquifer are accessible.

Future water-use scenarios presented in Paschke (2011) also were used to identify areas (target areas) where drawdown from pumping may affect groundwater levels. Water-use scenarios from 2003 through 2053 were simulated with the Denver Basin aquifer system model, and estimated drawdown maps, by aquifer, for the basin were created. Areas with a predicted drawdown greater than or equal to $100 \mathrm{ft}$ were identified in the western half of the county, predominantly along the northwestern border (fig. 4). Although these predictions are not definitive, they can guide monitoring efforts by identifying areas where future problems could arise. Areas of predicted drawdown greater than or equal to $100 \mathrm{ft}$ in the upper and lower Dawson aquifers fall within the Northwest and Elizabeth-Kiowa Planning Areas identified by the ECBOCC as focus areas for water-supply study and planning (Forsgren Associates Inc., 2018).

After target areas were identified, existing wells within the county were mapped by aquifer of completion using data from the CDWR well permit database (Colorado Department of Natural Resources, 2018). The density of wells was used to identify areas where monitoring could be conducted (fig. 4). Property owners with domestic supply wells in target areas were contacted through door-to-door solicitation. The primary focus was aimed at selecting wells in the upper Dawson, lower Dawson, and Denver aquifers, based on discussions with the ECBOCC. Several wells completed in the Arapahoe and Laramie-Fox Hills aquifers but outside target areas also were included to assess groundwater levels countywide. Forty-two domestic supply wells were initially selected for the network, including 11 in the upper Dawson aquifer, 10 in the lower 

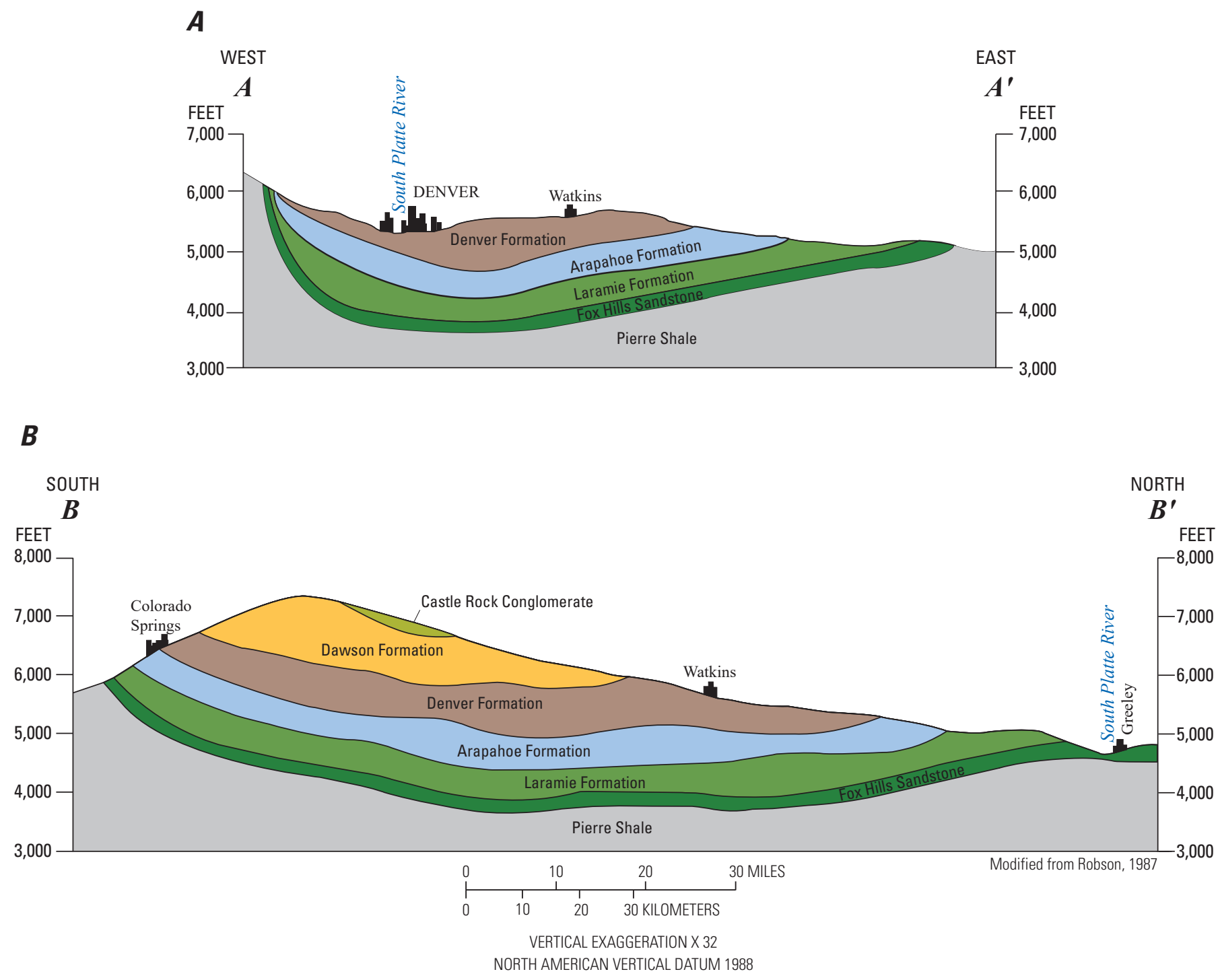

Figure 3. Generalized geologic cross sections $A, A-A^{\prime}$, west to east, and $B, B-B^{\prime}$, south to north, through the Denver Basin aquifer system. See figure 1 for cross section locations and table 1 for bedrock aquifer descriptions within each geologic unit.

Dawson aquifer, 7 in the Denver aquifer, 9 in the Arapahoe aquifer, and 5 in the Laramie-Fox Hills aquifer (table 2, fig. 2). Six of these wells were instrumented with a pressure transducer and data logger: 2 in the upper Dawson aquifer, 2 in the lower Dawson aquifer, 1 in the Denver aquifer, and 1 in the Laramie-Fox Hills aquifer. Instrumented wells were chosen on the basis of location, well access, and willingness of well owner to allow instrumentation.

\section{Geodetic Survey of Wells}

A measuring point (MP) was established on the casing of each well as a consistent point from which to make measurements (appendix fig. 1.1). The height of each MP above the land surface was manually measured. The elevation of each well's MP was determined using a real-time kinematic (RTK)-GNSS, following the methods described in Rydlund 
Table 1. Physical characteristics of bedrock aquifers in the Denver Basin aquifer system.

[See figure 1 for extent and location of aquifers and exent and location of Elbert County. mi², square mile; ft, foot; UDAW, upper Dawson aquifer; LDAW, lower Dawson aquifer; DENV, Denver aquifer; ARAP, Arapahoe aquifer; LARA, Laramie-Fox Hills aquifer; N/A, not applicable]

\begin{tabular}{|c|c|c|c|c|c|c|c|c|c|}
\hline $\begin{array}{l}\text { Bedrock } \\
\text { aquifer }\end{array}$ & Aquifer & $\begin{array}{l}\text { Total } \\
\text { surface } \\
\text { area } \\
\left(\mathrm{mi}^{2}\right)\end{array}$ & $\begin{array}{l}\text { Area } \\
\text { within } \\
\text { Elbert } \\
\text { County } \\
\left(\mathrm{mi}^{2}\right)\end{array}$ & $\begin{array}{l}\text { Minimum } \\
\text { thickness } \\
\quad(\mathrm{ft})\end{array}$ & $\begin{array}{l}\text { Maximum } \\
\text { thickness } \\
\quad(\mathrm{ft})\end{array}$ & $\begin{array}{l}\text { Average } \\
\text { water- } \\
\text { yielding } \\
\text { thickness } \\
\text { (ft) }\end{array}$ & Composition & Age & $\begin{array}{l}\text { Top } \\
\text { confining } \\
\text { layers }\end{array}$ \\
\hline Upper Dawson & UDAW & 600 & 302 & \multirow{2}{*}{100} & \multirow{2}{*}{1,100} & \multirow{2}{*}{$100-400$} & \multirow{2}{*}{$\begin{array}{l}\text { Dawson Formation: interbedded fluvial } \\
\text { conglomerate, sandstone, siltstone, } \\
\text { shale }\end{array}$} & \multirow{2}{*}{ Tertiary } & N/A - unconfined \\
\hline Lower Dawson & LDAW & 1,400 & 423 & & & & & & clay and shale \\
\hline Denver & DENV & 3,200 & 830 & 600 & 1,200 & $100-300$ & $\begin{array}{l}\text { Denver Formation: interbedded shale, } \\
\text { claystone, siltstone, sandstone, coal, } \\
\text { and volcanic ash and rocks }\end{array}$ & $\begin{array}{l}\text { Late Cretaceous to } \\
\text { Early Tertiary }\end{array}$ & $\begin{array}{l}\text { Heterogeneous clay- } \\
\text { stone and shale }\end{array}$ \\
\hline Arapahoe & ARAP & 4,700 & 1,160 & 400 & 700 & $200-300$ & $\begin{array}{l}\text { Arapahoe Formation: interbedded con- } \\
\text { glomerate, sandstone, siltstone, shale }\end{array}$ & Late Cretaceous & $\begin{array}{l}\text { Upper Arapahoe } \\
\text { Formation fine- } \\
\text { grained deposits }\end{array}$ \\
\hline \multirow{2}{*}{$\begin{array}{l}\text { Laramie-Fox } \\
\text { Hills }\end{array}$} & \multirow{2}{*}{ LARA } & \multirow{2}{*}{7,000} & \multirow{2}{*}{1,538} & \multirow{2}{*}{10} & \multirow{2}{*}{400} & \multirow{2}{*}{150} & $\begin{array}{l}\text { Laramie Formation: very fine- to } \\
\text { medium-grained sandstone with } \\
\text { interstitial silt and clay }\end{array}$ & \multirow{2}{*}{ Late Cretaceous } & \multirow{2}{*}{$\begin{array}{l}\text { Upper Laramie } \\
\text { Formation gray to } \\
\text { black shale, coal } \\
\text { seams, siltstone, } \\
\text { sandstone }\end{array}$} \\
\hline & & & & & & & $\begin{array}{l}\text { Fox Hills Sandstone Formation: very } \\
\text { fine grained silty sandstone and shaly } \\
\text { siltstone with interbedded shale }\end{array}$ & & \\
\hline
\end{tabular}

Sources:

Romero, 1976

Kirkham and Ladwig, 1979

Schneider, 1980

Robson and others, 1981a

Robson and others, 1981b

Robson, 1987

Crifasi, 1992

Raynolds and others, 2001

Raynolds, 2002

Raynolds, 2004

Paschke, 2011 


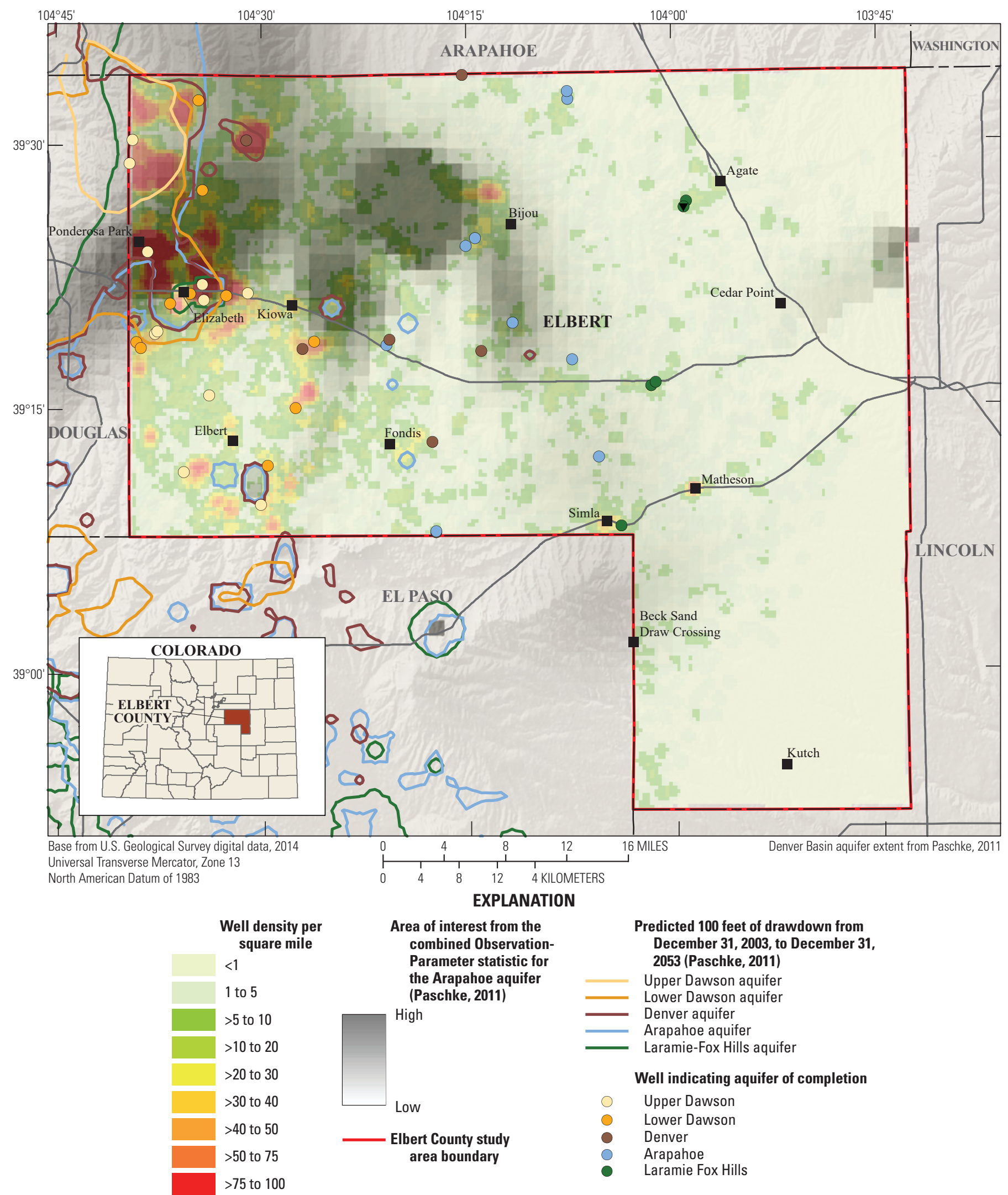

Figure 4. Location of wells completed in Denver Basin bedrock aquifers, predicted 100-foot drawdown, well density, and areas of interest in the Arapahoe aquifer, for groundwater-level monitoring in Elbert County, Colorado. 
and Densmore (2012). The MPs at 39 wells were surveyed in July or August 2017, and the MPs at 3 wells were surveyed in May 2018. Surveys were completed with a RTK-GNSS base station equipped with a radio to communicate with a RTK-GNSS rover equipped with a GNSS receiver and a data logger. The rover was fixed to a survey rod with known height and a bubble level. Each base station was located at a fixed position, and a static survey was performed for a period of 2-4 hours. The rover was used to identify the coordinates and elevation of each MP while communicating with a base station. At most wells, the MP was surveyed twice to check measurement accuracy. Location data from the base station static surveys were submitted to the National Geodetic Survey's Online Positioning User Service (OPUS) web portal (National Geodetic Survey, 2018) for processing. The corrected base station data from the OPUS solution were used to correct all MP locations, which are projected in the Universal Transverse Mercator (UTM) Zone 13 North coordinate system; North American Datum of 1983 (NAD 83) horizontal datum; and North American Vertical Datum of 1988 (NAVD 88), Geoid 12B, ellipsoid World Geodetic System 1984 (WGS 84) vertical datum.

The land-surface datum (LSD) of each well was calculated by subtracting the well MP height from the MP elevation determined by the survey. Coordinates and elevation for each well are summarized in table 2. By computing the elevation of LSD for each well on a consistent coordinate system, horizontal datum, and vertical datum, groundwater levels can be accurately compared across Elbert County.

\section{Groundwater-Level Measurements and Groundwater-Level Elevations}

This section presents the methods used for making and processing the discrete and continuous groundwater-level measurements analyzed in this study. Groundwater levels are presented as depth to groundwater in ft. below LSD. Calculated groundwater-level elevations are presented in $\mathrm{ft}$ above NAVD 88.

\section{Discrete Groundwater-Level Measurements and Groundwater-Level Elevations}

Groundwater levels were routinely measured in 42 wells (table 2) during the study period, April 2015 to June 2018. Routine measurements were discontinued at one well, common name DENV 13 (table 2), in June 2016 and at well LARA 4 in October 2017 because of difficulties with well access. Routine measurements were suspended at well UDAW 18 from December 2016 to December 2017 during a change in property ownership. Manual measurements were made bi-monthly (February, April, June, August, October,
December) except for a few instances when well access was temporarily restricted. The procedures for making manual groundwater-level measurements are outlined in Cunningham and Schalk (2011), with the exception that a breakaway weight was not used because of concerns it could get entangled with pump wiring or piping.

For each measurement, a calibrated steel tape was lowered down the well to record the depth to groundwater from the well MP (appendix fig. 1.1). In instances where a steel tape could not be used (such as excessive moisture causing fouled readings, erratic levels due to pumping, difficulties with a well access port), a calibrated electrical tape was used (appendix fig. 1.1). In each instance, the depth to groundwater from the MP was recorded to the nearest $0.01 \mathrm{ft}$ and corrected for the height of the MP above LSD to give a final reading of measured depth to groundwater below LSD. To determine whether the groundwater level measured in the well was static and to follow USGS protocol as a quality-control measure, a second check measurement was made, typically 3-5 minutes after the first. Measurements that differed by $0.02 \mathrm{ft}$ or less were considered static and a reliable measurement.

When the check measurement did not agree with the original measurement, additional measurements were made until the reason for lack of agreement was determined, or results were shown to be reliably representative of field conditions. Subsequent measurements were taken to document the status of the groundwater level in the well. If consecutive measurements indicated a rising groundwater level (decreasing depth to groundwater), the well was considered to be recovering from recent pumping, and the highest groundwater level (smallest depth to groundwater) measured during the field visit was assigned a status of " $\mathrm{R}$," indicating recently pumped. Typical reasons for recently pumped wells include water use for lawn irrigation systems, washing machines, or flushing toilets. If consecutive measurements indicated a slowly decreasing groundwater level (increasing depth to groundwater), the well was considered to be affected by one or more wells pumping nearby from the same aquifer, and the highest groundwater level measured during the field visit was assigned a status of "S," indicating nearby pumping. Typical reasons for nearby pumping include agricultural operations or domestic use. If the pump in the well was cycling on and off, the measured depth to groundwater was usually erratic and did not follow a pattern. If the well owners were available, they were asked to temporarily suspend water use during the field visit so that an " $R$ " status could be obtained. If the pump could not be turned off, the highest groundwater level (smallest depth to groundwater) measured during the field visit was given the status of "P," indicating pumping. Static measurements, which were made 80 percent of the time, are ideal for assessing changes and trends in aquifer groundwater levels. 
[Site identification numbers in this table are hyperlinked to the data in NWIS Web http://dx.doi.org/10.5066/F7P55KJN. See figure 2 for well locations. Bold, indicates site instrumented with a pressure transducer. Underline, indicates sites with no pump in well. NAD 83, North American Datum of 1983; NAVD 88, North American Vertical Datum of 1988; ft, foot; bls, below land surface; "R", recently pumped; "S", nearby pumping; "P”, pumping; LSD, land-surface datum; UDAW, upper Dawson aquifer; LDAW, lower Dawson aquifer; DENV, Denver aquifer; ARAP, Arapahoe aquifer; LARA, LaramieFox Hills aquifer; ${ }^{\circ}$, degrees; ', minutes; “, seconds]

\begin{tabular}{|c|c|c|c|c|c|c|c|c|c|c|c|c|c|}
\hline $\begin{array}{c}\text { Site } \\
\text { identification } \\
\text { number }\end{array}$ & Aquifer & $\begin{array}{c}\text { Common } \\
\text { name }\end{array}$ & $\begin{array}{l}\text { Latitude } \\
\text { (NAD 83) } \\
\text { (degrees, } \\
\text { minutes, } \\
\text { seconds) }\end{array}$ & $\begin{array}{c}\text { Longitude } \\
\text { (NAD 83) } \\
\text { (degrees, } \\
\text { minutes, } \\
\text { seconds) }\end{array}$ & $\begin{array}{c}\text { Elevation of } \\
\text { LSD } \\
\text { (ft above } \\
\text { NAVD 88) }\end{array}$ & $\begin{array}{l}\text { Well } \\
\text { depth } \\
\text { (ft bls) }\end{array}$ & $\begin{array}{c}\text { Total } \\
\text { measure- } \\
\text { ments }\end{array}$ & $\begin{array}{c}\text { Number } \\
\text { of static } \\
\text { measure- } \\
\text { ments }\end{array}$ & $\begin{array}{l}\text { Number of } \\
\text { measure- } \\
\text { ments with } \\
\text { status “R" }\end{array}$ & $\begin{array}{l}\text { Number of } \\
\text { measure- } \\
\text { ments with } \\
\text { status "S" }\end{array}$ & $\begin{array}{l}\text { Number of } \\
\text { measure- } \\
\text { ments with } \\
\text { status "P" }\end{array}$ & $\begin{array}{c}\text { Average } \\
\text { depth to } \\
\text { water below } \\
\text { LSD (ft) }\end{array}$ & $\begin{array}{c}\text { Average } \\
\text { groundwater } \\
\text { elevation } \\
\text { (ft above } \\
\text { NAVD 88) }\end{array}$ \\
\hline 390935104301001 & UDAW & DAWMAS26 & $39^{\circ} 09^{\prime} 90935^{\prime \prime}$ & $-104^{\circ} 30^{\prime} 010.5^{\prime \prime}$ & $7,200.00$ & 500 & 18 & 12 & 5 & 0 & 1 & 348.84 & $6,851.16$ \\
\hline 391126104354701 & UDAW & UDAW 19 & $39^{\circ} 11^{\prime} 126.6^{\prime \prime}$ & $-104^{\circ} 35^{\prime} 547.8^{\prime \prime}$ & $7,118.00$ & 401 & 21 & 17 & 4 & 0 & 0 & 263.52 & $6,854.48$ \\
\hline 391545104335401 & UDAW & DAWMAS22 & $39^{\circ} 15^{\prime} 545.5^{\prime \prime}$ & $-104^{\circ} 33^{\prime} 354.6^{\prime \prime}$ & $6,835.00$ & 360 & 20 & 20 & 0 & 0 & 0 & 166.13 & $6,668.87$ \\
\hline 391915104375001 & UDAW & UDAW 18 & $39^{\circ} 19^{\prime} 28.24^{\prime \prime}$ & $-104^{\circ} 37^{\prime} 50.16^{\prime \prime}$ & $6,759.62$ & 340 & 10 & 9 & 1 & 0 & 0 & 163.05 & $6,596.57$ \\
\hline$\underline{391924104374101}$ & UDAW & UDAW 14 & $\underline{39^{\circ} 19^{\prime} 24.51^{\prime \prime}}$ & $-104^{\circ} 37^{\prime} 46.62^{\prime \prime}$ & $\underline{6,783.97}$ & $\underline{300}$ & $\underline{24}$ & $\underline{24}$ & $\underline{\mathbf{0}}$ & $\underline{\mathbf{0}}$ & $\underline{\mathbf{0}}$ & $\underline{184.44}$ & $\underline{6,599.53}$ \\
\hline 392130104341401 & UDAW & UDAW 17 & $39^{\circ} 21^{\prime} 29.54^{\prime \prime}$ & $-104^{\circ} 34^{\prime} 15.42^{\prime \prime}$ & $6,678.27$ & 270 & 20 & 12 & 8 & 0 & 0 & 193.13 & $6,485.14$ \\
\hline 392133104310201 & UDAW & UDAW 12 & $39^{\circ} 21^{\prime} 131.9^{\prime \prime}$ & $-104^{\circ} 31^{\prime} 06.46^{\prime \prime}$ & $6,613.45$ & 225 & 25 & 25 & $\mathbf{0}$ & $\mathbf{0}$ & $\mathbf{0}$ & 172.82 & $6,440.63$ \\
\hline 392203104342301 & UDAW & UDAW 16 & $39^{\circ} 22^{\prime} 03.34^{\prime \prime}$ & $-104^{\circ} 34^{\prime} 22.54^{\prime \prime}$ & $6,638.05$ & 312 & 20 & 20 & 0 & 0 & 0 & 184.41 & $6,453.64$ \\
\hline 392355104382001 & UDAW & UDAW 15 & $39^{\circ} 23^{\prime} 355.8^{\prime \prime}$ & $-104^{\circ} 38^{\prime} 820.9^{\prime \prime}$ & $6,585.00$ & 290 & 20 & 18 & 2 & 0 & 0 & 190.12 & $6,394.88$ \\
\hline 392856104393801 & UDAW & UDAW 13 & $39^{\circ} 28^{\prime} 57.89^{\prime \prime}$ & $-104^{\circ} 39^{\prime} 38.05^{\prime \prime}$ & $6,403.45$ & 300 & 20 & 19 & 1 & 0 & 0 & 167.72 & $6,235.73$ \\
\hline 393016104392601 & UDAW & UDAW 11 & $39^{\circ} 30^{\prime} 17.99^{\prime \prime}$ & $-104^{\circ} 39^{\prime} 26.73^{\prime \prime}$ & $6,276.70$ & 340 & 20 & 19 & 1 & 0 & 0 & 96.24 & $6,180.46$ \\
\hline 391148104294101 & LDAW & DAWMAS27 & $39^{\circ} 11^{\prime} 148.9^{\prime \prime}$ & $-104^{\circ} 29^{\prime} 941.7^{\prime \prime}$ & $6,960.00$ & 475 & 20 & 18 & 2 & 0 & 0 & 270.87 & $6,689.13$ \\
\hline 391502104273601 & LDAW & LDAW 16 & $39^{\circ} 15^{\prime} 502.2^{\prime \prime}$ & $-104^{\circ} 27^{\prime} 735.8^{\prime \prime}$ & $6,750.00$ & 441 & 20 & 6 & 13 & 1 & 0 & 149.67 & $6,600.33$ \\
\hline 391829104385301 & LDAW & LDAW 15 & $39^{\circ} 18^{\prime} 25.51^{\prime \prime}$ & $-104^{\circ} 38^{\prime} 49.21^{\prime \prime}$ & $6,754.82$ & 743 & 20 & 13 & 6 & 1 & 0 & 206.71 & $6,548.11$ \\
\hline 391848104261401 & LDAW & DAWMAS28 & $39^{\circ} 18^{\prime} 848.8^{\prime \prime}$ & $-104^{\circ} 26^{\prime} 614.9^{\prime \prime}$ & $6,740.00$ & 388 & 19 & 16 & 3 & 0 & 0 & 264.41 & $6,475.59$ \\
\hline 391852104391301 & LDAW & DAWMAS16 & $39^{\circ} 18^{\prime} 52.56^{\prime \prime}$ & $-104^{\circ} 39^{\prime} 12.96^{\prime \prime}$ & $6,798.32$ & 720 & 20 & 8 & 11 & 1 & 0 & 275.33 & $6,522.99$ \\
\hline 392058104364401 & LDAW & LDAW 12 & $39^{\circ} 20^{\prime} 58.84^{\prime \prime}$ & $-104^{\circ} 36^{\prime} 44.49^{\prime \prime}$ & $6,606.29$ & 540 & 22 & 21 & 1 & $\mathbf{0}$ & $\mathbf{0}$ & 177.69 & $6,428.60$ \\
\hline 392125104323701 & LDAW & LDAW 14 & $39^{\circ} 21^{\prime} 25.24^{\prime \prime}$ & $-104^{\circ} 32^{\prime} 38.44^{\prime \prime}$ & $6,599.92$ & 415 & 20 & 19 & 0 & 1 & 0 & 149.81 & $6,450.11$ \\
\hline 392131104351701 & LDAW & DAWMAS21 & $39^{\circ} 21^{\prime} 31.49^{\prime \prime}$ & $-104^{\circ} 35^{\prime} 17.53^{\prime \prime}$ & $6,513.63$ & 435 & 20 & 14 & 6 & 0 & 0 & 96.16 & $6,417.47$ \\
\hline 392724104341901 & LDAW & LDAW 13 & $39^{\circ} 27^{\prime} 727.1^{\prime \prime}$ & $-104^{\circ} 34^{\prime} 417.1^{\prime \prime}$ & $6,305.00$ & 440 & 20 & 18 & 2 & 0 & 0 & 129.82 & $6,175.18$ \\
\hline 393227104343401 & LDAW & DAWMAS19 & $39^{\circ} 32^{\prime} 27.27^{\prime \prime}$ & $-104^{\circ} 34^{\prime} 34.47^{\prime \prime}$ & $6,257.95$ & 320 & 25 & 24 & 1 & 0 & 0 & 211.98 & $6,045.97$ \\
\hline 390755104172501 & DENV & DENV 17 & $39^{\circ} 07^{\prime} 55.35^{\prime \prime}$ & $-104^{\circ} 17^{\prime} 25.48^{\prime \prime}$ & $6,440.23$ & 480 & 16 & 2 & 13 & 0 & 1 & 265.17 & $6,175.06$ \\
\hline$\underline{391257104173601}$ & DENV & DENV 16 & $\underline{39^{\circ} 12^{\prime} 58.39^{\prime \prime}}$ & $-104^{\circ} 17^{\prime} 38.35^{\prime \prime}$ & $\underline{6,298.93}$ & $\underline{140}$ & $\underline{20}$ & $\underline{20}$ & $\underline{0}$ & $\underline{0}$ & $\underline{0}$ & $\underline{82.18}$ & $\underline{6,216.75}$ \\
\hline 391811104140301 & DENV & DENV 15 & $39^{\circ} 18^{\prime} 825.5^{\prime \prime}$ & $-104^{\circ} 13^{\prime} 358.1^{\prime \prime}$ & $6,005.48$ & 280 & 20 & 15 & 5 & 0 & 0 & 140.74 & $5,864.74$ \\
\hline 391821104270601 & DENV & DENV 14 & $39^{\circ} 18^{\prime} 821.6^{\prime \prime}$ & $-104^{\circ} 27^{\prime} 706.4^{\prime \prime}$ & $6,644.00$ & 923 & 19 & 14 & 4 & 1 & 0 & 241.85 & $6,402.15$ \\
\hline 391851104204501 & DENV & DENMAS05 & $39^{\circ} 18^{\prime} 851.9^{\prime \prime}$ & $-104^{\circ} 20^{\prime} 045.5^{\prime \prime}$ & $6,080.00$ & 545 & 20 & 15 & 2 & 0 & 3 & 258.05 & $5,821.95$ \\
\hline 393012104310701 & DENV & DENV 13 & $39^{\circ} 30^{\prime} 012.5^{\prime \prime}$ & $-104^{\circ} 31^{\prime} 107.3^{\prime \prime}$ & $6,245.00$ & 534 & 8 & 7 & 1 & 0 & 0 & 340.53 & $5,904.47$ \\
\hline$\underline{393350104151701}$ & $\underline{\text { DENV }}$ & DENV 12 & $\underline{39^{\circ} 33^{\prime} 51.99^{\prime \prime}}$ & $-104^{\circ} 15^{\prime} 17.07^{\prime \prime}$ & $\underline{\mathbf{5 , 5 8 7 . 6 1}}$ & $\underline{161}$ & $\underline{23}$ & $\underline{23}$ & $\underline{\mathbf{0}}$ & $\underline{\mathbf{0}}$ & $\underline{\mathbf{0}}$ & $\underline{114.10}$ & $\underline{5,473.51}$ \\
\hline 390800104172601 & ARAP & ARAP 8 & $39^{\circ} 08^{\prime} 00.68^{\prime \prime}$ & $-104^{\circ} 17^{\prime} 23.86^{\prime \prime}$ & $6,426.37$ & 730 & 20 & 9 & 8 & 0 & 3 & 379.13 & $6,047.24$ \\
\hline
\end{tabular}


Table 2. Well identification and location information, and a summary of discrete groundwater-level measurements from April 2015 to June 2018 in Elbert County, Colorado.-Continued

[Site identification numbers in this table are hyperlinked to the data in NWIS Web http://dx.doi.org/10.5066/F7P55KJN. See figure 2 for well locations. Bold, indicates site instrumented with a pressure transducer. Underline, indicates sites with no pump in well. NAD 83, North American Datum of 1983; NAVD 88, North American Vertical Datum of 1988; ft, foot; bls, below land surface; "R", recently pumped; "S", nearby pumping; "P”, pumping; LSD, land-surface datum; UDAW, upper Dawson aquifer; LDAW, lower Dawson aquifer; DENV, Denver aquifer; ARAP, Arapahoe aquifer; LARA, LaramieFox Hills aquifer; ', degrees; ', minutes; “, seconds]

\begin{tabular}{|c|c|c|c|c|c|c|c|c|c|c|c|c|c|}
\hline $\begin{array}{c}\text { Site } \\
\text { identification } \\
\text { number }\end{array}$ & Aquifer & $\begin{array}{l}\text { Common } \\
\text { name }\end{array}$ & $\begin{array}{l}\text { Latitude } \\
\text { (NAD 83) } \\
\text { (degrees, } \\
\text { minutes, } \\
\text { seconds) }\end{array}$ & $\begin{array}{l}\text { Longitude } \\
\text { (NAD 83) } \\
\text { (degrees, } \\
\text { minutes, } \\
\text { seconds) }\end{array}$ & $\begin{array}{c}\text { Elevation of } \\
\text { LSD } \\
\text { (ft above } \\
\text { NAVD 88) }\end{array}$ & $\begin{array}{c}\text { Well } \\
\text { depth } \\
\text { (ft bls) }\end{array}$ & $\begin{array}{c}\text { Total } \\
\text { measure- } \\
\text { ments }\end{array}$ & $\begin{array}{c}\text { Number } \\
\text { of static } \\
\text { measure- } \\
\text { ments }\end{array}$ & $\begin{array}{l}\text { Number of } \\
\text { measure- } \\
\text { ments with } \\
\text { status "R" }\end{array}$ & $\begin{array}{l}\text { Number of } \\
\text { measure- } \\
\text { ments with } \\
\text { status "S" }\end{array}$ & $\begin{array}{l}\text { Number of } \\
\text { measure- } \\
\text { ments with } \\
\text { status "P" }\end{array}$ & $\begin{array}{c}\text { Average } \\
\text { depth to } \\
\text { water below } \\
\text { LSD (ft) }\end{array}$ & $\begin{array}{c}\text { Average } \\
\text { groundwater } \\
\text { elevation } \\
\text { (ft above } \\
\text { NAVD 88) }\end{array}$ \\
\hline 391208104053301 & ARAP & ARAP 7 & $39^{\circ} 12^{\prime} 09.62^{\prime \prime}$ & $-104^{\circ} 05^{\prime} 33.73^{\prime \prime}$ & $6,131.66$ & 320 & 20 & 17 & 3 & 0 & 0 & 147.28 & $5,984.38$ \\
\hline 391740104072401 & ARAP & ARAPMAS27 & $39^{\circ} 17^{\prime} 40.17^{\prime \prime}$ & $-104^{\circ} 07^{\prime} 24.14^{\prime \prime}$ & $5,867.46$ & 130 & 18 & 15 & 3 & 0 & 0 & 56.40 & $5,811.06$ \\
\hline 391834104205601 & ARAP & ARAPMAS22 & $39^{\circ} 18^{\prime} 834.6^{\prime \prime}$ & $-104^{\circ} 20^{\prime} 42056^{\prime \prime}$ & $6,140.00$ & 832 & 20 & 16 & 4 & 0 & 0 & 311.19 & $5,828.81$ \\
\hline 391946104114501 & ARAP & ARAP 6 & $39^{\circ} 19^{\prime} 47.26^{\prime \prime}$ & $-104^{\circ} 11^{\prime \prime \prime} 45.41^{\prime \prime}$ & $6,159.61$ & 580 & 20 & 19 & 1 & 0 & 0 & 292.72 & $5,866.89$ \\
\hline 392400104150601 & ARAP & ARAPMAS28 & $39^{\circ} 24^{\prime} 00.71^{\prime \prime}$ & $-104^{\circ} 15^{\prime} 06.36^{\prime \prime}$ & $5,921.21$ & 434 & 20 & 19 & 1 & 0 & 0 & 206.07 & $5,715.14$ \\
\hline 392434104142701 & ARAP & ARAP 5 & $39^{\circ} 24^{\prime} 37.75^{\prime \prime}$ & $-104^{\circ} 14^{\prime} 24.48^{\prime \prime}$ & $6,082.67$ & 425 & 20 & 20 & 0 & 0 & 0 & 334.97 & $5,747.70$ \\
\hline 393225104073601 & ARAP & ARAP 4 & $39^{\circ} 32^{\prime} 24.71^{\prime \prime}$ & $-104^{\circ} 07^{\prime} 39.92^{\prime \prime}$ & $5,473.72$ & 287 & 20 & 13 & 7 & 0 & 0 & 51.48 & $5,422.24$ \\
\hline 393251104073701 & ARAP & ARAP 3 & $39^{\circ} 32^{\prime} 52.91^{\prime \prime}$ & $-104^{\circ} 07^{\prime} 37.25^{\prime \prime}$ & $5,487.00$ & 360 & 20 & 14 & 6 & 0 & 0 & 109.95 & $5,377.05$ \\
\hline 390817104040301 & LARA & LARA 7 & $39^{\circ} 08^{\prime} 12.65^{\prime \prime}$ & $-104^{\circ} 04^{\prime} 04.33^{\prime \prime}$ & $5,937.18$ & 438 & 20 & 12 & 6 & 0 & 2 & 137.84 & $5,799.35$ \\
\hline 391609104014001 & LARA & LARA 6 & $39^{\circ} 16^{\prime} 12.29^{\prime \prime}$ & $-104^{\circ} 01^{\prime} 26.33^{\prime \prime}$ & $5,753.80$ & 340 & 20 & 19 & 1 & 0 & 0 & 145.55 & $5,608.25$ \\
\hline 391621104012001 & LARA & LARA 5 & $39^{\circ} 16^{\prime} 20.73^{\prime \prime}$ & $-104^{\circ} 01^{\prime} 32.11^{\prime \prime}$ & $5,746.10$ & 400 & 20 & 19 & 1 & 0 & 0 & 141.03 & $5,605.07$ \\
\hline 392616103591001 & LARA & LARA 3 & $39^{\circ} 26^{\prime} 17.16^{\prime \prime}$ & $-103^{\circ} 59^{\prime} 11.98^{\prime \prime}$ & $5,495.98$ & 340 & 22 & 22 & $\mathbf{0}$ & $\mathbf{0}$ & $\mathbf{0}$ & 84.95 & $5,411.03$ \\
\hline 392635103590001 & LARA & LARA 4 & $39^{\circ} 26^{\prime} 38.58^{\prime \prime}$ & $-103^{\circ} 58^{\prime} 59.23^{\prime \prime}$ & $5,480.04$ & 221 & 16 & 15 & 1 & 0 & 0 & 79.76 & $5,400.28$ \\
\hline
\end{tabular}


In this report, groundwater-level elevation is calculated from groundwater level below LSD according to the following equation:

$$
\text { Groundwater-level elevation }=L S D-\text { Water level below } L S D
$$

where

Groundwater-level elevation is groundwater-level elevation, in $\mathrm{ft}$ above NAVD 88;

LSD

Water level below $L S D$

is land-surface datum, in $\mathrm{ft}$ above NAVD 88 ; and

is measured depth, in $\mathrm{ft}$, to groundwater below land-surface

datum.

\section{Continuous Groundwater-Level Measurements and Groundwater-Level Elevations}

Six of the 42 wells in the monitoring network were instrumented with a pressure transducer containing an internal data logger (table 2, fig. 2). The pressure transducers are vented and rated for a 69-ft range in freshwater elevation, with a manufacturer accuracy of \pm 0.05 percent at 15 degrees Celsius (In-Situ Inc., 2017). The transducers are suspended in the well on a vented communication cable that allows the user to download data from the instrument while the transducer remains in place. The internal data logger was programmed to record depth to groundwater below LSD every hour, based on a static manual groundwaterlevel measurement at the time the transducer was deployed, following the methods described in Cunningham and Schalk (2011).

Each transducer was downloaded and serviced during each bi-monthly site visit. At the time of each manual groundwater-level measurement, a concurrent instantaneous transducer groundwater-level measurement was recorded. In instances where the transducer measurement had drifted greater than $0.10 \mathrm{ft}$ from the concurrent discrete groundwater-level measurement, the data logger was reset to match the discrete value for depth to groundwater. The continuous groundwater-level records were corrected to discrete groundwater-level measurements to account for instrument drift. Processing of the continuous groundwater-level measurements followed USGS guidelines (Freeman and others, 2004). Like discrete measurements, a continuous hourly record of groundwater-level elevation was calculated using equation 1. Daily values of groundwater-level elevations (medians, minimums, and maximums) were derived from the hourly groundwater-level elevation values.

\section{Accessing Data}

All discrete and continuous groundwater levels summarized in this report are publicly available through the USGS National Water Information System (NWIS) Web Interface at http://dx.doi.org/10.5066/F7P55KJN. The NWIS website provides an interface for accessing site information and data collected by the USGS and is regularly updated to reflect the most current data. Users of the interface can retrieve USGS data by category, region, site number, or many other criteria and produce tables and graphs for web viewing or export. In digital copies of this publication, site identification numbers in table 2 are hyperlinked to each well's NWIS web page. Data accessible from NWIS can be downloaded in R statistical software with the USGS dataRetrieval R package (Hirsch and De Cicco, 2015).

\section{Groundwater-Level Summary, Groundwater-Level Elevation Trend Analysis, and Mapping}

After measurement of, and corrections to, discrete and continuous groundwater levels, all groundwater levels used in this report went through an internal review and an independent approval process. In this report, minimum and maximum groundwater-level elevations are summarized by calendar year, and the relative change in groundwater-level elevation is 
computed as the difference between the April 2015 and April 2018 measurements. The difference in groundwater-level elevation from April 2015 to April 2018 was chosen so difference values would be comparable to those reported in Flor (2017), where differences were computed from measurements typically made in April and May of selected years. At wells with continuous hourly groundwater-level elevations, a dataset of daily maximum groundwaterlevel elevations was derived from the maximum hourly groundwater-level elevation measured each day. The daily maximum groundwater-level elevation values tend to represent periods of the day when pumping is not occurring at the well or in nearby wells and has not occurred recently. Median and minimum daily groundwater-level elevations derived from hourly measurements may have been affected by periods of pumping in most cases and thus may not reflect static conditions. The daily maximum groundwater-level elevations were used for reporting and trend analysis on continuous data.

Trends in groundwater-level elevations throughout Elbert County were determined by testing discrete and continuous groundwater-level elevations through time. A linear regression (eq. 2) and a linear regression with seasonality terms (eq. 3) (Helsel and Hirsch, 2002) for every well in the network were modeled using the R statistical program and the R Stats Package (R Core Team, 2018).

$$
Y=\beta_{0}+\left(\beta_{1} \times T\right)
$$

where

$$
\begin{array}{ll}
Y & \text { is groundwater-level elevation, in } \mathrm{ft} \text { above NAVD 88; } \\
T & \text { is time, in decimal years; } \\
\beta_{0} & \text { is intercept, in } \mathrm{ft} \text { above NAVD } 88 ; \text { and } \\
\beta_{1} & \text { is slope coefficient of T, in } \mathrm{ft} / \text { year. }
\end{array}
$$

$$
Y=\beta_{0}+\left(\beta_{1} \times T\right)+\left(\beta_{2} \times \sin (2 \pi T)\right)+\left(\beta_{3} \times \cos (2 \pi T)\right)
$$

where

$$
\begin{array}{ll}
Y & \text { is groundwater-level elevation, in } \mathrm{ft} \text { above NAVD 88; } \\
T & \text { is time, in decimal years; } \\
\beta_{0} & \text { is intercept, in } \mathrm{ft} \text { above NAVD } 88 ; \\
\beta_{1} & \text { is slope coefficient of T, in } \mathrm{ft} / \text { year; } \\
\beta_{2} & \text { is coefficient of seasonal sine term, years; and } \\
\beta_{3} & \text { is coefficient of seasonal cosine term, in years. }
\end{array}
$$

The slope coefficient of time $\left(\beta_{1}\right)$ is herein referred to as the trend in groundwater-level elevation. Only static groundwater levels were used for trend tests on discrete groundwaterlevel elevations to remove the effect of pumping on trends. A trend test was considered statistically significant when the probability value ( $p$-value) of $\beta_{1}$ was less than or equal to 0.10 and when the coefficient of determination $\left(\mathrm{R}^{2}\right)$ was greater than or equal to 0.40 . A negative trend indicates generally declining groundwater-level elevations in the well through time, and a positive trend indicates generally rising groundwater-level elevations in the well through time. If both trend tests were significant, the test with the highest $\mathrm{R}^{2}$ was reported. Trends in groundwater-level elevations through time (ft/year), based on significant trend tests, are summarized by well and by aquifer in this report.

Potentiometric-surface maps show the hydraulic head distribution of an area with contour lines of equal hydraulic head. Groundwater flow is from areas of high hydraulic head to areas of low hydraulic head; flow direction is perpendicular to the contours. The Ordinary Kriging (default options) and Contour functions in ArcMap Spatial Analyst Toolbox (Esri Inc., 1999-2016) were used to derive the hydraulic head distribution from static measurements in the county. Differences in the hydraulic head distribution from two or more points in time can highlight areas where groundwater-level elevations are rising or declining. Hydraulic head distributions were derived from static measurements in the upper Dawson and lower Dawson aquifers for April 2015 and April 2018. 


\section{Groundwater-Level Elevations in the Denver Basin Bedrock Aquifers of Elbert County}

During April 2015 to June 2018, more than 800 discrete and 5,800 continuous groundwater-level measurements were made in the Elbert County groundwater network. Hydrographs showing groundwater-level elevation through time for each well in the network are provided in appendix 2.

\section{Discrete Groundwater-Level Elevation Summary and Trends}

Groundwater levels in each of the Denver Basin bedrock aquifers varied both temporally and spatially. In general, groundwater-level elevations were lowest during summer and fall and recovered to higher elevations in winter and spring (table 3, appendix 2). Some wells exhibited strong seasonal fluctuations of several feet (for example, well UDAW 14, appendix fig. 2.22), whereas others show minimal seasonality with fluctuations less than $1 \mathrm{ft}$ between measurements (for example, well DENV 16, appendix fig. 2.8). Seasonal variations are caused by natural processes, including precipitation and evapotranspiration in aquifer zones connected to the land surface and timing of aquifer recharge in confined aquifer zones (Paschke, 2011). Human activities, such as increased irrigation for agriculture (during the growing season) and domestic pumping (for lawns), can also affect seasonal variations. Relative changes in discrete groundwater-level elevations between the April 2015 and April 2018 measurements are presented in table 3 . The 25 wells that exhibited a negative change represent a decline in groundwater-level elevation between the two measurements, whereas the 13 wells that exhibited a positive change represent a rise in groundwaterlevel elevation between the two measurements; 1 well exhibited no change. The largest groundwater-level elevation declines, based on static measurements, occurred in the lower Dawson and Arapahoe aquifers. The largest groundwater-level elevation rises, based on static measurements, occurred in the upper Dawson aquifer. All aquifers contained at least one well where the groundwater-level elevation rose and one well where groundwater-level elevation declined from April 2015 to April 2018. It is important to note that relative changes do not represent trends in groundwater-level elevation at a given well over the study period.

Of the 42 wells monitored in the study period, 20 exhibited statistically significant trends in groundwater-level elevations, based on the linear regression test (table 4, appendix table 3.1). Only wells with significant trends are summarized in table 4. Some wells had insufficient static measurements to ascertain whether a significant trend existed. Continued monitoring and more static measurements at the wells where trends were not significant may increase the likelihood of significant trend occurrence at those wells in the future. All aquifers except the upper Dawson had more wells with significant negative trends in groundwater-level elevations than wells with significant positive trends; however, at least one well within the upper Dawson, lower Dawson, and Arapahoe aquifers had a significant positive trend. For all aquifers except the upper Dawson, the average trend in groundwater-level elevation was negative, indicating generally declining groundwater-level elevations in those aquifers (table 4). The upper Dawson aquifer had 2 wells with negative trends and 3 wells with positive trends, with an average trend of $0.03 \mathrm{ft} /$ year, a maximum positive trend of $0.28 \mathrm{ft} /$ year, and a maximum negative trend of $-0.26 \mathrm{ft} /$ year. The lower Dawson aquifer had negative trends at 5 wells and a positive trend at only 1 well at the eastern edge of the aquifer's extent. The average trend in the lower Dawson aquifer was $-1.96 \mathrm{ft} /$ year, with one positive trend of $0.13 \mathrm{ft} /$ year and a maximum negative trend of $-4.91 \mathrm{ft} /$ year. The Denver aquifer had only one well with a significant trend of $-1.04 \mathrm{ft} /$ year. The Arapahoe aquifer had 4 wells with negative trends and 1 well with a positive trend. The average trend in the Arapahoe aquifer was $-0.46 \mathrm{ft} /$ year; the Arapahoe aquifer had a single positive trend of $0.38 \mathrm{ft} / \mathrm{year}$ and a maximum negative trend of $-0.94 \mathrm{ft} /$ year. The Laramie-Fox Hills aquifer had three wells with negative trends, an average trend of $-0.65 \mathrm{ft} /$ year, and a maximum negative trend of $-0.73 \mathrm{ft} /$ year. Except for the lower Dawson and Denver aquifers, all aquifers had significant trends that were within a narrow range of $\pm 1 \mathrm{ft} /$ year. The magnitude of negative groundwater-level elevation trends in the lower Dawson aquifer had a larger range than the other four aquifers, ranging from $-0.25 \mathrm{ft} /$ year to $-4.91 \mathrm{ft} /$ year, with 4 of the 5 negative trends in excess of $1 \mathrm{ft} /$ year.

Significant trends are relatively consistent for grouping and distribution throughout Elbert County, except for wells in the upper Dawson aquifer along the western border of the county, which have positive and negative trends (fig. 5). Wells that are in very close proximity and in the same aquifer generally agree in trend significance and direction (for example, LARA 5 and LARA 6, ARAP 3 and ARAP 4, DAWMAS2 1 and LDAW 12). A focus on the Elizabeth-Kiowa and Northwest Planning Areas (Forsgren Associates Inc., 2018) shows that the directionality of trends in the upper Dawson aquifer varies by location, whereas trends in the lower Dawson aquifer are consistently negative, especially LDAW 12 and DAWMAS21 in close proximity to the town of Elizabeth, Colo. (fig. 6). The locations of wells with negative trends in the lower Dawson aquifer are also central to the aquifer's extent.

\section{Continuous Groundwater-Level Elevation Summary and Trends}

Hydrographs of continuous groundwater-level elevations from the six wells containing pressure transducers were generally in agreement with discrete groundwater levels measured at the same well (appendix fig. 2.22, 2.24, 2.28, 2.33, 2.40, 2.42). Groundwater-level elevations were generally highest during winter and spring and lowest during summer and fall, except for DENV 12 (appendix fig. 2.42), which does not have 
Table 3. Summary of discrete groundwater-level elevations, April 2015 to June 2018, Elbert County, Colorado.

[See table 2 and figure 2 for well locations. R15, indicates April 2015 water level has status R (recently pumped). R18, indicates April 18 water level has status R (recently pumped). P15, indicates April 2015 water level has status P (pumping). P18, indicates April 2018 water level has status P (pumping). See "Study Methods" section for details. ft, foot; NAVD 88, North American Vertical Datum of 1988; UDAW, upper Dawson aquifer; LDAW, lower Dawson aquifer; DENV, Denver aquifer; ARAP, Arapahoe aquifer; LARA, Laramie-Fox Hills aquifer; --, no measurements; M, site missing either April 2015 or April 2018 measurement]

\begin{tabular}{|c|c|c|c|c|c|c|c|c|c|c|c|c|c|c|c|c|c|c|c|}
\hline \multirow[b]{2}{*}{$\begin{array}{c}\text { Site } \\
\text { identification } \\
\text { number }\end{array}$} & \multirow[b]{2}{*}{ Aquifer } & \multirow[b]{2}{*}{$\begin{array}{c}\text { Common } \\
\text { name }\end{array}$} & \multicolumn{4}{|c|}{2015} & \multicolumn{4}{|c|}{2016} & \multicolumn{4}{|c|}{2017} & \multicolumn{4}{|c|}{ January 2018 to June 2018} & \multirow[b]{2}{*}{$\begin{array}{r}\text { Relative } \\
\text { Change } \\
\text { April 201: } \\
\text { to April } \\
2018 \text { (ft) }\end{array}$} \\
\hline & & & $\begin{array}{l}\text { Maximum } \\
\text { ground- } \\
\text { water } \\
\text { elevation } \\
\text { (ft above } \\
\text { NAVD 88) }\end{array}$ & $\begin{array}{c}\text { Date of } \\
\text { maximum } \\
\text { measurement }\end{array}$ & $\begin{array}{l}\text { Minimum } \\
\text { ground- } \\
\text { water } \\
\text { elevation } \\
\text { (ft above } \\
\text { NAVD 88) }\end{array}$ & $\begin{array}{c}\text { Date of } \\
\text { minimum } \\
\text { measurement }\end{array}$ & $\begin{array}{l}\text { Maximum } \\
\text { ground- } \\
\text { water } \\
\text { elevation } \\
\text { (ft above } \\
\text { NAVD 88) }\end{array}$ & $\begin{array}{c}\text { Date of } \\
\text { maximum } \\
\text { measure- } \\
\text { ment }\end{array}$ & $\begin{array}{c}\text { Minimum } \\
\text { ground- } \\
\text { water } \\
\text { elevation } \\
\text { (ft above } \\
\text { NAVD 88) }\end{array}$ & $\begin{array}{c}\text { Date of } \\
\text { minimum } \\
\text { measure- } \\
\text { ment }\end{array}$ & $\begin{array}{c}\text { Maximum } \\
\text { ground- } \\
\text { water } \\
\text { elevation } \\
\text { (ft above } \\
\text { NAVD 88) }\end{array}$ & $\begin{array}{c}\text { Date of } \\
\text { maximum } \\
\text { measure- } \\
\text { ment }\end{array}$ & $\begin{array}{c}\text { Minimum } \\
\text { ground- } \\
\text { water } \\
\text { elevation } \\
\text { (ft above } \\
\text { NAVD 88) }\end{array}$ & $\begin{array}{c}\text { Date of } \\
\text { minimum } \\
\text { measure- } \\
\text { ment }\end{array}$ & 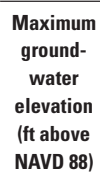 & $\begin{array}{c}\text { Date of } \\
\text { maximum } \\
\text { measure- } \\
\text { ment }\end{array}$ & $\begin{array}{c}\text { Minimum } \\
\text { ground- } \\
\text { water } \\
\text { elevation } \\
\text { (ft above } \\
\text { NAVD 88) }\end{array}$ & $\begin{array}{c}\text { Date of } \\
\text { minimum } \\
\text { measure- } \\
\text { ment }\end{array}$ & \\
\hline 390935104301001 & UDAW & DAWMAS26 & $6,851.60$ & 2015-12-11 & $6,850.95$ & 2015-10-05 & $6,851.27$ & 2016-02-15 & $6,850.38$ & 2016-08-12 & $6,851.40$ & $2017-02-03$ & $6,851.05$ & 2017-06-16 & $6,851.49$ & 2018-04-17 & $6,851.02$ & 2018-06-07 & -0.02 \\
\hline 91126104354701 & UDAW & UDAW 19 & $6,854.73$ & 2015-12-11 & $6,854.05$ & 2015-06-15 & $6,854.68$ & 2016-12-08 & $6,854.08$ & 2016-08-12 & $6,854.89$ & 2017-12-05 & $6,854.29$ & 2017-08-04 & $6,855.04$ & 2018-04-17 & $6,854.30$ & 2018-06-07 & 0.85 \\
\hline 91545104335401 & UDAW & DAWMAS22 & $6,668.98$ & $\begin{array}{l}2015-06-11 \\
2015-12-11\end{array}$ & $6,668.07$ & $2015-08$ & ,669.13 & 16-06-03 & $6,668.44$ & $2016-10$ & $6,669.42$ & 2017-04-13 & $6,668.52$ & 2017-08-04 & $6,669.42$ & $2018-0$ & $6,668.38$ & $2018-c$ & 0.47 \\
\hline 91915104375001 & UDAW & UDAW 18 & $6,597.95$ & 2015-12-14 & $6,594.23$ & 2015-10-05 & $6,598.00$ & 2016-06-06 & $6,594.83$ & 2016-08-10 & -- & -- & -- & -- & $6,598.16$ & $2018-02-14$ & $6,594.73$ & $2018-06-08$ & M \\
\hline 391924104374101 & UDAW & UDAW 14 & $6,600.57$ & 2015-06-11 & $6,597.50$ & 2015-10-05 & $6,600.63$ & 2016-02-19 & $6,597.77$ & 2016-08-10 & $6,600.84$ & 2017-02-01 & $6,598.18$ & $2017-06-26$ & $6,600.63$ & 2018-02-14 & $6,598.03$ & 2018-06-08 & -0.15 \\
\hline 92130104341401 & UDAW & UDAW 17 & $6,486.41$ & 2015-04-09 & $6,482.77$ & 2015-10-08 & $6,486.31$ & 2016-04-04 & $6,483.26$ & 2016-08-10 & $6,486.23$ & 2017-02-06 & $6,483.01$ & $2017-06-26$ & $6,486.32$ & 2018-04-12 & $6,482.92$ & 2018-06-08 & -0.09 \\
\hline 92133104310201 & UDAW & UDAW 12 & $6,440.41$ & $\begin{array}{l}2015-06-08 \\
2015-10-02\end{array}$ & $6,440.11$ & $2015-08$ & ,440.85 & $16-12-05$ & $6,440.25$ & $2016-10$ & $6,441.06$ & 2017-03-31 & $6,440.31$ & 03 & 441.41 & 201 & $6,440.91$ & $2018-02-13$ & 1.27 \\
\hline 92203104342301 & UDAW & UDAW 16 & $6,454.73$ & 2015-12-03 & $6,448.77$ & 2015-10-08 & $6,456.31$ & 2016-12-05 & $6,447.32$ & 2016-08-10 & $6,457.70$ & 2017-02-06 & $6,449.86$ & $2017-06-26$ & $6,457.69$ & 2018-04-12 & $6,450.08$ & 2018-06-06 & 3.62 \\
\hline 392355104382001 & UDAW & UDAW 15 & $6,396.90$ & 2015-04-10 & $6,393.50$ & 2015-10-08 & $6,395.94$ & 2016-02-19 & $6,392.11$ & 2016-08-10 & $6,396.25$ & 2017-04-03 & $6,393.73$ & $2017-06-26$ & $6,396.15$ & 2018-04-19 & $6,394.00$ & 2018-06-08 & -0.75 \\
\hline 92856104393801 & UDAW & UDAW 13 & $6,239.55$ & 2015-04-10 & $6,230.64$ & 2015-10-08 & $6,240.95$ & 2016-04-08 & $6,228.87$ & 2016-08-12 & $6,240.27$ & 2017-04-03 & $6,230.27$ & 2017-08-02 & $6,240.07$ & 2018-02-08 & $6,231.22$ & 2018-06-08 & 0.00 \\
\hline 393016104392601 & UDAW & UDAW 11 & $6,180.61$ & 2015-06-10 & $6,178.01$ & 2015-10-08 & $6,181.58$ & 2016-06-06 & $6,179.11$ & 2016-10-04 & $6,182.46$ & 2017-04-03 & $6,179.13$ & 2017-10-11 & $6,181.54$ & 2018-04-19 & $6,180.69$ & 2018-06-08 & 1.30 \\
\hline 91148104294101 & LDAW & DAWMAS27 & 6,689.54 & 2015-06-11 & $6,688.10$ & 2015-10-05 & $6,689.73$ & 2016-04-07 & $6,687.71$ & 2016-08-12 & $6,689.81$ & $2017-04-13$ & $6,688.54$ & 2017-08-04 & $6,689.82$ & 2018-04-17 & $6,689.35$ & 2018-06-07 & 0.41 \\
\hline 391502104273601 & LDAW & LDAW 16 & $6,602.18$ & 2015-04-02 & $6,597.77$ & 2015-08-07 & $6,601.97$ & 2016-04-07 & 6,597.22 & 2016-10-07 & $6,602.54$ & $2017-02-03$ & $6,599.48$ & 2017-04-13 & $6,602.50$ & 2018-02-14 & 6,599.34 & 2018-06-07 & $-1.89^{R 15}$ \\
\hline 91829104385301 & LDAW & LDAW 15 & $6,556.99$ & 2015-06-11 & $6,540.94$ & 2015-10-05 & $6,556.58$ & 2016-06-03 & $6,537.06$ & 2016-10-07 & $6,553.21$ & 2017-04-13 & $6,541.20$ & 2017-08-01 & $6,552.31$ & 2018-02-14 & $6,539.94$ & 2018-06-07 & -4.36 \\
\hline 391848104261401 & LDAW & DAWMAS28 & $6,477.84$ & 2015-12-11 & $6,474.17$ & 2015-08-07 & $6,476.20$ & 2016-12-08 & $6,472.59$ & 2016-08-12 & $6,475.93$ & $2017-04-13$ & $6,473.53$ & 2017-10-04 & $6,481.28$ & 2018-06-07 & $6,475.70$ & 2018-04-17 & -1.76 \\
\hline 391852104391301 & LDAW & DAWMAS16 & $6,536.19$ & 2015-04-02 & $6,506.02$ & 2015-10-05 & $6,537.70$ & 2016-04-07 & $6,507.86$ & 2016-10-07 & $6,531.82$ & 2017-02-03 & $6,508.96$ & 2017-10-04 & 6,533.54 & 2018-04-17 & $6,516.72$ & 2018-06-07 & $-2.65^{\mathrm{R} 18}$ \\
\hline 392058104364401 & LDAW & LDAW 12 & $6,445.36$ & 2015-04-02 & $6,417.96$ & 2015-10-05 & $6,441.14$ & 2016-04-08 & $6,416.47$ & 2016-08-09 & $6,437.49$ & 2017-04-03 & $6,410.56$ & 2017-10-04 & $6,435.82$ & 2018-04-17 & $6,417.71$ & 2018-06-08 & -9.54 \\
\hline 392125104323701 & LDAW & LDAW 14 & $6,451.32$ & 2015-04-09 & $6,447.35$ & 2015-10-01 & $6,452.27$ & 2016-06-02 & $6,447.11$ & 2016-08-09 & $6,452.67$ & 2017-04-03 & $6,446.60$ & 2017-08-03 & $6,451.72$ & 2018-02-13 & $6,447.54$ & 2018-06-04 & 0.22 \\
\hline 392131104351701 & LDAW & DAWMAS21 & $6,426.25$ & 2015-04-09 & $6,415.28$ & 2015-10-08 & $6,424.48$ & 2016-06-06 & $6,410.72$ & 2016-10-12 & $6,420.38$ & 2017-04-03 & $6,408.16$ & 2017-10-12 & $6,418.96$ & 2018-04-12 & $6,415.42$ & 2018-06-06 & -7.29 \\
\hline 392724104341901 & LDAW & LDAW 13 & $6,182.30$ & 2015-06-10 & $6,174.10$ & 2015-12-10 & $6,182.47$ & 2016-06-06 & $6,164.35$ & 2016-10-04 & $6,178.34$ & 2017-04-03 & $6,164.58$ & 2017-08-09 & $6,180.16$ & 2018-04-19 & $6,170.91$ & 2018-06-08 & -1.99 \\
\hline 393227104343401 & LDAW & DAWMAS19 & $6,046.81$ & 2015-12-14 & $6,045.89$ & 2015-10-08 & $6,046.41$ & 2016-12-05 & $6,045.79$ & 2016-08-11 & $6,046.20$ & 2017-04-03 & $6,045.04$ & $2017-12-13$ & $6,045.78$ & 2018-02-08 & $6,045.35$ & 2018-06-08 & -0.74 \\
\hline 390755104172501 & DENV & DENV 17 & $6,183.88$ & 2015-06-08 & $6,176.09$ & 2015-12-10 & $6,179.54$ & 2016-02-12 & $6,175.46$ & 2016-04-04 & $6,180.19$ & $2017-12-12$ & $6,168.95$ & 2017-06-15 & $6,161.15$ & 2018-06-06 & $6,160.27$ & 2018-02-13 & $-21.92^{R 15}$ \\
\hline 391257104173601 & DENV & DENV 16 & $6,216.99$ & 2015-04-09 & $6,216.58$ & 2015-06-08 & $6,217.02$ & 2016-10-06 & $6,216.61$ & 2016-04-04 & $6,216.92$ & 2017-03-31 & $6,216.54$ & $2017-12-12$ & $6,216.86$ & 2018-04-12 & $6,216.68$ & 2018-06-06 & -0.13 \\
\hline 391811104140301 & DENV & DENV 15 & $5,865.73$ & 2015-06-05 & $5,862.98$ & 2015-04-03 & $5,865.70$ & 2016-06-01 & $5,863.98$ & 2016-10-03 & $5,864.95$ & 2017-01-30 & $5,863.51$ & 2017-03-30 & $5,865.77$ & 2018-02-16 & $5,863.35$ & 2018-06-04 & $2.12^{\mathrm{R} 15}$ \\
\hline 391821104270601 & DENV & DENV 14 & $6,404.46$ & 2015-06-11 & $6,401.05$ & 2015-10-05 & $6,404.98$ & 2016-06-03 & $6,399.79$ & 2016-12-08 & $6,403.82$ & 2017-04-13 & $6,399.61$ & 2017-10-04 & $6,402.64$ & 2018-02-14 & $6,399.54$ & 2018-06-07 & -1.66 \\
\hline 391851104204501 & DENV & DENMAS05 & $5,833.79$ & $-06-05$ & 5,7 & 2015-04-03 & 66 & 26 & 5 , & 20 & $5,832.36$ & 2017-02-06 & $5,829.88$ & $2017-08-07$ & $5,832.53$ & 2018-04-18 & $5,810.70$ & 2018-02-16 & $52.83^{\mathrm{pl}}$ \\
\hline 301 & ENV & NI & $5,915.61$ & 2015-06-10 & $5,885.59$ & $\begin{array}{l}2015-08-04 \\
2015-10-08\end{array}$ & 11.90 & $16-02-19$ & 7.60 & $6-06-06$ & -- & -- & -- & -- & -- & -- & -- & -- & M \\
\hline 393350104151701 & DENV & DENV 12 & $5,473.64$ & 2015-12-04 & $5,473.34$ & 2015-04-03 & $5,474.02$ & 2016-10-03 & $5,473.34$ & 2016-04-01 & $5,473.72$ & 2017-03-30 & $5,473.13$ & $2017-12-06$ & $5,473.52$ & 2018-06-04 & $5,473.15$ & 2018-04-18 & -0.19 \\
\hline
\end{tabular}


Table 3. Summary of discrete groundwater-level elevations, April 2015 to June 2018, Elbert County, Colorado.-Continued

[See table 2 and figure 2 for well locations. R15, indicates April 2015 water level has status R (recently pumped). R18, indicates April 18 water level has status R (recently pumped). P15, indicates April 2015 water level has status P (pumping). P18, indicates April 2018 water level has status P (pumping). See "Study Methods" section for details. ft, foot; NAVD 88, North American Vertical Datum of 1988; UDAW, upper Dawson aquifer; LDAW, lower Dawson aquifer; DENV, Denver aquifer; ARAP, Arapahoe aquifer; LARA, Laramie-Fox Hills aquifer; --, no measurements; M, site missing either April 2015 or April 2018 measurement]

\begin{tabular}{|c|c|c|c|c|c|c|c|c|c|c|c|c|c|c|c|c|c|c|c|}
\hline \multirow[b]{2}{*}{$\begin{array}{c}\text { Site } \\
\text { identification } \\
\text { number }\end{array}$} & \multirow[b]{2}{*}{ Aquifer } & \multirow[b]{2}{*}{$\begin{array}{c}\text { Common } \\
\text { name }\end{array}$} & \multicolumn{4}{|c|}{2015} & \multicolumn{4}{|c|}{2016} & \multicolumn{4}{|c|}{2017} & \multicolumn{4}{|c|}{ January 2018 to June 2018} & \multirow[b]{2}{*}{$\begin{array}{c}\text { Relative } \\
\text { Change } \\
\text { April } 2015 \\
\text { to April } \\
2018 \text { (ft) }\end{array}$} \\
\hline & & & $\begin{array}{l}\text { Maximum } \\
\text { ground- } \\
\text { water } \\
\text { elevation } \\
\text { (ft above } \\
\text { NAVD 88) }\end{array}$ & $\begin{array}{c}\text { Date of } \\
\text { maximum } \\
\text { measurement }\end{array}$ & $\begin{array}{l}\text { Minimum } \\
\text { ground- } \\
\text { water } \\
\text { elevation } \\
\text { (ft above } \\
\text { NAVD 88) }\end{array}$ & $\begin{array}{c}\text { Date of } \\
\text { minimum } \\
\text { measurement }\end{array}$ & $\begin{array}{l}\text { Maximum } \\
\text { ground- } \\
\text { water } \\
\text { elevation } \\
\text { (ft above } \\
\text { NAVD 88) }\end{array}$ & $\begin{array}{c}\text { Date of } \\
\text { maximum } \\
\text { measure- } \\
\text { ment }\end{array}$ & $\begin{array}{l}\text { Minimum } \\
\text { ground- } \\
\text { water } \\
\text { elevation } \\
\text { (ft above } \\
\text { NAVD 88) }\end{array}$ & $\begin{array}{c}\text { Date of } \\
\text { minimum } \\
\text { measure- } \\
\text { ment }\end{array}$ & $\begin{array}{l}\text { Maximum } \\
\text { ground- } \\
\text { water } \\
\text { elevation } \\
\text { (ft above } \\
\text { NAVD 88) }\end{array}$ & $\begin{array}{c}\text { Date of } \\
\text { maximum } \\
\text { measure- } \\
\text { ment }\end{array}$ & $\begin{array}{l}\text { Minimum } \\
\text { ground- } \\
\text { water } \\
\text { elevation } \\
\text { (ft above } \\
\text { NAVD 88) }\end{array}$ & $\begin{array}{c}\text { Date of } \\
\text { minimum } \\
\text { measure- } \\
\text { ment }\end{array}$ & $\begin{array}{l}\text { Maximum } \\
\text { ground- } \\
\text { water } \\
\text { elevation } \\
\text { (ft above } \\
\text { NAVD 88) }\end{array}$ & $\begin{array}{l}\text { Date of } \\
\text { maximum } \\
\text { measure- } \\
\text { ment }\end{array}$ & $\begin{array}{l}\text { Minimum } \\
\text { ground- } \\
\text { water } \\
\text { elevation } \\
\text { (ft above } \\
\text { NAVD 88) }\end{array}$ & $\begin{array}{c}\text { Date of } \\
\text { minimum } \\
\text { measure- } \\
\text { ment }\end{array}$ & \\
\hline 390800104172601 & ARAP & ARAP 8 & $6,050.72$ & 2015-08-03 & $6,041.77$ & 2015-10-01 & $6,051.45$ & 2016-08-11 & $6,048.77$ & 2016-02-12 & $6,048.92$ & 2017-08-07 & $6,044.14$ & 2017-06-15 & $6,048.37$ & 2018-02-13 & $6,023.39$ & 2018-06-06 & $-4.23^{\mathrm{p} 18}$ \\
\hline 391208104053301 & ARAP & ARAP 7 & $5,985.15$ & 2015-12-10 & $5,984.64$ & 2015-08-03 & $5,984.83$ & 2016-02-12 & $5,984.24$ & 2016-06-02 & $5,985.17$ & 2017-03-31 & $5,984.58$ & 2017-08-07 & $5,984.62$ & 2018-02-13 & $5,977.49$ & 2018-04-12 & -7.57 \\
\hline 391740104072401 & ARAP & ARAPMAS27 & $5,810.69$ & 2015-08-03 & $5,810.28$ & 2015-04-09 & $5,811.22$ & 2016-06-02 & $5,810.15$ & 2016-10-06 & $5,811.58$ & $2017-08-03$ & $5,810.62$ & 2017-06-15 & $5,811.62$ & 2018-04-12 & $5,811.53$ & 2018-02-13 & 1.34 \\
\hline 391834104205601 & ARAP & ARAPMAS22 & $5,830.48$ & 2015-06-05 & $5,828.36$ & 2015-12-03 & $5,830.09$ & 2016-04-01 & $5,826.99$ & 2016-08-09 & $5,829.35$ & 2017-02-06 & $5,827.80$ & 2017-10-05 & $5,829.25$ & 2018-02-16 & $5,825.86$ & 2018-06-04 & -1.54 \\
\hline 391946104114501 & ARAP & ARAP 6 & $5,867.06$ & 2015-06-05 & $5,866.84$ & 2015-08-06 & $5,867.22$ & 2016-06-01 & $5,860.62$ & 2016-10-03 & $5,867.74$ & 2017-12-06 & $5,866.82$ & 2017-01-30 & $5,868.06$ & 2018-04-18 & $5,867.85$ & 2018-06-04 & 1.15 \\
\hline 392400104150601 & ARAP & ARAPMAS 28 & $5,715.55$ & 2015-06-05 & $5,714.33$ & 2015-10-02 & $5,716.07$ & 2016-06-01 & $5,714.53$ & 2016-08-08 & $5,715.85$ & 2017-03-30 & $5,714.46$ & 2017-07-31 & $5,715.37$ & 2018-02-16 & $5,715.07$ & 2018-06-04 & 0.34 \\
\hline 392434104142701 & ARAP & ARAP 5 & $5,749.14$ & 2015-06-05 & $5,748.19$ & 2015-10-02 & $5,748.81$ & 2016-02-11 & $5,745.81$ & 2016-08-08 & $5,747.79$ & $2017-01-30$ & $5,746.86$ & 2017-10-05 & $5,747.09$ & $2018-02-16$ & $5,746.81$ & 2018-06-04 & -1.45 \\
\hline 393225104073601 & ARAP & AP 4 & 5 & 06 & 21 & $2015-10-02$ & 5 , & 2016-06-01 & 5 , & 3 & 95 & 30 & 5 & $\begin{array}{l}2017-06-22 \\
2017-10-05\end{array}$ & 35 & $2018-0$ & 421.41 & 2018-06-04 & $-0.12^{\mathrm{R} 15}$ \\
\hline 393251104073701 & ARAP & ARAP 3 & $5,379.79$ & 2015-12-04 & $5,378.16$ & 2015-10-02 & $5,380.43$ & 2016-02-11 & $5,368.19$ & 2016-10-03 & $5,377.17$ & 2017-12-06 & $5,373.51$ & 2017-07-31 & $5,377.99$ & 2018-02-16 & $5,377.37$ & 2018-06-04 & -1.43 \\
\hline 390817104040301 & LARA & LARA 7 & $5,801.43$ & 2015-06-08 & $5,796.11$ & 2015-10-01 & $5,800.89$ & 2016-06-02 & $5,798.19$ & 2016-10-06 & $5,800.57$ & 2017-02-02 & $5,797.01$ & 2017-06-15 & $5,799.89$ & 2018-02-13 & $5,798.11$ & 2018-06-06 & $-1.17^{\mathrm{R} 18}$ \\
\hline 391609104014001 & LARA & LARA 6 & $5,609.56$ & 2015-06-08 & $5,608.72$ & 2015-08-03 & $5,609.37$ & 2016-06-02 & $5,607.55$ & 2016-10-06 & $5,608.54$ & 2017-03-31 & $5,607.01$ & 2017-10-12 & $5,607.85$ & 2018-04-12 & $5,606.95$ & 2018-06-06 & -1.18 \\
\hline 391621104012001 & LARA & LARA 5 & $5,606.37$ & 2015-06-08 & $5,605.37$ & 2015-10-01 & $5,606.05$ & 2016-02-12 & $5,604.35$ & 2016-10-06 & $5,605.37$ & 2017-03-31 & $5,603.90$ & 2017-10-12 & $5,604.74$ & 2018-04-12 & $5,603.86$ & 2018-06-06 & -1.09 \\
\hline 392616103591001 & LARA & LARA 3 & $5,413.41$ & 2015-12-04 & $5,402.01$ & 2015-06-05 & $5,414.08$ & 2016-04-01 & $5,405.35$ & 2016-08-08 & $5,414.19$ & 2017-03-30 & $5,403.92$ & 2017-07-31 & $5,413.61$ & 2018-04-18 & $5,412.88$ & 2018-02-16 & 2.92 \\
\hline 392635103590001 & LARA & LARA 4 & $5,404.00$ & 2015-12-04 & $5,387.85$ & 2015-06-05 & $5,404.77$ & 2016-06-01 & $5,393.36$ & 2016-08-08 & $5,404.57$ & 2017-03-30 & $5,390.35$ & $2017-06-23$ & -- & -- & -- & -- & M \\
\hline
\end{tabular}


Table 4. Statistically significant trends in discrete static groundwater-level elevations, April 2015 to June 2018, Elbert County, Colorado.

[See table 2 and figure 2 for well locations. ft, foot; $p$-value, probability value; $\mathrm{R}^{2}$, coefficient of determination; UDAW, upper Dawson aquifer; LDAW, lower Dawson aquifer; DENV, Denver aquifer; ARAP, Arapahoe aquifer; LARA, Laramie-Fox Hills aquifer; <, less than]

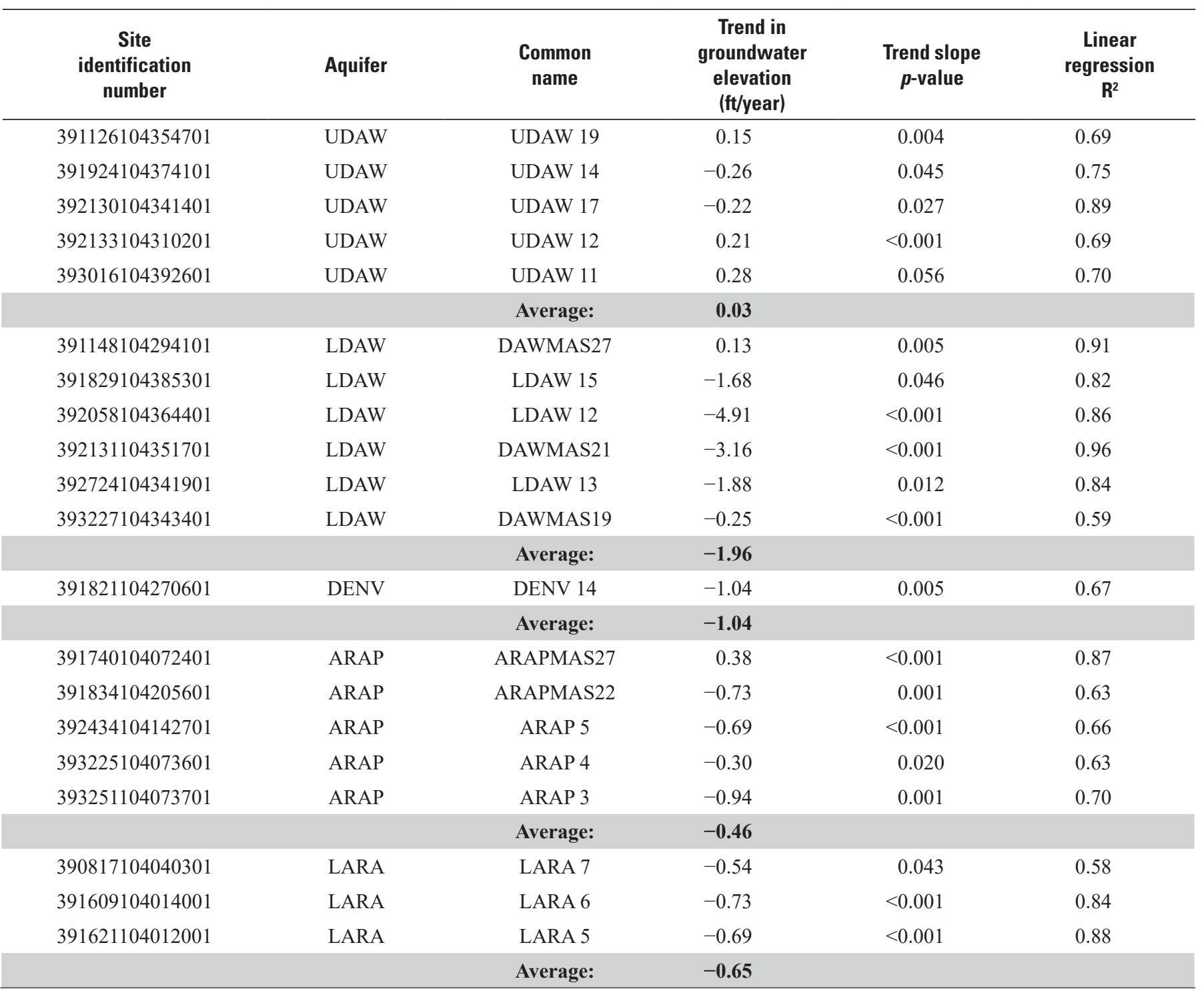

a strong seasonal pattern (table 5). Trends in the continuous daily maximum groundwater-level elevations agreed with the trend direction and magnitude calculated from discrete groundwater-level elevations, except for LARA 3, which did not have a significant trend in discrete groundwater-level elevations, and DENV 12, which did not have significant trends for discrete or continuous groundwater-level elevations (table 6).

The trend for discrete data at LARA 3 (appendix fig. 2.33) was not significant, but indicates a slightly rising groundwater level, whereas the trend for continuous data indicates a slightly declining level. The disagreement between continuous and discrete data trends may be caused by the pronounced and nonuniform seasonal pattern. Adjacent to the well, agricultural irrigation operations strongly affect groundwater levels in summer. Discrete measurements at a nearby well (LARA 4, appendix fig. 2.34) show a similar drop in summer groundwater levels. The hydrograph for LARA 3 (fig. 2.33) shows daily maximum groundwater-level elevation data, highlighting the additional information that continuous measurements provide about groundwater-level changes between discrete measurements.

The two wells in the upper Dawson aquifer with continuous measurements had differing trend directions. UDAW 14 (appendix fig. 2.22) exhibited a negative trend of $-0.31 \mathrm{ft} /$ year, whereas UDAW 12 (appendix fig. 2.28) exhibited a slightly positive trend of $0.06 \mathrm{ft} /$ year (table 6 ). This is consistent with 


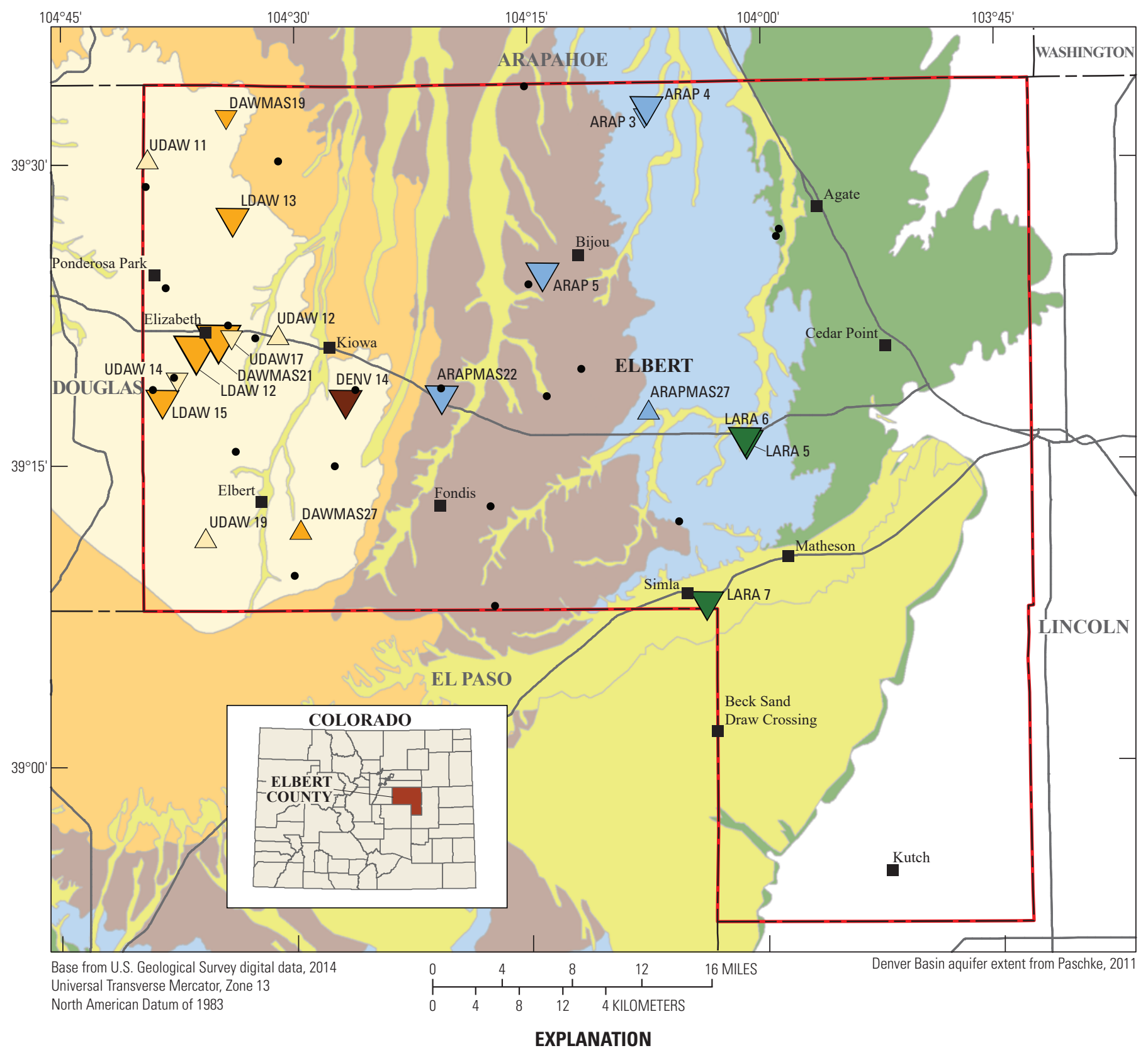

\begin{tabular}{|c|c|c|}
\hline Aquifer extent & Trend & Well indicating aquifer \\
\hline Alluvial deposits & $\triangle$ Positive & $\triangle \quad$ Upper Dawson \\
\hline Upper Dawson & $\nabla$ Negative & Lower Dawson \\
\hline Lower Dawson & Slope, in feet & $\triangle$ Arapahoe \\
\hline Denver & per year & Laramie Fox Hills \\
\hline Arapahoe & $\triangle 0.13$ to 0.5 & \\
\hline Laramie-Fox Hills & 0.5 to 2.0 & \\
\hline
\end{tabular}

Figure 5. Distribution of significant trends in discrete groundwater-level elevations, by aquifer, Elbert County, Colorado. 


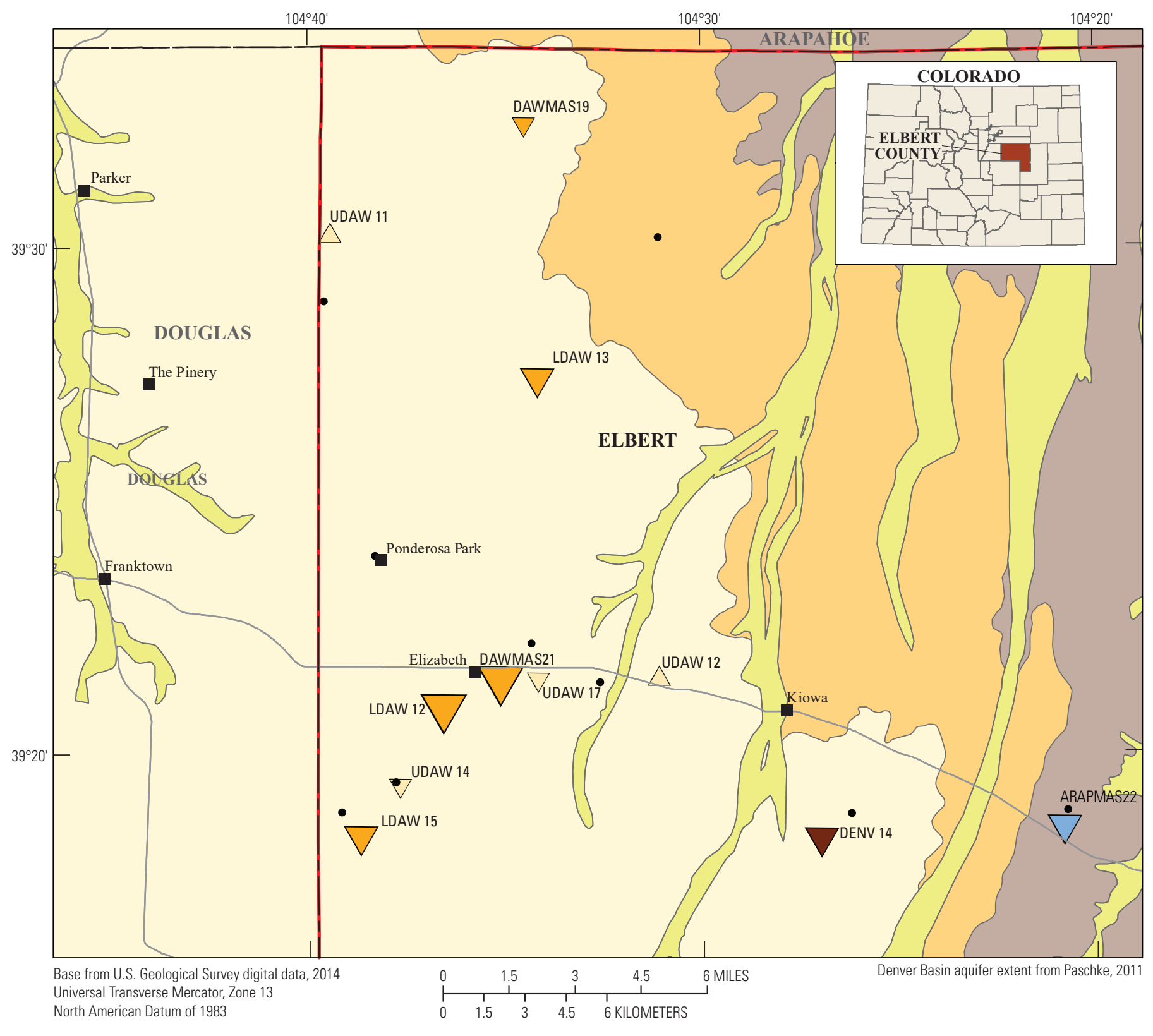

EXPLANATION

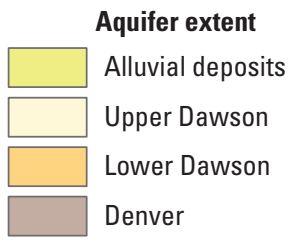

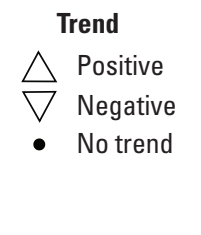

Slope, in feet

Well indicating aquifer of completion

Elbert County study area boundary

Figure 6. Distribution of significant trends in discrete groundwater-level elevations in the upper Dawson and lower Dawson aquifers near the towns of Elizabeth and Kiowa, Elbert County, Colorado. 
Table 5. Statistical summary of continuous daily maximum groundwater-level elevations, January 2016 to June 2018, Elbert County, Colorado.

[See table 2 and figure 2 for well locations, and appendix 2 for complete hydrographs. ft, foot; NAVD 88, North American Vertical Datum of 1988; UDAW, upper Dawson aquifer; LDAW, lower Dawson aquifer; DENV, Denver aquifer; LARA, Laramie-Fox Hills aquifer; Jan.; January; Feb., February; Mar., March; Apr., April; Aug., August; Sep,. September; Oct., October; Nov., November; Dec., December]

\begin{tabular}{|c|c|c|c|c|c|c|c|c|c|c|c|c|c|c|}
\hline \multirow[b]{2}{*}{$\begin{array}{c}\text { Site } \\
\text { identification } \\
\text { number }\end{array}$} & \multirow[b]{2}{*}{ Aquifer } & \multirow[b]{2}{*}{$\begin{array}{c}\text { Common } \\
\text { name }\end{array}$} & \multicolumn{4}{|c|}{2016} & \multicolumn{4}{|c|}{2017} & \multicolumn{4}{|c|}{ January 2018 to June 2018} \\
\hline & & & $\begin{array}{l}\text { Maximum } \\
\text { ground- } \\
\text { water } \\
\text { elevation } \\
\text { (ft above } \\
\text { NAVD 88) }\end{array}$ & $\begin{array}{l}\text { Month of } \\
\text { maximum }\end{array}$ & $\begin{array}{l}\text { Minimum } \\
\text { ground- } \\
\text { water } \\
\text { elevation } \\
\text { (ft above } \\
\text { NAVD 88) }\end{array}$ & $\begin{array}{l}\text { Month of } \\
\text { minimum }\end{array}$ & $\begin{array}{l}\text { Maximum } \\
\text { ground- } \\
\text { water } \\
\text { elevation } \\
\text { (ft above } \\
\text { NAVD 88) }\end{array}$ & $\begin{array}{l}\text { Month of } \\
\text { maximum }\end{array}$ & $\begin{array}{l}\text { Minimum } \\
\text { ground- } \\
\text { water } \\
\text { elevation } \\
\text { (ft above } \\
\text { NAVD 88) }\end{array}$ & $\begin{array}{l}\text { Month of } \\
\text { minimum }\end{array}$ & $\begin{array}{l}\text { Maximum } \\
\text { ground- } \\
\text { water } \\
\text { elevation } \\
\text { (ft above } \\
\text { NAVD 88) }\end{array}$ & $\begin{array}{l}\text { Month of } \\
\text { maximum }\end{array}$ & $\begin{array}{l}\text { Minimum } \\
\text { ground- } \\
\text { water } \\
\text { elevation } \\
\text { (ft above } \\
\text { NAVD 88) }\end{array}$ & $\begin{array}{l}\text { Month of } \\
\text { minimum }\end{array}$ \\
\hline 391924104374101 & UDAW & UDAW 14 & $6,601.24$ & May & $6,597.45$ & Aug. & $6,601.22$ & Feb. & $6,596.95$ & Sep. & $6,600.81$ & Feb. & $6,598.22$ & June \\
\hline 392133104310201 & UDAW & UDAW 12 & $6,441.32$ & Dec. & $6,440.00$ & Nov. & $6,441.45$ & Apr. & $6,440.13$ & Oct. & $6,441.53$ & Apr. & $6,440.69$ & Jan. \\
\hline 392058104364401 & LDAW & LDAW 12 & $6,443.69$ & May & $6,413.89$ & Sep. & $6,438.73$ & Mar. & $6,411.04$ & Oct. & $6,436.40$ & Apr. & $6,419.50$ & June \\
\hline 393227104343401 & LDAW & DAWMAS19 & $6,046.71$ & Apr. & $6,045.35$ & Nov. & $6,046.69$ & Mar. & $6,044.75$ & July & $6,046.49$ & Feb. & $6,045.29$ & June \\
\hline 393350104151701 & DENV & DENV 12 & $5,474.27$ & Nov. & $5,473.27$ & Nov. & $5,474.14$ & Mar. & $5,473.24$ & Oct. & $5,474.30$ & Feb. & $5,473.29$ & Apr. \\
\hline 392616103591001 & LARA & LARA 3 & $5,414.46$ & May & $5,402.33$ & Aug. & $5,414.47$ & Apr. & $5,401.91$ & July & $5,413.85$ & Apr. & $5,412.81$ & Feb. \\
\hline
\end{tabular}

Table 6. Trends in continuous and discrete daily maximum groundwater-level elevations, 2015 to June 2018, Elbert County Colorado.

[See table 2 and figure 2 for well locations. Negative trend values indicate negative trend; positive trend values indicate positive trend. Discrete groundwater level trends from table 4 and table 3.1. POR, period of record; ft, foot; $p$-value, probability value; $\mathrm{R}^{2}$, coefficient of determination; UDAW, upper Dawson aquifer; LDAW, lower Dawson aquifer; DENV, Denver aquifer; LARA, Laramie-Fox Hills aquifer; italics, indicates trend is not signifcant; <, less than]

\begin{tabular}{|c|c|c|c|c|c|c|c|c|}
\hline $\begin{array}{c}\text { Site } \\
\text { identification } \\
\text { number }\end{array}$ & Aquifer & $\begin{array}{c}\text { Common } \\
\text { name }\end{array}$ & $\begin{array}{l}\text { POR } \\
\text { start } \\
\text { date }\end{array}$ & $\begin{array}{l}\text { POR } \\
\text { end } \\
\text { date }\end{array}$ & $\begin{array}{c}\text { Trend } \\
\text { test }\end{array}$ & $\begin{array}{c}\text { Trend in } \\
\text { groundwater } \\
\text { elevation } \\
\text { (ft/year) }\end{array}$ & $\begin{array}{c}\text { Trend } \\
\text { slope } \\
p \text {-value }\end{array}$ & $\begin{array}{c}\text { Linear } \\
\text { regression } \\
\mathbf{R}^{2}\end{array}$ \\
\hline \multirow{2}{*}{391924104374101} & \multirow{2}{*}{ UDAW } & \multirow{2}{*}{ UDAW 14} & \multirow{2}{*}{ 2015-10-06 } & \multirow{2}{*}{ 2018-06-07 } & Continuous groundwater elevation trend: & -0.31 & $<0.001$ & 0.85 \\
\hline & & & & & Discrete groundwater elevation trend: & -0.26 & 0.045 & 0.75 \\
\hline \multirow{2}{*}{392133104310201} & \multirow{2}{*}{ UDAW } & \multirow{2}{*}{ UDAW 12} & \multirow{2}{*}{$2015-08-13$} & \multirow{2}{*}{ 2018-06-05 } & Continuous groundwater elevation trend: & 0.06 & $<0.001$ & 0.48 \\
\hline & & & & & Discrete groundwater elevation trend: & 0.21 & $<0.001$ & 0.69 \\
\hline \multirow{2}{*}{392058104364401} & \multirow{2}{*}{ LDAW } & \multirow{2}{*}{ LDAW 12} & \multirow{2}{*}{ 2015-08-08 } & \multirow{2}{*}{ 2018-06-07 } & Continuous groundwater elevation trend: & -3.73 & $<0.001$ & 0.90 \\
\hline & & & & & Discrete groundwater elevation trend: & -4.91 & $<0.001$ & 0.86 \\
\hline \multirow{2}{*}{393227104343401} & \multirow{2}{*}{ LDAW } & \multirow{2}{*}{ DAWMAS19 } & \multirow{2}{*}{ 2015-11-07 } & \multirow{2}{*}{ 2018-06-07 } & Continuous groundwater elevation trend: & -0.18 & $<0.001$ & 0.49 \\
\hline & & & & & Discrete groundwater elevation trend: & -0.25 & $<0.001$ & 0.59 \\
\hline \multirow{2}{*}{393350104151701} & \multirow{2}{*}{ DENV } & \multirow{2}{*}{ DENV 12} & \multirow{2}{*}{ 2016-02-12 } & \multirow{2}{*}{ 2018-06-03 } & Continuous groundwater elevation trend: & -0.03 & $<0.001$ & 0.11 \\
\hline & & & & & Discrete groundwater elevation trend: & -0.04 & 0.354 & 0.01 \\
\hline \multirow{2}{*}{392616103591001} & \multirow{2}{*}{ LARA } & \multirow{2}{*}{ LARA 3} & \multirow{2}{*}{ 2015-08-07 } & \multirow{2}{*}{$2018-06-03$} & Continuous groundwater elevation trend: & -0.61 & $<0.001$ & 0.43 \\
\hline & & & & & Discrete groundwater elevation trend: & 0.33 & 0.626 & 0.44 \\
\hline
\end{tabular}


trends associated with wells in the upper Dawson aquifer that had only discrete measurements; these wells had positive and negative trends (table 4). The two wells in the lower Dawson aquifer (LDAW12 and DAWMAS19; table 6) have negative trends in continuous groundwater-level elevations that are consistent with trends for discrete groundwater-level elevations but vary in magnitude. LDAW 12 (appendix fig. 2.24), close to the town of Elizabeth, Colo., had a negative trend of $-3.73 \mathrm{ft} / \mathrm{year}$, whereas farther north DAWMAS19 (appendix fig. 2.40) had a negative trend of only $-0.18 \mathrm{ft}$ /year (table 6, fig. 6).

\section{Potentiometric-Surface and Difference Maps}

Potentiometric-surface maps were derived from static groundwater-level measurements made during April 2015 and April 2018 in the upper and lower Dawson aquifers. Only wells with a static April 2015 and a static April 2018 measurement were used to derive the maps; to remove the effect of pumping on the derivation and to keep the derivation points used by ArcMap Spatial Analyst Toolbox consistent between measurement periods. The potentiometric surface as 100 -ft-interval contours for April 2018, and the differences in hydraulic head between April 2015 and April 2018, in the upper and lower Dawson aquifers are shown in figures 7 and 8 , respectively.

Groundwater flow in the upper and lower Dawson aquifers in western Elbert County, based on the derived potentiometric-surface maps, is generally from south to north. The difference maps of hydraulic head show that the two aquifers had lower hydraulic head values along the western border of Elbert County in April 2018 than April 2015 (figs. 7 and fig. 8). Elsewhere in the upper Dawson aquifer, the hydraulic head values were generally the same or slightly elevated, especially northeast of Elizabeth, Colo., and extending towards the northeastern extent of the aquifer (fig. 7). The lower Dawson aquifer had lower hydraulic head values in most of the study area, with the greatest differences near Elizabeth, Colo., and extending towards the western border (fig. 8). Only small parts of the lower Dawson aquifer between Elizabeth and Kiowa, Colo., and southeast of Elbert, Colo., had higher head values in April 2018 than in April 2015 (fig. 8).

Negative trends in groundwater-level elevations in the upper and lower Dawson aquifers in the western part of the county could coincide with increased development in Elbert County and Douglas County. According to the CDWR well permit database (Colorado Department of Natural Resources, 2018), from January 2015 to June 2018, 335 new domestic water-supply wells were constructed in the upper and lower Dawson aquifers, mostly in eastern Douglas County and western Elbert County. Parts of the upper and lower Dawson aquifers in Elbert County where levels are rising, coinciding with positive changes and trends, could indicate the presence of outcrops of the aquifers that are localized recharge zones, especially towards the outer extent of the lower Dawson aquifer where unconfined flow conditions may exist and in the upper Dawson aquifer, which is unconfined throughout and has the greater recharge (Paschke, 2011).

\section{Future Work}

Results from this study can be used to focus future monitoring efforts and aid local water-resource managers with decisions on water use within Elbert County. Continued monitoring and analysis are needed to confirm whether the groundwaterlevel elevation comparisons and trends from this study persist beyond the study period. Continued monitoring and more static measurements at wells with no significant trend in this study may lead to significant trends in the future by providing more data points as input to linear regressions. Additionally, instrumenting more wells with continuous groundwater-level monitors would provide a more in-depth record of patterns in groundwater levels between discrete measurements. Future monitoring efforts can focus less on aquifers with small groundwater-level declines and areas with little development in the eastern part of the county and focus more on aquifers with substantial negative trends and areas experiencing greater development. Additional monitoring could include soliciting more homeowners for well access, measuring water levels in or instrumenting existing CDWR wells, or installing new monitoring wells in the county. A regional study and analysis that includes groundwater-level data from other monitoring networks in the Denver Basin aquifer system could provide a better understanding of how groundwater levels are changing beyond the Elbert County border and how those changes could affect groundwater resources in Elbert County. The regional study could produce regional water-level trend maps and regional potentiometric surface and hydraulic-head difference maps. It could provide additional calibration data, which could be used to update and improve the Denver Basin groundwater model.

\section{Summary}

Residents in Elbert County, Colorado, rely on groundwater withdrawals to meet public and domestic water-supply needs throughout the county. Withdrawals from the five bedrock aquifers in the Denver Basin aquifer system (lower Dawson, upper Dawson, Denver, Arapahoe, and LaramieFox Hills) in Elbert County and in neighboring counties have increased to meet the demand of a growing population. The U.S. Geological Survey, in cooperation with the Elbert County Board of County Commissioners, began a study in 2015 to establish a network of wells and measure groundwater levels on a bi-monthly interval. Six of the 42 wells in the study were instrumented with pressure transducers to record groundwater levels hourly. The purposes of this study were to measure groundwater levels between April 2015 and June 2018 and analyze groundwater-level elevation changes and trends throughout the county.

Measurements made in all wells were used to calculate changes and trends in groundwater-level elevations in all five Denver Basin bedrock aquifers within Elbert County. Relative changes between April 2015 and April 2018 were calculated using discrete measurements. Trends were calculated using 


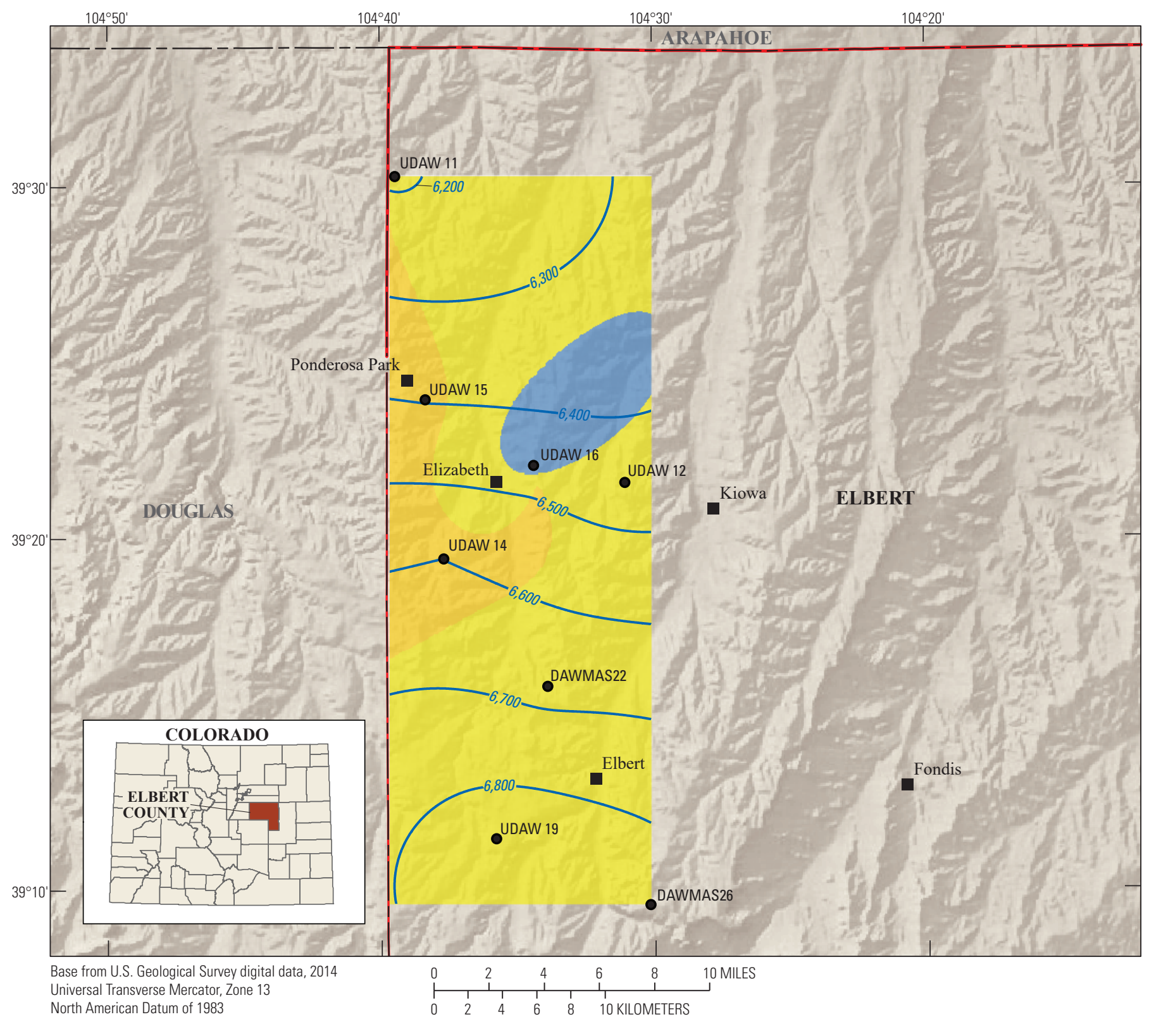

\section{EXPLANATION}

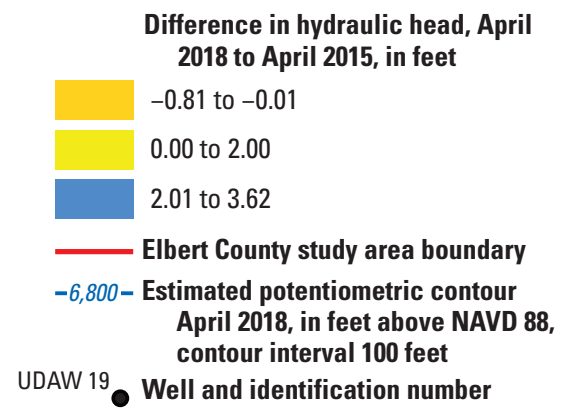

Figure 7. Estimated potentiometric surface of the upper Dawson aquifer in April 2018 and change in hydraulic head between April 2015 and April 2018, western Elbert County, Colorado. 


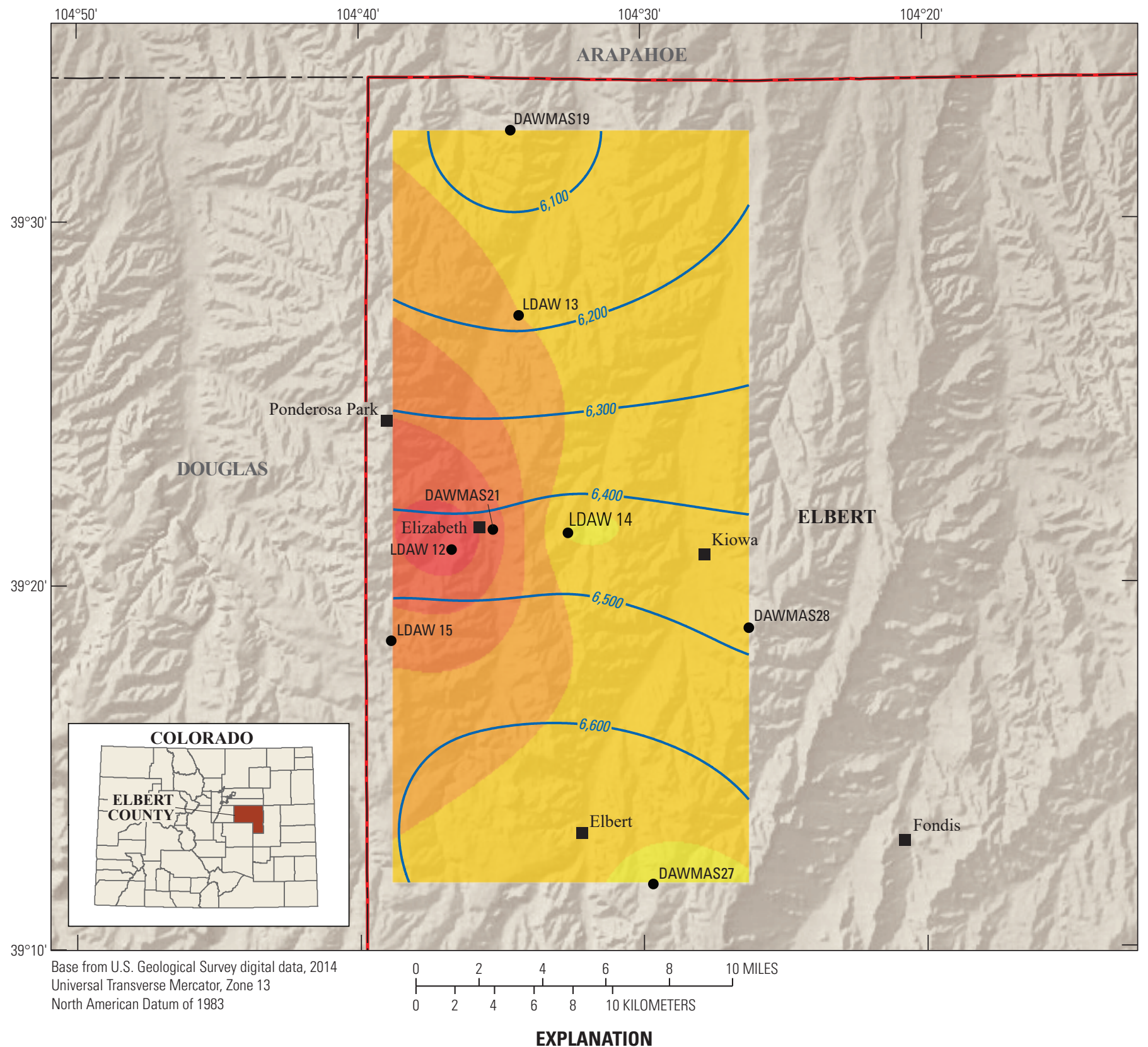

Difference in hydraulic head, April 2018 to April 2015, in feet

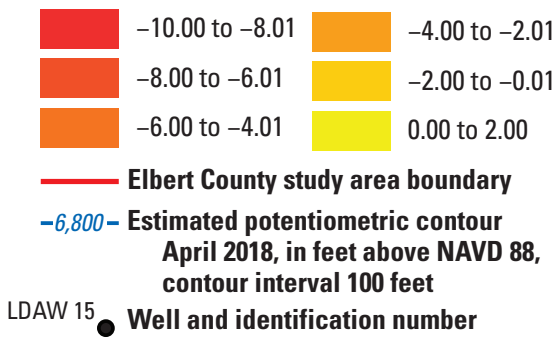

Figure 8. Estimated potentiometric surface of the lower Dawson aquifer in April 2018 and change in hydraulic head between April 2015 and April 2018, western Elbert County, Colorado. 
linear regression models, static discrete groundwater-level elevations, and continuous daily maximum groundwater-level elevations. The spatial distribution of hydraulic head in April 2018, and the relative changes in hydraulic head from April 2015 to April 2018, were mapped in the upper Dawson and lower Dawson aquifers.

All five aquifers had wells with a rise in groundwaterlevel elevation and wells with a decline in groundwaterlevel elevation, based on the relative change in the groundwater-level elevation between the April 2015 and April 2018 measurements. The upper Dawson, lower Dawson, Denver, Arapahoe, and Laramie-Fox Hills aquifers had average trends of 0.03 foot per year (ft/year), $-1.96 \mathrm{ft} /$ year, $-1.04 \mathrm{ft} /$ year, $-0.46 \mathrm{ft} /$ year, and $-0.65 \mathrm{ft} /$ year, respectively. Trends in groundwater-level elevations in the upper Dawson aquifer were not consistent. Some wells had declining levels and some rising levels, but all trends in this aquifer were within a range of $\pm 1 \mathrm{ft} /$ year. The heterogeneity in trend direction and location could be an indication of local zones of recharge. The magnitude of negative groundwater-level elevation trends in the lower Dawson aquifer had a larger range than the other four aquifers, ranging from $-0.25 \mathrm{ft} /$ year to $-4.91 \mathrm{ft} /$ year, with 4 of 5 negative trends in excess of $1 \mathrm{ft} /$ year and an average discrete trend of $-1.96 \mathrm{ft} /$ year. Negative trends in the lower Dawson aquifer were concentrated along the western border of Elbert County, which is central to the aquifer's extent. The only positive trend in groundwater-level elevation in the lower Dawson aquifer occurred for a well at the eastern edge of the aquifer's extent. Of the seven wells where water levels were monitored in the Denver aquifer, a statistically significant trend was observed in only one well (-1.04 ft/year). A longer study period and more static measurements could increase the frequency of significant trends. Like trends for the upper Dawson aquifer, groundwater-level elevation trends at wells in the Arapahoe aquifer and Laramie-Fox Hills aquifer were all within a range of $\pm 1 \mathrm{ft} /$ year. Trends in continuous groundwaterlevel elevations were in agreement with significant trends in discrete groundwater-level elevations. Potentiometric-surface maps of the upper and lower Dawson aquifers for April 2015 and April 2018 show that differences in hydraulic head from the two measurement periods were greatest along the western part of Elbert County.

Results of this study can be used by local water-resource managers to make decisions about water use within Elbert County and could be used to guide future groundwater monitoring options. Results also could be used for a regional study of groundwater-level elevations in the Denver Basin aquifer system to provide additional calibration data for the Denver Basin groundwater flow model.

\section{References Cited}

Barkmann, P.E., 2004, Vertical hydraulic conductivity measurements in the Denver Basin, Colorado: The Mountain Geologist, v. 41, no. 4, p. 169-183.

Colorado Department of Natural Resources, 2018, CWCB/DWR Colorado's Decision Support Systems: Well Permits: Denver, Colo., Colorado Department of Natural Resources, accessed October 2, 2018, at https://dnrweb.state.co.us/cdss/WellPermits.

Crifasi, R.R., 1992, Alluvial architecture of Laramide orogenic sediments Denver Basin, Colorado: The Mountain Geologist, v. 29, p. 19-27.

Cunningham, W.L., and Schalk, C.W., comps., 2011, Groundwater technical procedures of the U.S. Geological Survey: U.S. Geological Survey Techniques and Methods 1-A1, $151 \mathrm{p}$.

Douglas County, 2018, Douglas County growth and development profile, 2 p.: Castle Rock, Colo., Douglas County, accessed July 27, 2018, at https://www.douglas.co.us/documents/douglascounty-growth-and-development-profile.pdf.

Elbert County, 2009, Subdivision Regulations, Community and Development Services, Kiowa, Colo., 84 p., accessed July 27, 2018, at http://www.elbertcounty-co.gov/docs/ Subdivision_Regs.pdf.

Elbert County Planning Commission, 2018, Elbert County Comprehensive Plan Update, Kiowa, Colo., 77 p., accessed July 13, 2018, at http://www.elbertcounty-co.gov/5\%20 -\%20CDS/Elbert\%20County_Comp\%20Plan_2018_ FINAL.pdf.

Esri Inc., 1999-2016, ArcGIS Desktop 10.5, Version 10.5.0.6491: Redlands, Calif., Esri Inc.

Everett, R.R., 2014, Groundwater levels in the Denver Basin bedrock aquifers of Douglas County, Colorado, 2011-2013: U.S. Geological Survey Scientific Investigations Report 2014-5172, 45 p., http://dx.doi.org/10.3133/sir20145172.

Fenneman, N.M., 1931, Physiography of the western United States: New York, McGraw Hill, 534 p.

Flor, A.D., 2017, Groundwater levels in the Denver Basin bedrock aquifers 2017: Kiowa, Colo., Colorado Department of Natural Resources Division of Water Resources, 210 p.

Forsgren Associates Inc., 2018, Elbert County, CO, rural water supply study, (revised draft version): Englewood, Colo., Forsgren Associates Inc., 58 p., accessed July 12, 2018, at http://www.elbertcounty-co.gov/5\%20-\%20CDS/Elbert\%20 County\%20Rural\%20Water\%20Supply\%20Study-February $\% 202018 \% 20$ draft $\% 20$ report_ii.pdf. 
Freeman, L.A., Carpenter, M.C., Rosenberry, D.O., Rousseau, J.P., Unger, R. and McLean, J.C. 2004, Use of submersible pressure transducers in water-resources investigations: U.S. Geological Survey Techniques of Water-Resources Investigations, book 8, chap. A3, 52 p., accessed January 1, 2013 at https://doi.org/10.3133/twri08A3.

Helsel, D.R., and Hirsch, R.M., 2002, Statistical methods in water resources: U.S. Geological Survey Techniques of WaterResources Investigations, book 4, chap. A3, 522 p. [Also available at https://pubs.er.usgs.gov/publication/twri04A3.]

Hill, M.C., and Tiedeman, C.R., 2007, Effective groundwater model calibration, with analysis of data, sensitivities, predictions, and uncertainty: Hoboken, N.J., Wiley, 455 p.

Hirsch, R.M., and De Cicco, L.A., 2015, User guide to Exploration and Graphics for RivEr Trends (EGRET) and dataRetrieval: $\mathrm{R}$ packages for hydrologic data (version 2.0, February 2015): U.S. Geological Survey Techniques and Methods, book 4, chap. A10, 93 p. [Also available at http://dx.doi.org/10.3133/tm4A10.]

In-Situ Inc., 2017, Innovations in water monitoring spec Sheet: In-Situ Inc., 2 p.: Fort Collins, Colo., accessed July 23, 2018, at https://in-situ.com/wp-content/uploads/2014/11/ SS_LevelTROLL_Spec_Sheet_Dec2017.pdf.

Kirkham, R.M., and Ladwig, L.R., 1979, Coal resources of the Denver and Cheyenne basins, Colorado: Colorado Geological Survey Resource Series 5, 70 p., 5 pls.

Major, T.J., Robson, S.G., Romero, J.C., and Zawistowski, S., 1983, Hydrogeologic data from parts of the Denver Basin, Colorado: U.S. Geological Survey Open-File Report 83-274, 425 p. [Also available at https://pubs.er.usgs.gov/ publication/ofr83274.]

Maupin, M.A., Kenny, J.F., Hutson, S.S., Lovelace, J.K., Barber, N.L., and Linsey, K.S., 2014, Estimated use of water in the United States in 2010: U.S. Geological Survey Circular 1405, 56 p. [Also available at http://dx.doi.org/10.3133/cir1405.]

McConaghy, J.A., Chase, G.H., Boettcher, A.J., and Major, T.J., 1964, Hydrogeologic data of the Denver Basin, Colorado: Colorado Water Conservation Board, Basic-Data Report no. 15, 224 p.

Moore, J.E., Raynolds, R.G., and Dechesne, M., 2007, Bedrock aquifers and population growth in the Denver Basin, Colorado, USA: Episodes, v. 30, no. 2, p. 115-118.

National Geodetic Survey, 2018, OPUS: Online Positioning User Service: National Oceanic and Atmospheric Administration, accessed July and August 2017 and June 2018 at https://www.ngs.noaa.gov/OPUS/.
Paschke, S.S. ed., 2011, Groundwater availability of the Denver Basin aquifer system, Colorado: U.S. Geological Survey Professional Paper 1770, 274 p. [Also available at https://pubs.er.usgs.gov/publication/pp1770.]

R Core Team, 2018, R: A language and environment for statistical computing: Vienna, Austria, R Foundation for Statistical Computing, accessed August 1, 2018, at https://www.R-project.org/.

Raynolds, R.G., 2002, Upper Cretaceous and Tertiary stratigraphy of the Denver Basin, Colorado: Rocky Mountain Geology, v. 37, no. 2, p. 111-134.

Raynolds, R.G., 2004, Stratigraphy and water levels in the Arapahoe aquifer, Douglas County area, Denver Basin, Colorado: The Mountain Geologist, v. 41, no. 4, p. 195-210.

Raynolds, R.G., Johnson, K.R., Arnold, L.R., Farnham, T.M., Fleming, R.F., Hicks, J.F., Kelley, S.A., Lapey, L.A., Nichols, D.J., Obradovich, J.D., and Wilson, M.D., 2001, The Kiowa core-A continuous drill core through the Denver Basin bedrock aquifers at Kiowa, Elbert County, Colorado: U.S. Geological Survey Open-File Report 2001-185, 72 p. [Also available at https://pubs.er.usgs.gov/publication/ofr01185.]

Robson, S.G., 1987, Bedrock aquifers in the Denver Basin, Colorado-A quantitative water-resources appraisal: U.S. Geological Survey Professional Paper 1257, 73 p. [Also available at https://pubs.er.usgs.gov/publication/pp1257.]

Robson, S.G., Romero, J.C., and Zawistowski, S., 1981a, Geologic structure, hydrology, and water quality of the Arapahoe aquifer in the Denver Basin, Colorado: U.S. Geological Survey Hydrologic Investigations Atlas HA-646, 3 sheets, scale 1:500,000. [Also available at https://pubs.er.usgs.gov/publication/ha647.]

Robson, S.G., Wacinski, A., Zawistowski, S., and Romero, J.C., 1981b, Geologic structure, hydrology, and water quality of the Laramie-Fox Hills aquifer in the Denver Basin, Colorado: U.S. Geological Survey Hydrologic Investigations Atlas, HA-650, 3 sheets, scale 1:500,000. [Also available at https://pubs.er.usgs.gov/publication/ha650.]

Romero, J.C., 1976, Ground-water resources of the bedrock aquifers of the Denver Basin: Denver, Colo., Colorado Division of Water Resources Report, 109 p.

Rydlund, P.H., Jr., and Densmore, B.K., 2012, Methods of practice and guidelines for using survey-grade global navigation satellite systems (GNSS) to establish vertical datum in the United States Geological Survey: U.S. Geological Survey Techniques and Methods, book 11, chap. D1, 102 p. [Also available at http://pubs.usgs.gov/tm/11d1/.]

Schneider, P.A., Jr., 1980, Water-supply assessment of the Laramie-Fox Hills aquifer in parts of Adams, Boulder, Jefferson, and Weld Counties, Colorado: U.S. Geological Survey Open-File Report 80-327, 21 p. [Also available at https://pubs.er.usgs.gov/publication/ofr80327.] 
Tonkin, M.J., Tiedeman, C.R., Ely, D.M., and Hill, M.C., 2007, OPR-PPR, a computer program for assessing data importance to model predictions using linear statistics: U.S. Geological Survey Techniques and Methods 6-E2, 115 p. [Also available at https://pubs.er.usgs.gov/publication/tm6E2.]

U.S. Census Bureau, 1996, Population of States and Counties of the United States: 1790 to 1990: Suitland, Md., U.S.

Census Bureau, 225 p.

U.S. Census Bureau, 2000, Census 2000 Summary File 1: Suitland, Md., U.S. Census Bureau, accessed July 20, 2018 at https://www.census.gov/census2000/sumfile1.html.

U.S. Census Bureau, 2017, Annual Estimates of Resident Population: April 1, 2010 to July 1, 2017, Population Division: Suitland, Md., accessed July 20, 2018, at https:// www.census.gov/quickfacts/fact/table/elbertcountycolorado/ PST045217\#viewtop.

Wireman, M., and Romero, J.C., 1989, Bibliography of geology and ground water geology for the Denver Basin, Colorado: Denver, Colo., Colorado Division of Water Resources Office of the State Engineer, $12 \mathrm{p}$.

Woodard L.L., Sanford, W., Raynolds, R.G., 2002, Stratigraphic variability of specific yield within bedrock aquifers of the Denver Basin, Colorado: Rocky Mountain Geology, v. 37, no. 2, p. 229-236.

Publishing support provided by the Science Publishing Network, Denver Publishing Service Center

For more information concerning the research in this report, contact the Director, USGS Colorado Water Science Center

Box 25046, Mail Stop 415

Denver, CO 80225

(303) 236-4882

Or visit the Colorado Water Science Center website at

https://co.water.usgs.gov/ 


\section{Appendix 1. Groundwater-Well Measurement Diagram}

A

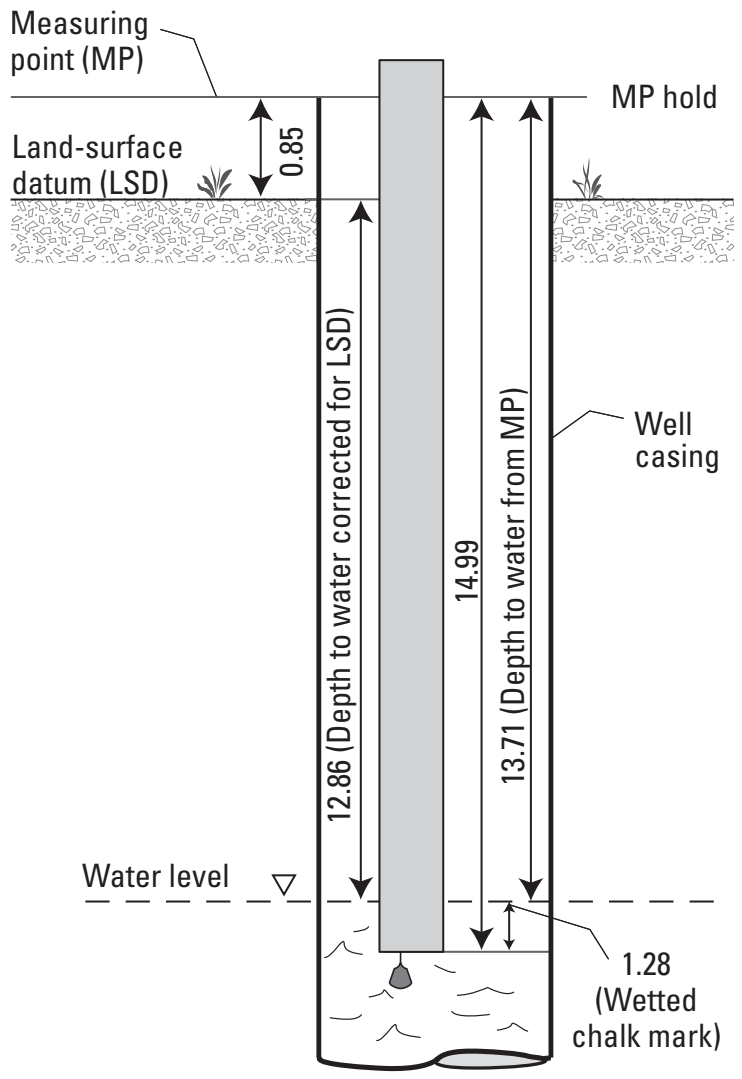

$\boldsymbol{B}$

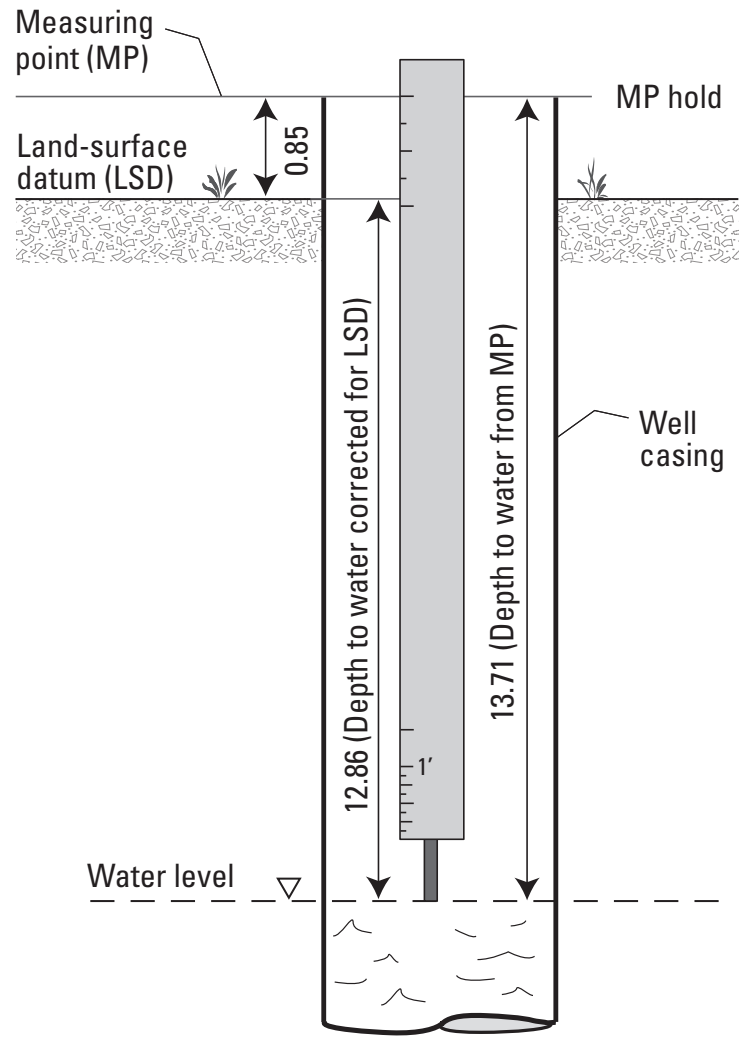

Figure 1.1. Diagram showing example measurement point and groundwater-level measurement using $A$, a calibrated steel tape with chalk, and $B$, a calibrated electrical tape. Modified from Cunningham and Schalk, 2011. (Values are in feet) 


\section{Appendix 2. Hydrographs Showing Groundwater-Level Elevation Through Time for Wells in the Elbert County Groundwater-Level Monitoring Network.}

Hydrographs showing groundwater-level elevation through time for each well in this study are presented in this appendix. Measurement periods vary but are generally from April 2015 to June 2018 for discrete measurements and summer/fall 2015 to June 2018 for continuous measurements. Daily maximum groundwater-level elevation, in feet above North American Vertical Datum of 1988, is plotted for continuous measurements. Daily median and minimum values were not plotted, but data are available; see the "Accessing Data" section. Discrete measurement symbols vary by status; see "Study Methods" section for a description of the status codes. UDAW, upper Dawson aquifer; LDAW, lower Dawson aquifer; DENV, Denver aquifer; ARAP, Arapahoe aquifer; LARA, Laramie-Fox Hills aquifer.

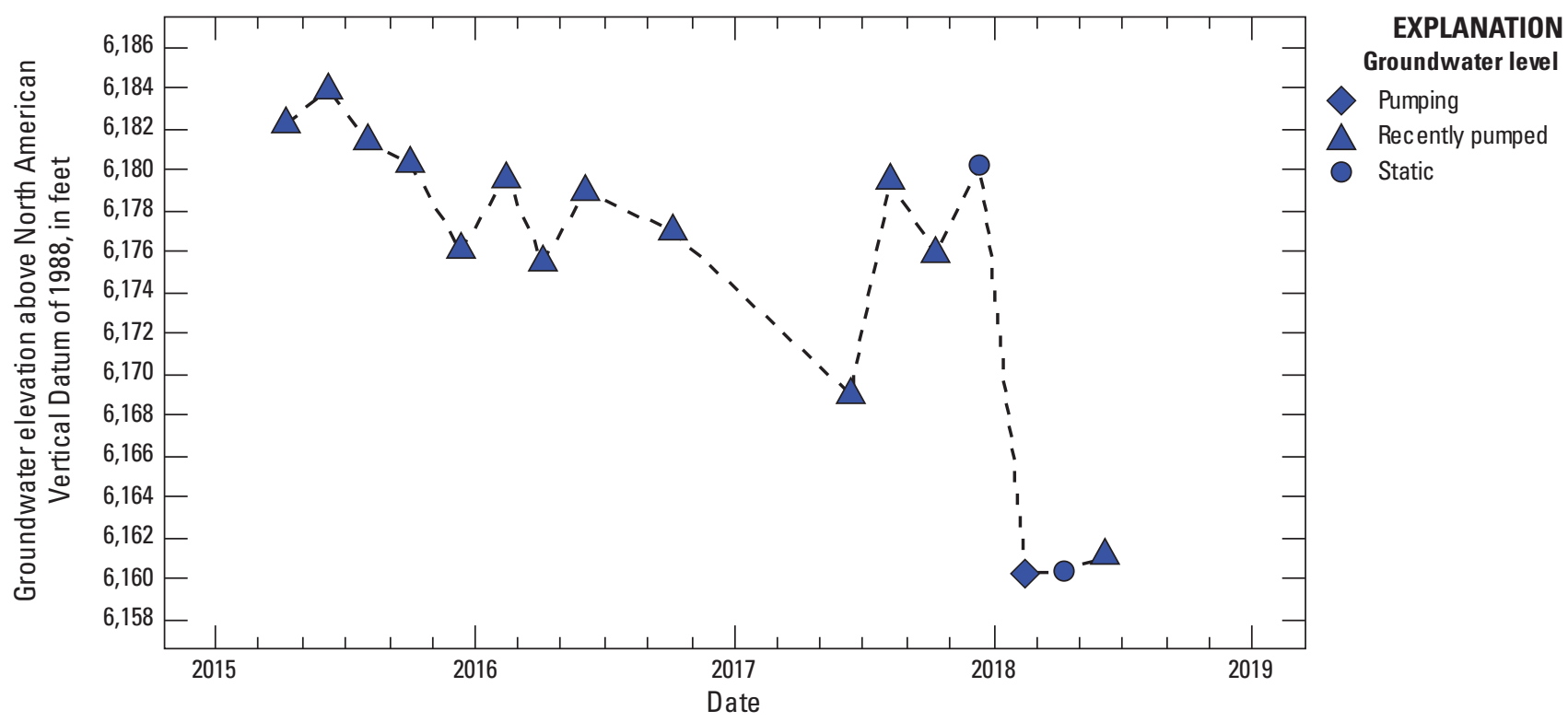

Figure 2.1. Groundwater-level hydrograph for DENV 17, USGS site number 390755104172501 , Elbert County, Colorado.

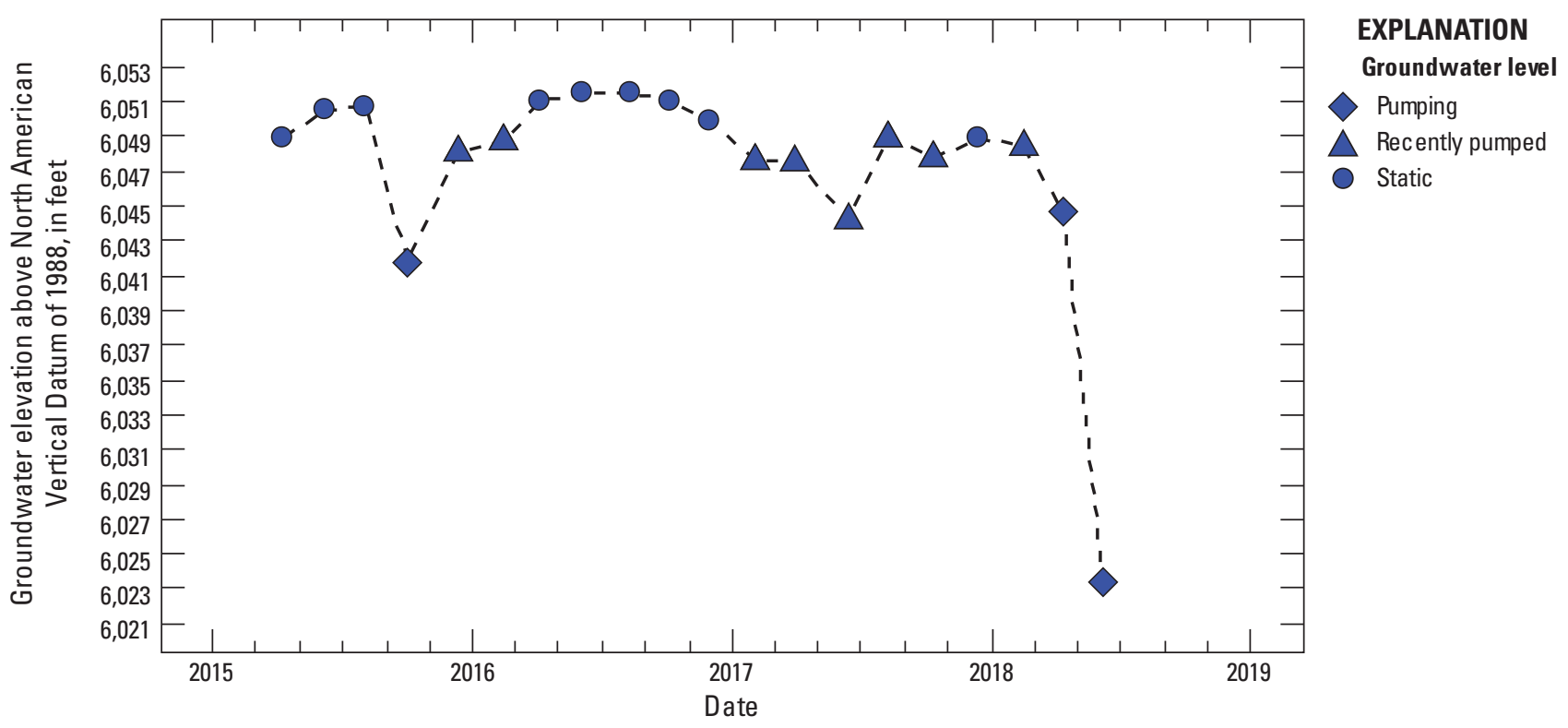

Figure 2.2. Groundwater-level hydrograph for ARAP 8, USGS site number 390800104172601, Elbert County, Colorado. 


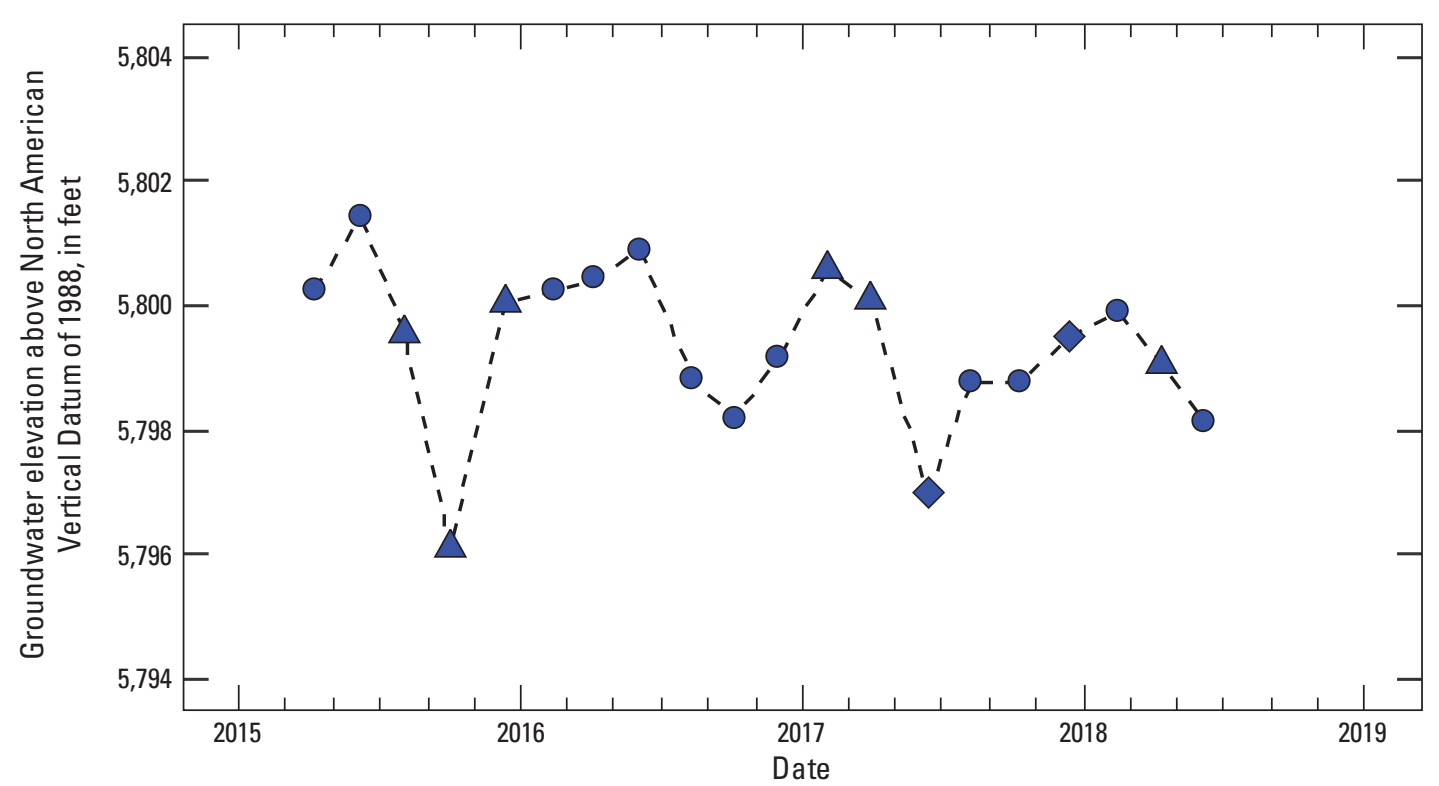

EXPLANATION

Groundwater level

Pumping

$\triangle$ Rec ently pumped

Static

Figure 2.3. Groundwater-level hydrograph for LARA 7, USGS site number 390817104040301 , Elbert County, Colorado.

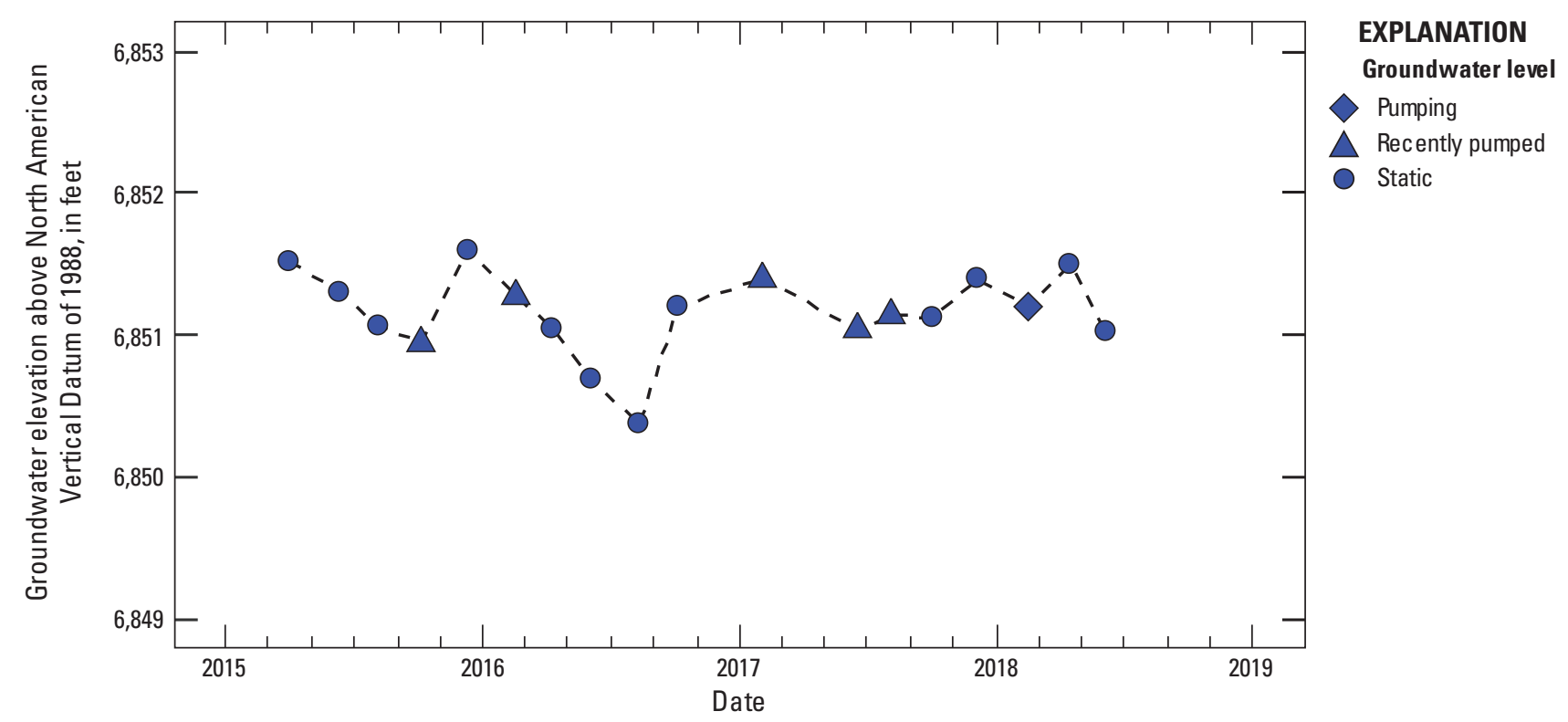

Figure 2.4. Groundwater-level hydrograph for DAWMAS26, USGS site number 390935104301001, Elbert County, Colorado. 


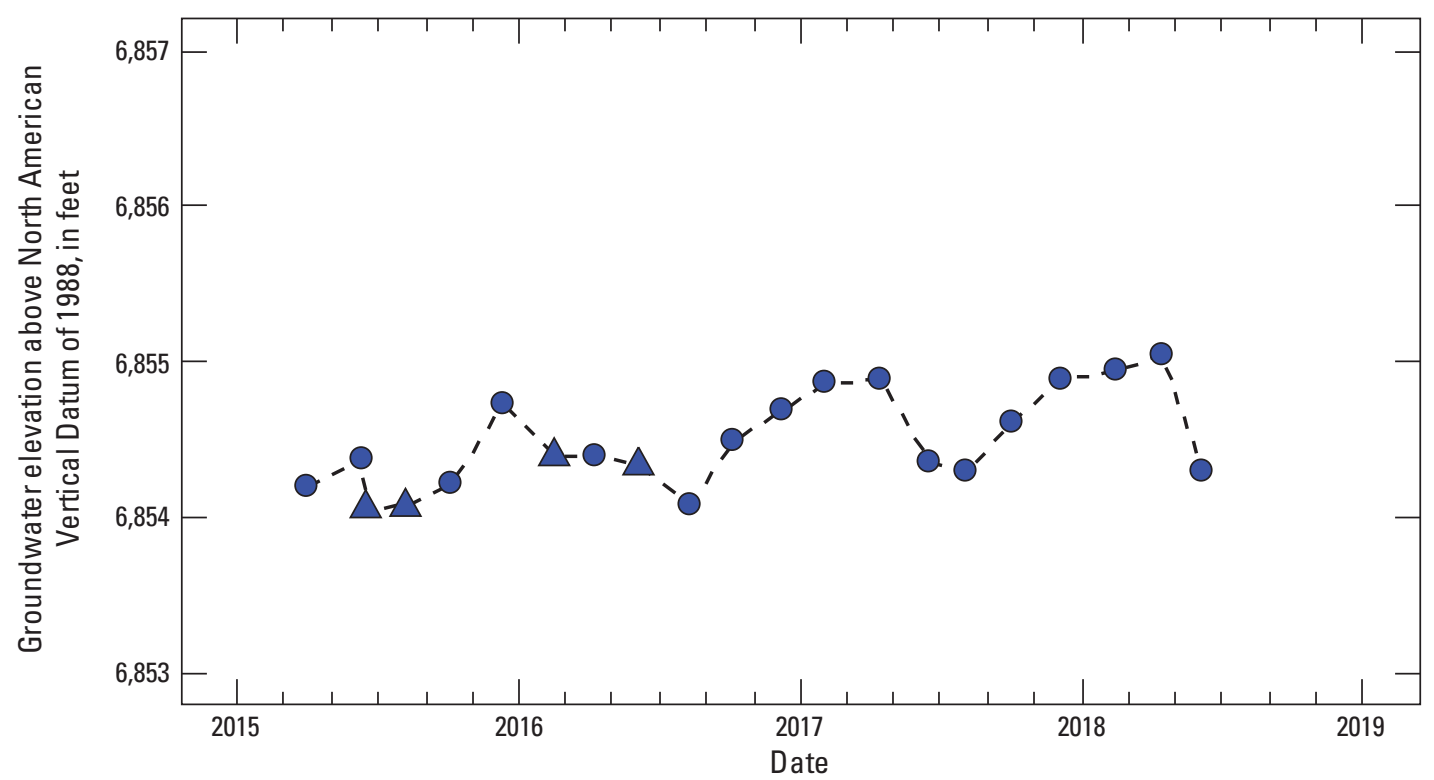

EXPLANATION

Groundwater level

$\triangle$ Recently pumped

Static

Figure 2.5. Groundwater-level hydrograph for UDAW 19, USGS site number 391126104354701 , Elbert County, Colorado.

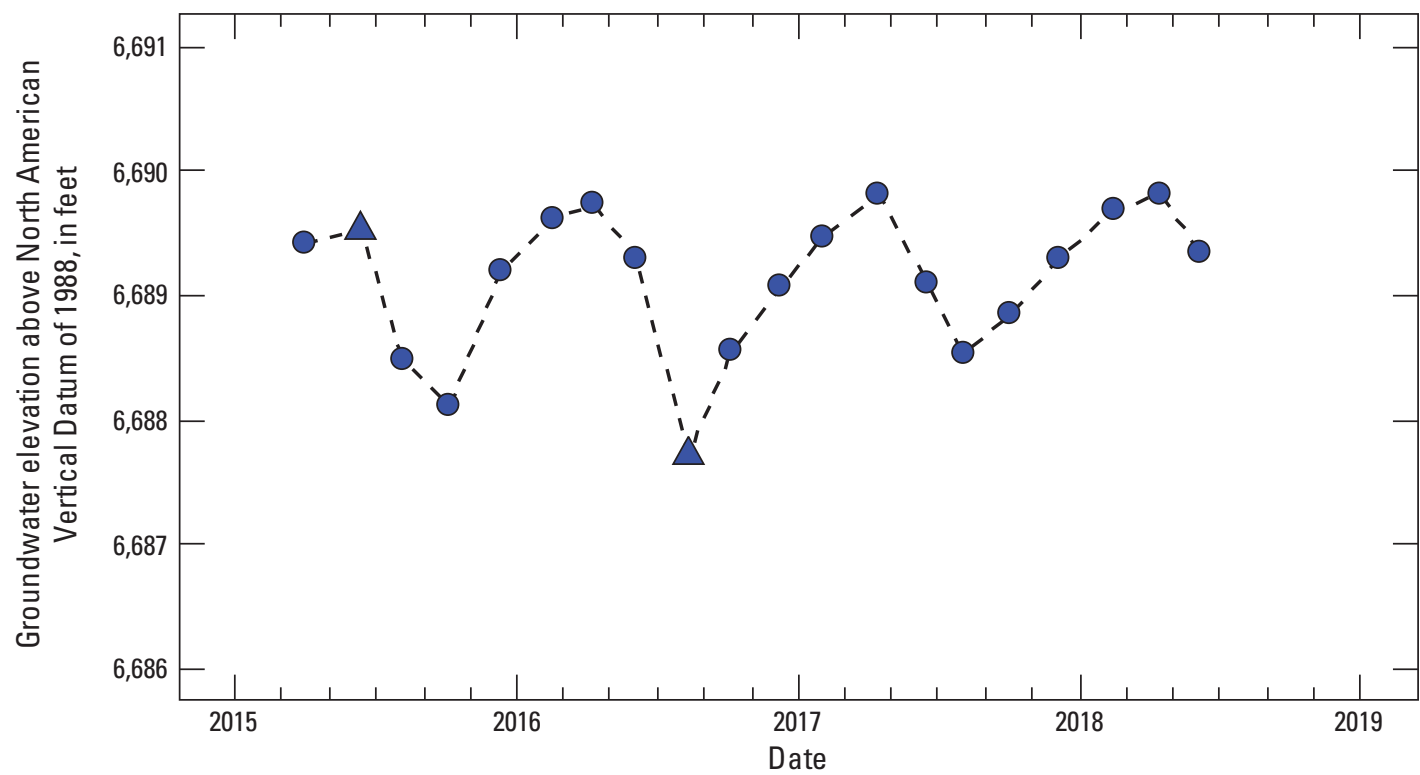

Rec ently pumped

Static

Figure 2.6. Groundwater-level hydrograph for DAWMAS27, USGS site number 391148104294101, Elbert County, Colorado. 


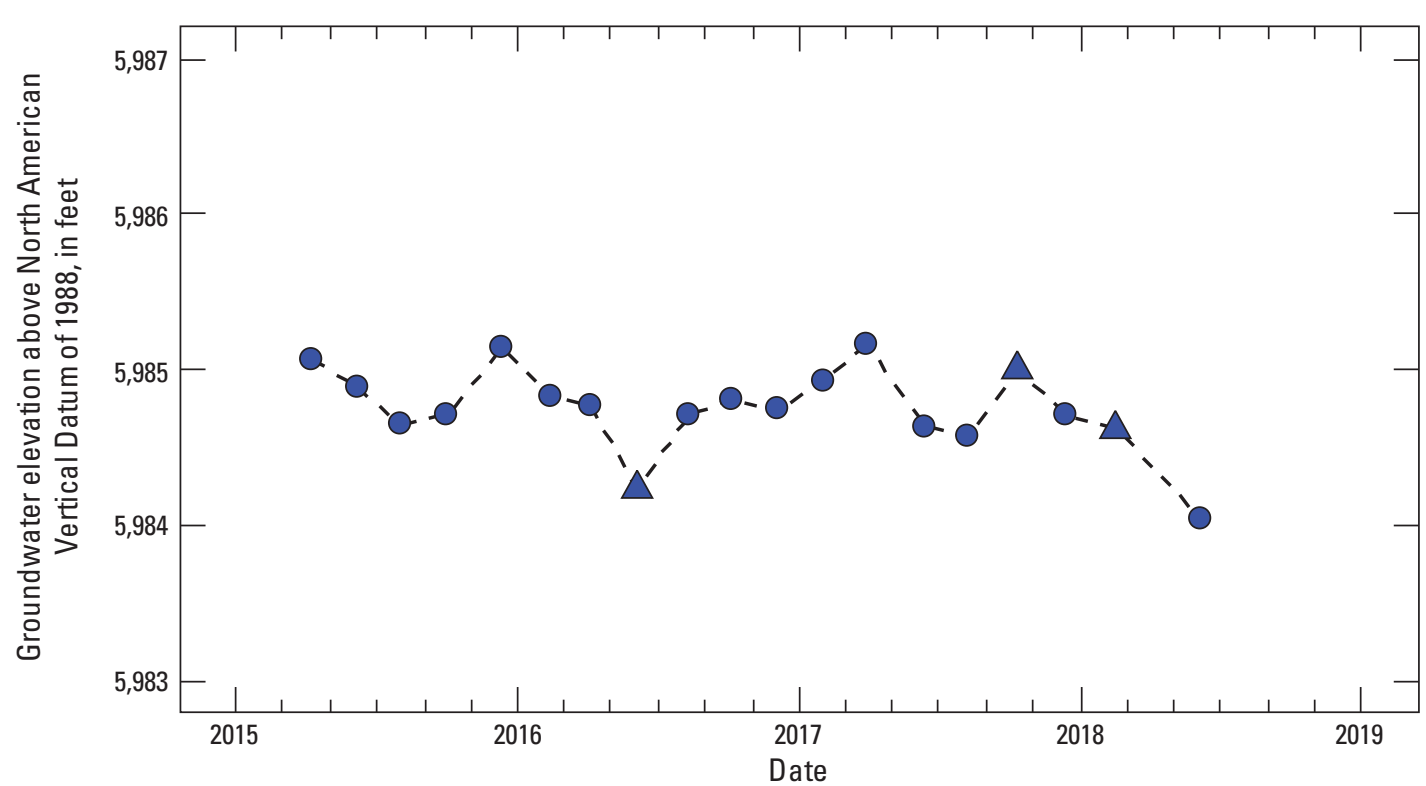

EXPLANATION

Groundwater level

$\triangle$ Recently pumped

- Static

Figure 2.7. Groundwater-level hydrograph for ARAP 7, USGS site number 391208104053301, Elbert County, Colorado.

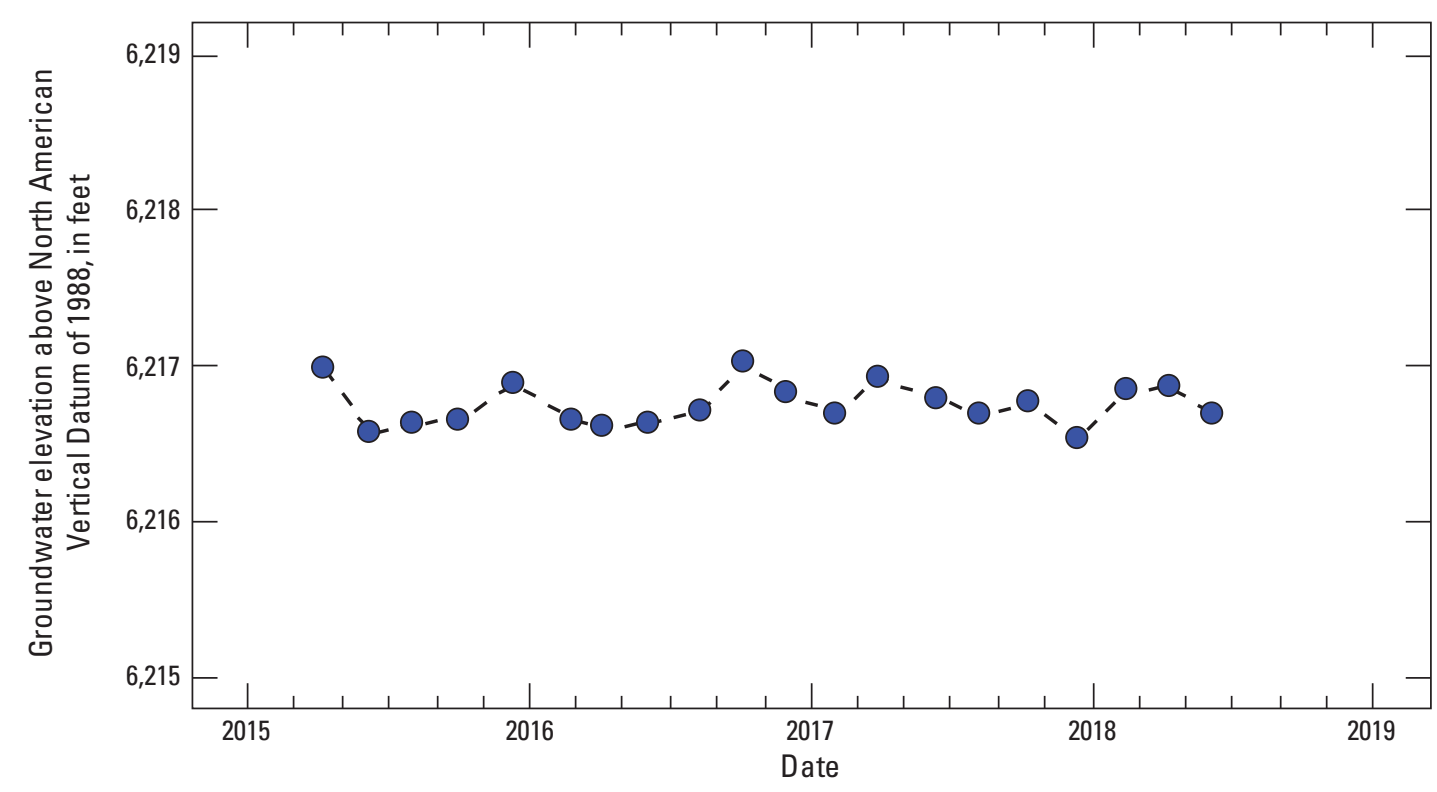

EXPLANATION

Groundwater level status

- Static

Figure 2.8. Groundwater-level hydrograph for DENV 16, USGS site number 391257104173601, Elbert County, Colorado. 


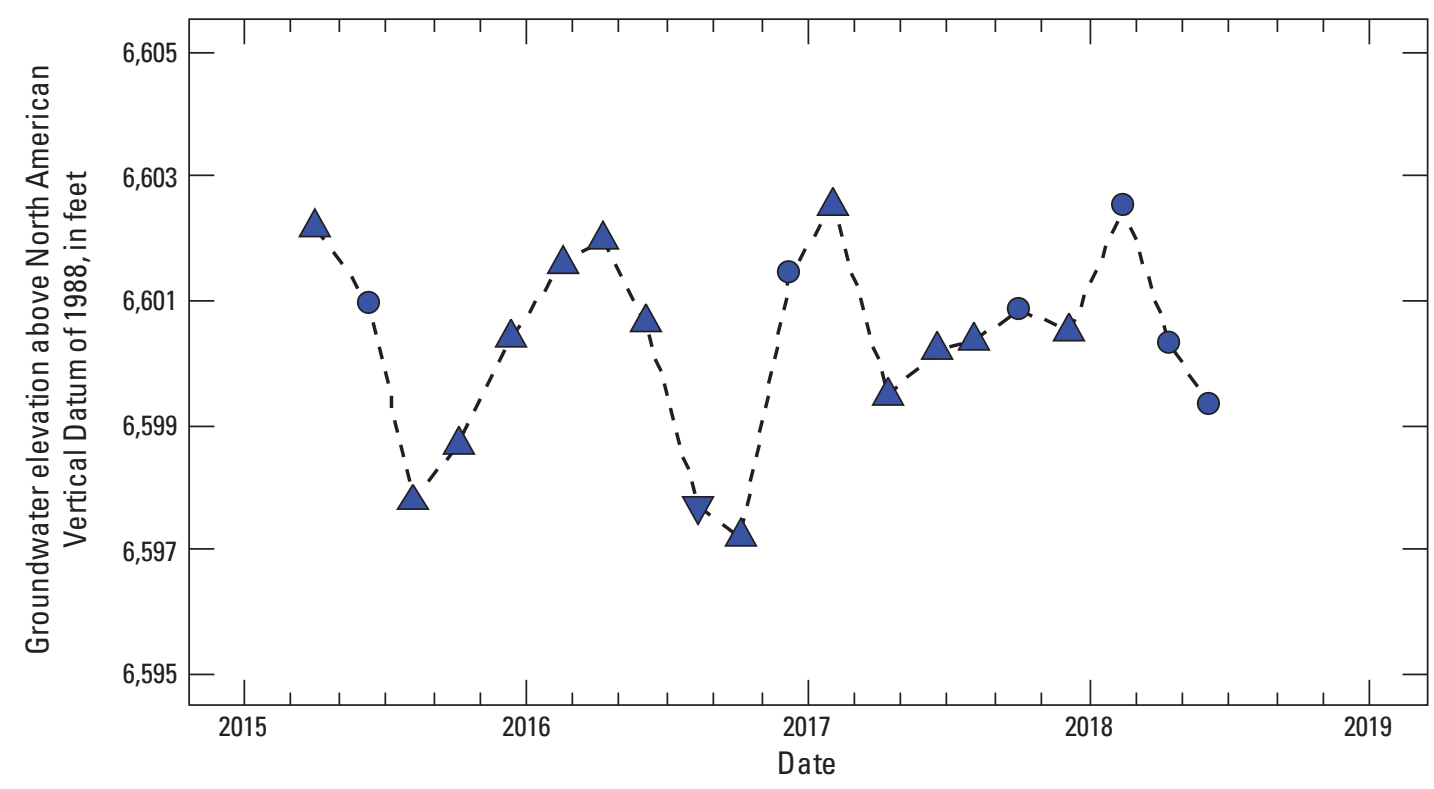

EXPLANATION

Groundwater level

$\nabla$ Nearby pumping

$\triangle$ Rec ently pumped

- Static

Figure 2.9. Groundwater-level hydrograph for LDAW 16, USGS site number 391502104273601, Elbert County, Colorado.

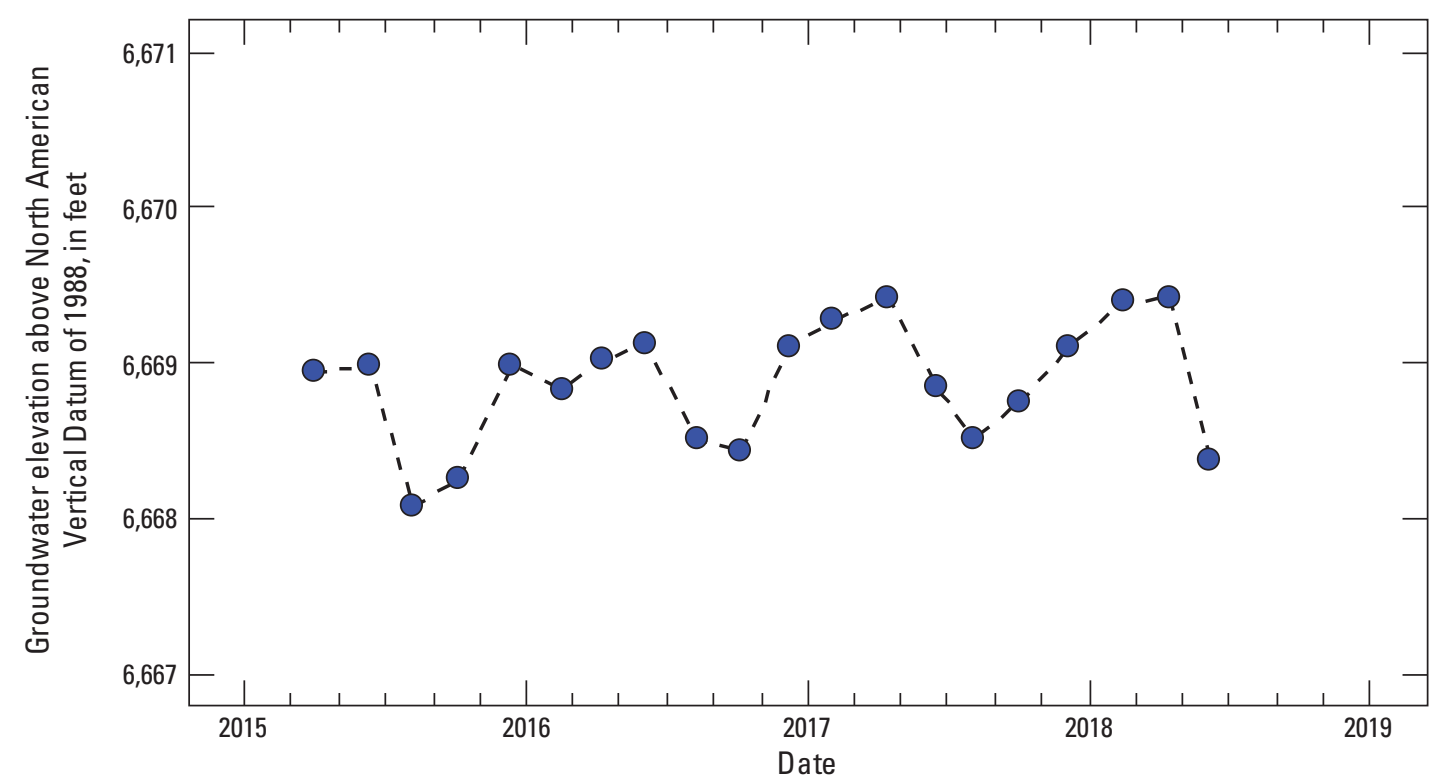

EXPLANATION

Groundwater level

- Static

Figure 2.10. Groundwater-level hydrograph for DAWMAS22, USGS site number 391545104335401, Elbert County, Colorado. 


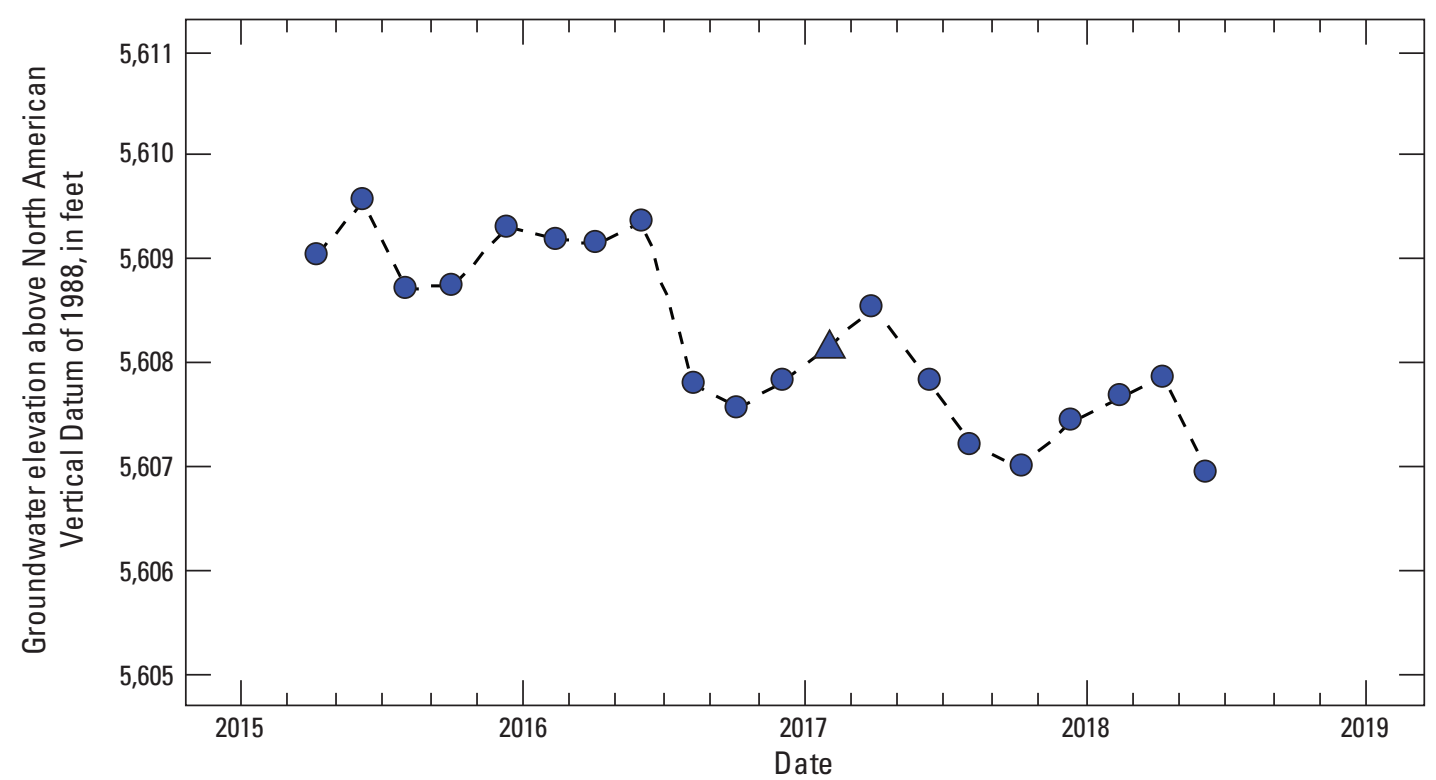

EXPLANATION

Groundwater level

$\triangle$ Rec ently pumped

Static

Figure 2.11. Groundwater-level hydrograph for LARA 6, USGS site number 391609104014001, Elbert County, Colorado.

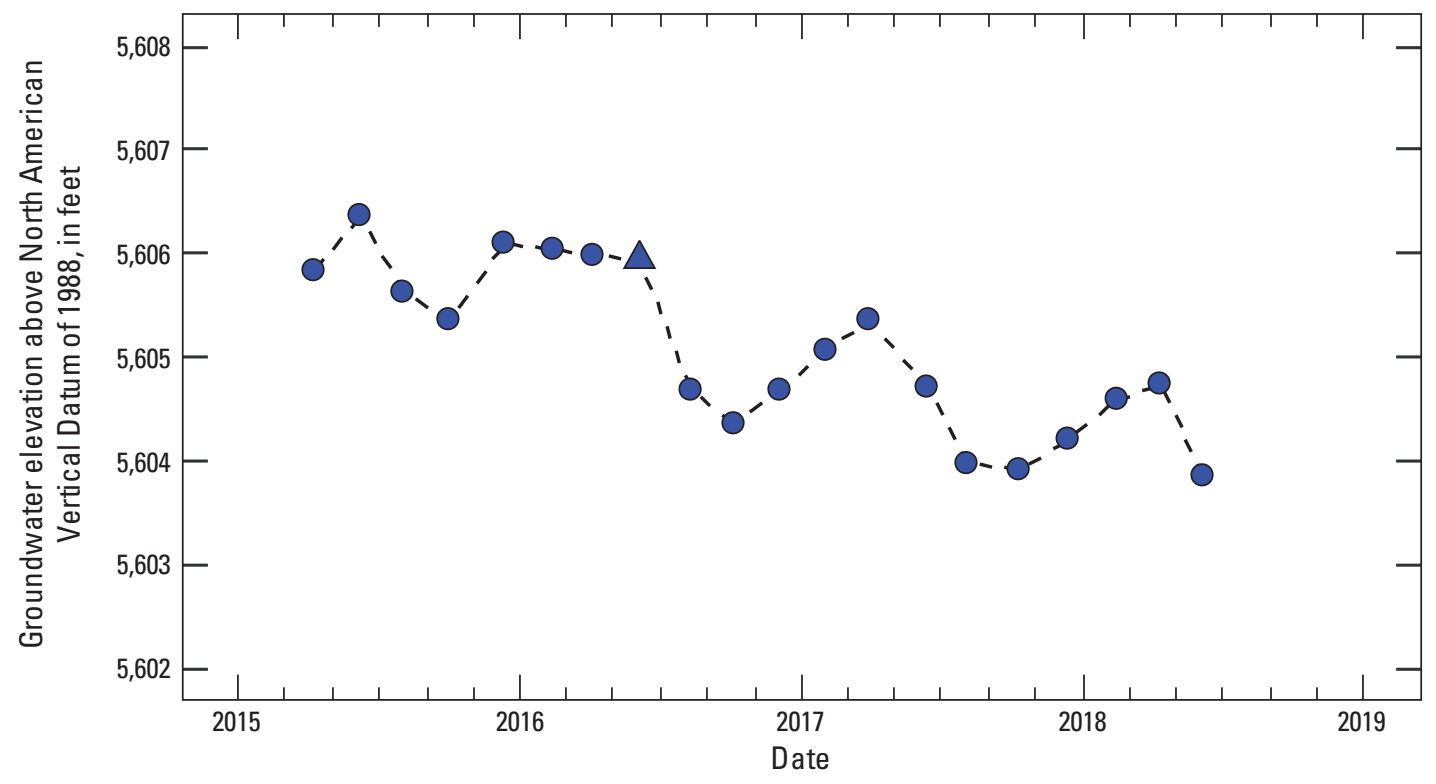

Figure 2.12. Groundwater-level hydrograph for LARA 5, USGS site number 391621104012001, Elbert County, Colorado. 


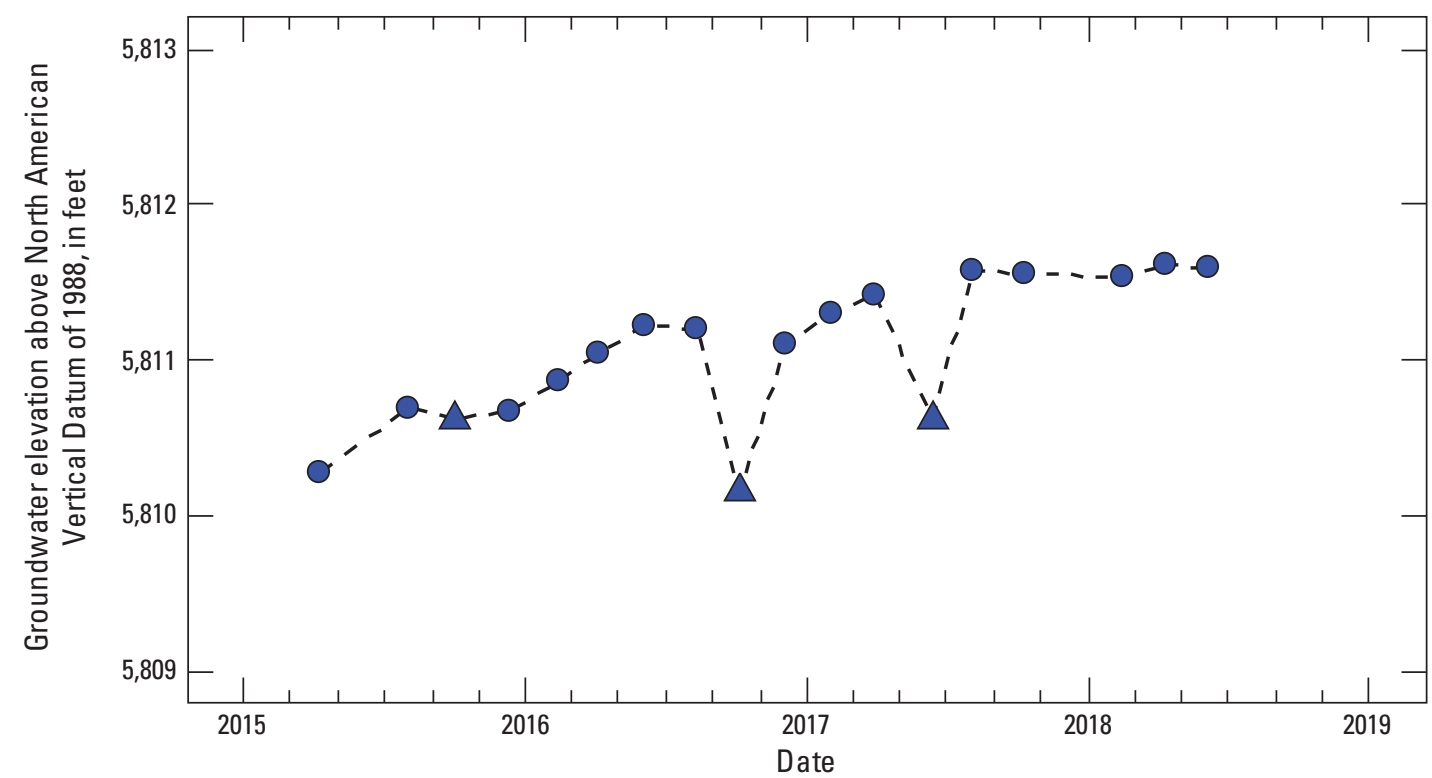

\section{EXPLANATION}

Groundwater level

$\triangle$ Recently pumped

- Static

Figure 2.13. Groundwater-level hydrograph for ARAPMAS27, USGS site number 391740104072401 , Elbert County, Colorado.

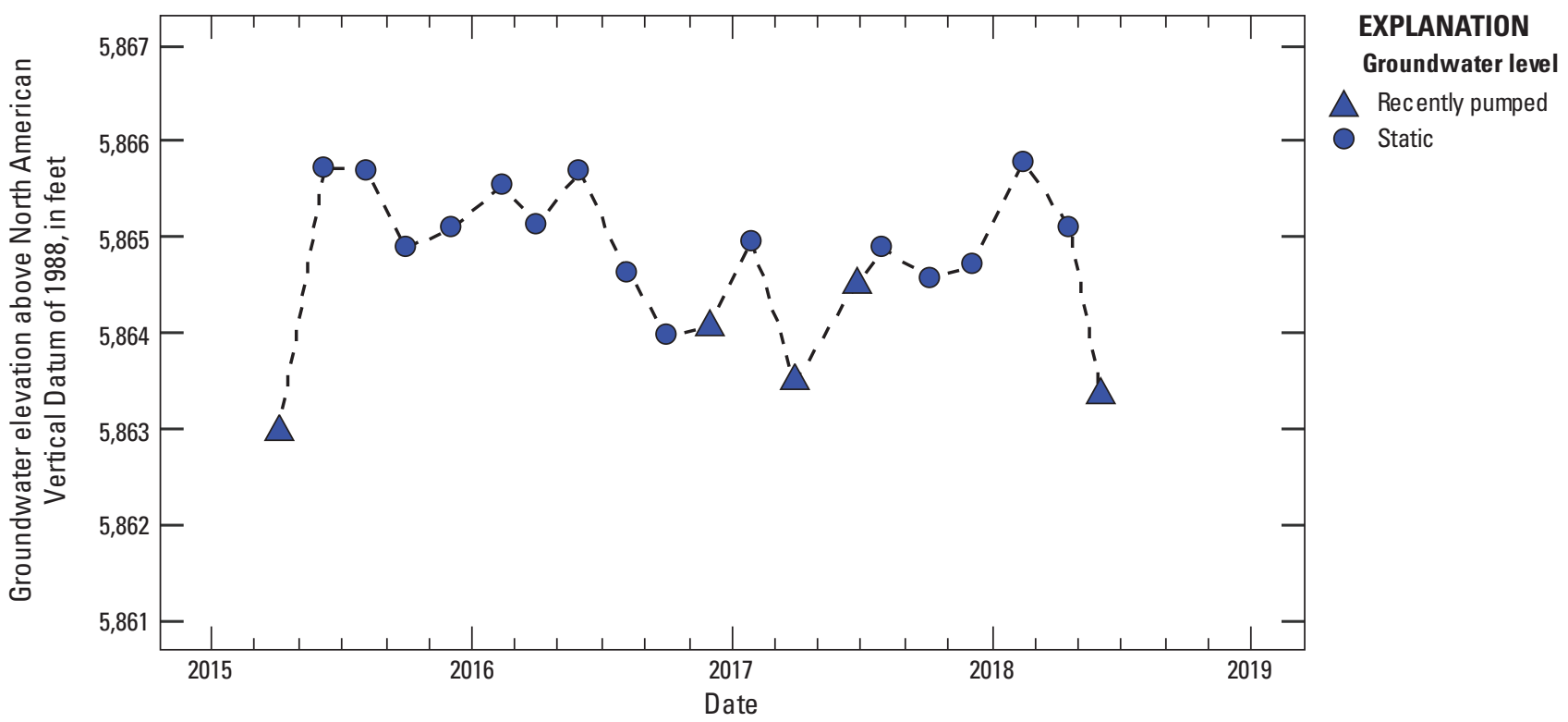

Figure 2.14. Groundwater-level hydrograph for DENV 15, USGS site number 391811104140301, Elbert County, Colorado. 


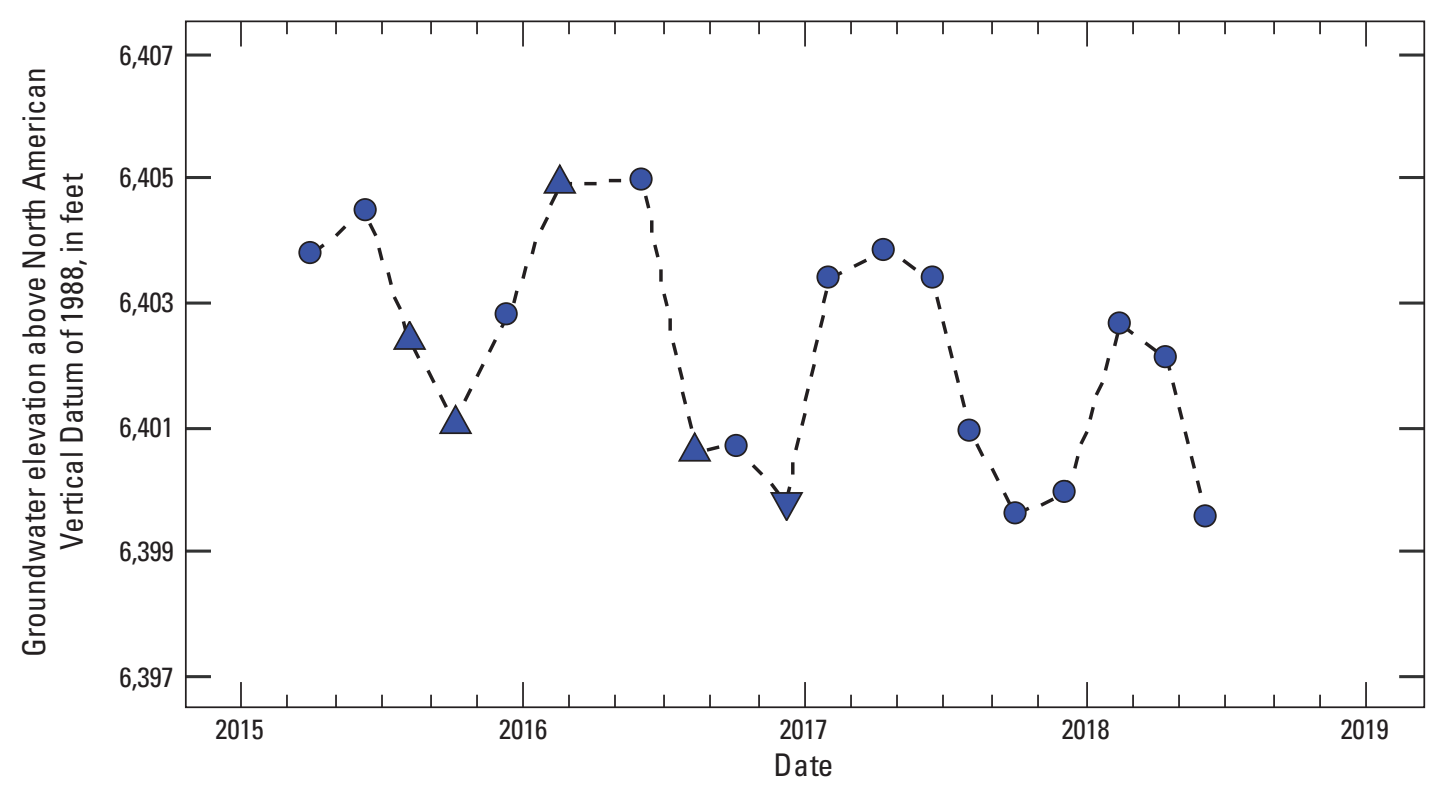

EXPLANATION

Groundwater level

$\nabla$ Nearby pumping

$\triangle$ Rec ently pumped

Static

Figure 2.15. Groundwater-level hydrograph for DENV 14, USGS site number 391821104270601, Elbert County, Colorado.

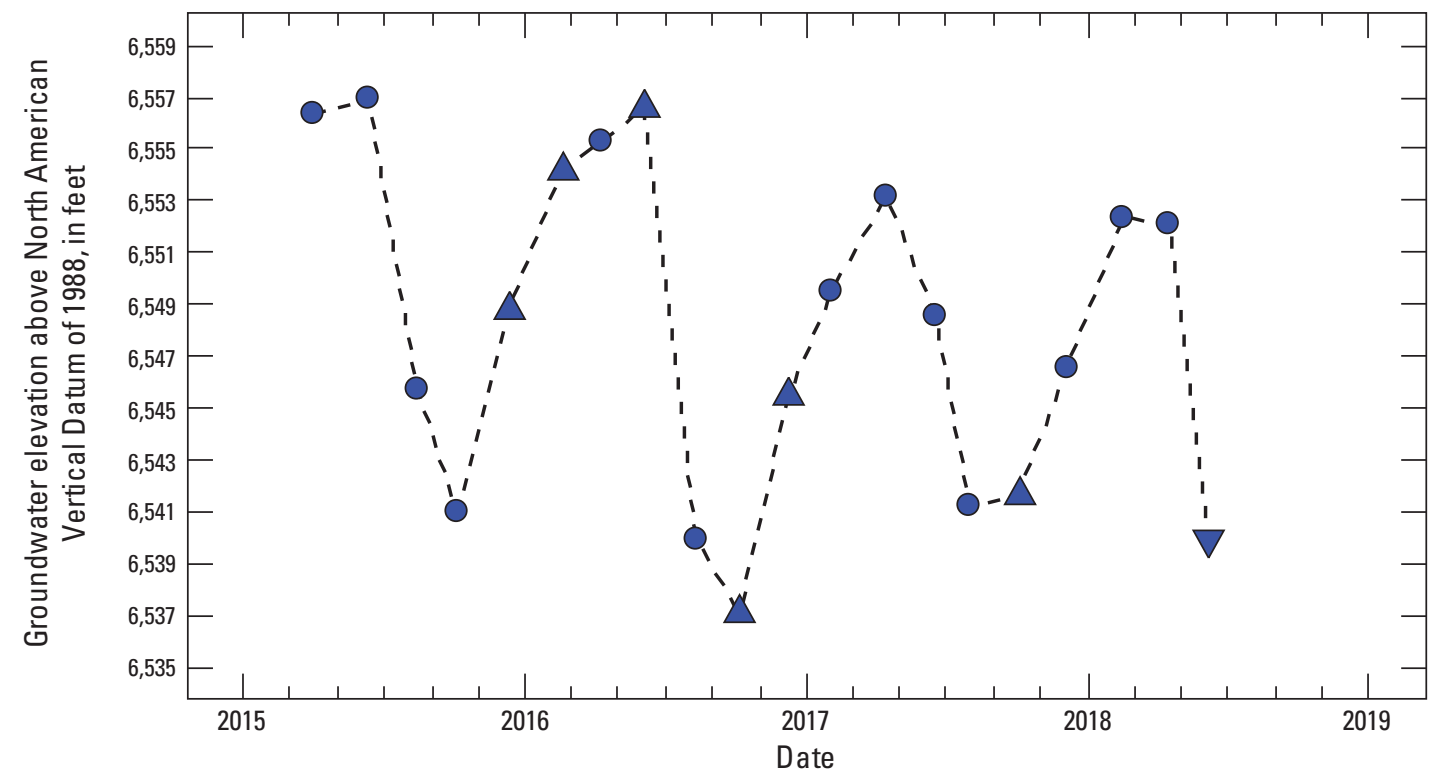

EXPLANATION

Groundwater level

$\checkmark$ Nearby pumping

$\triangle$ Recently pumped

- Static

Figure 2.16. Groundwater-level hydrograph for LDAW 15, USGS site number 391829104385301 , Elbert County, Colorado. 


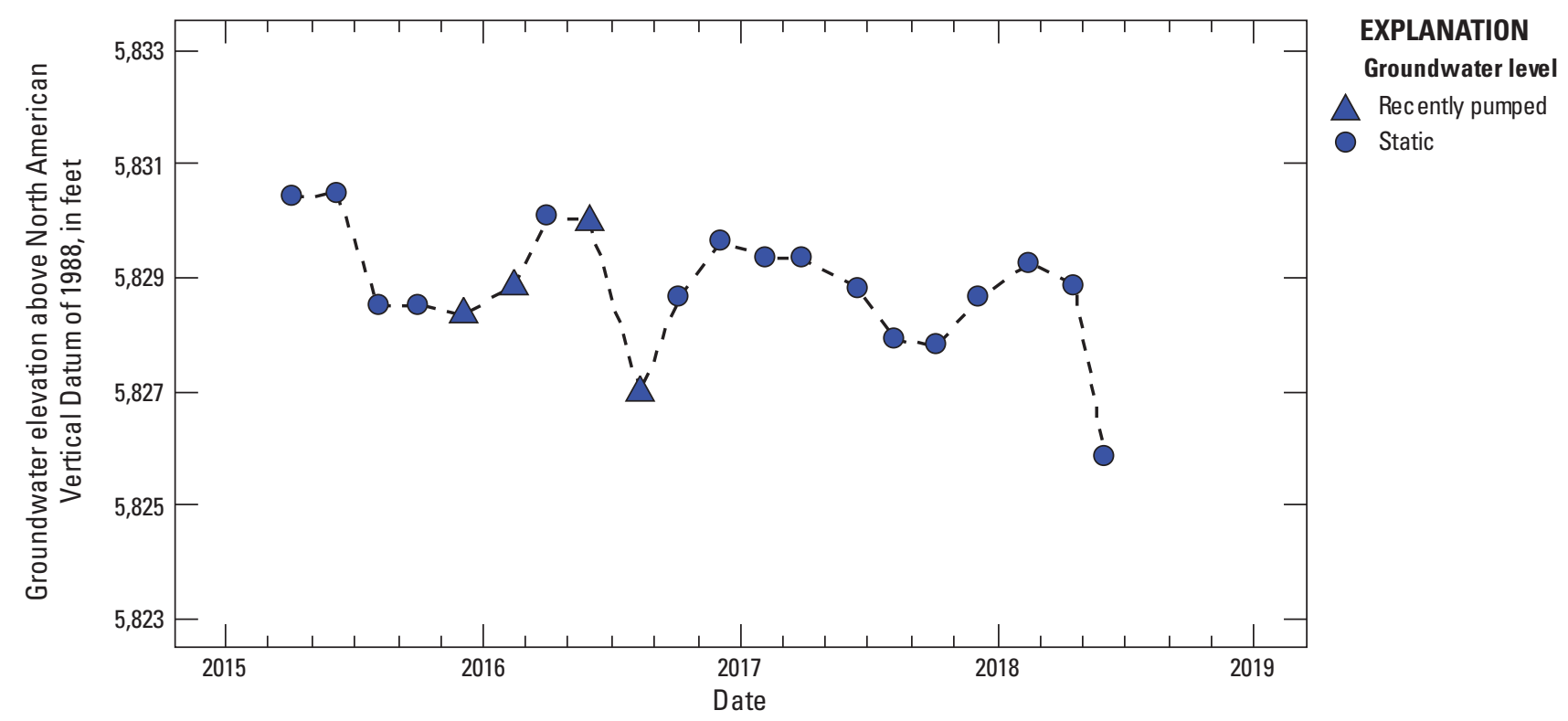

Figure 2.17. Groundwater-level hydrograph for ARAPMAS22, USGS site number 391834104205601, Elbert County, Colorado.

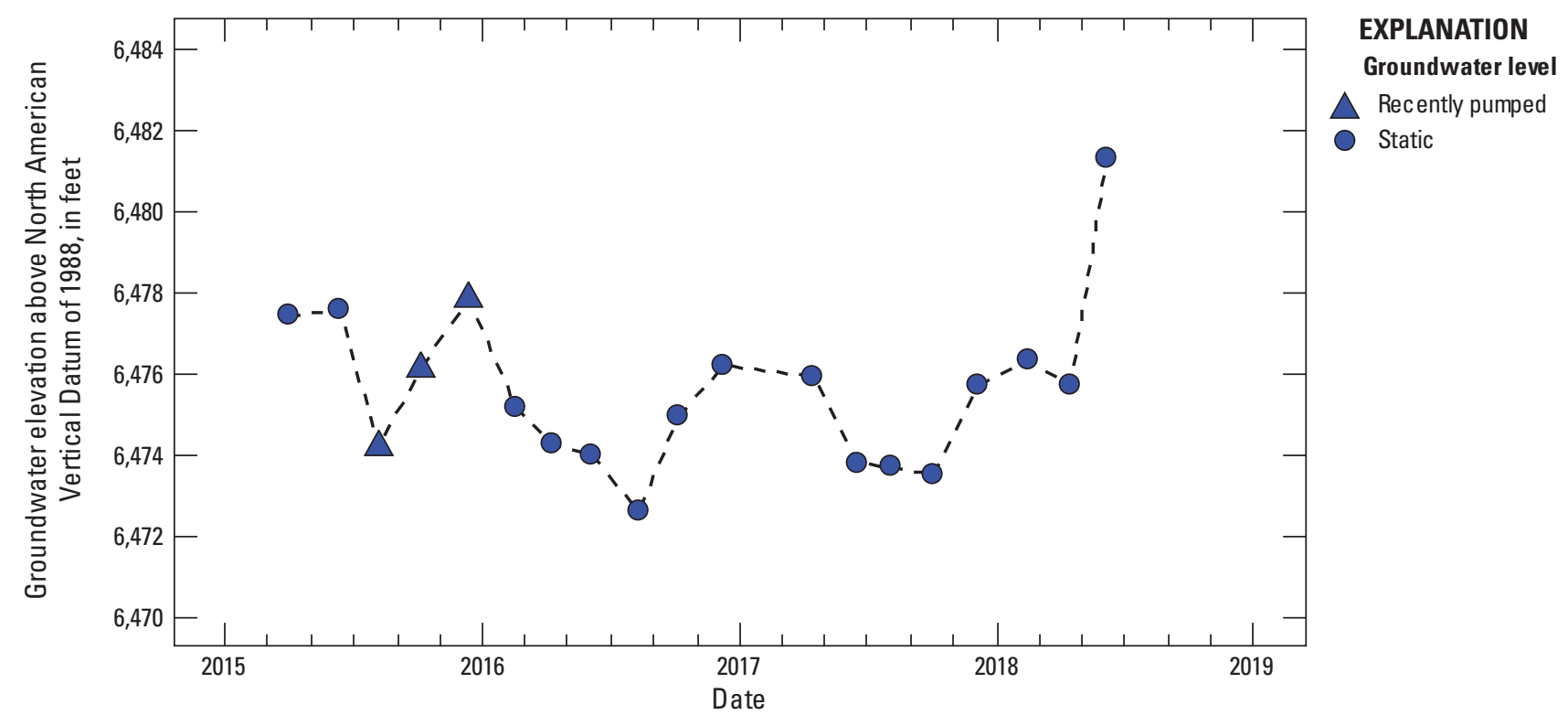

Figure 2.18. Groundwater-level hydrograph for DAWMAS28, USGS site number 391848104261401, Elbert County, Colorado. 


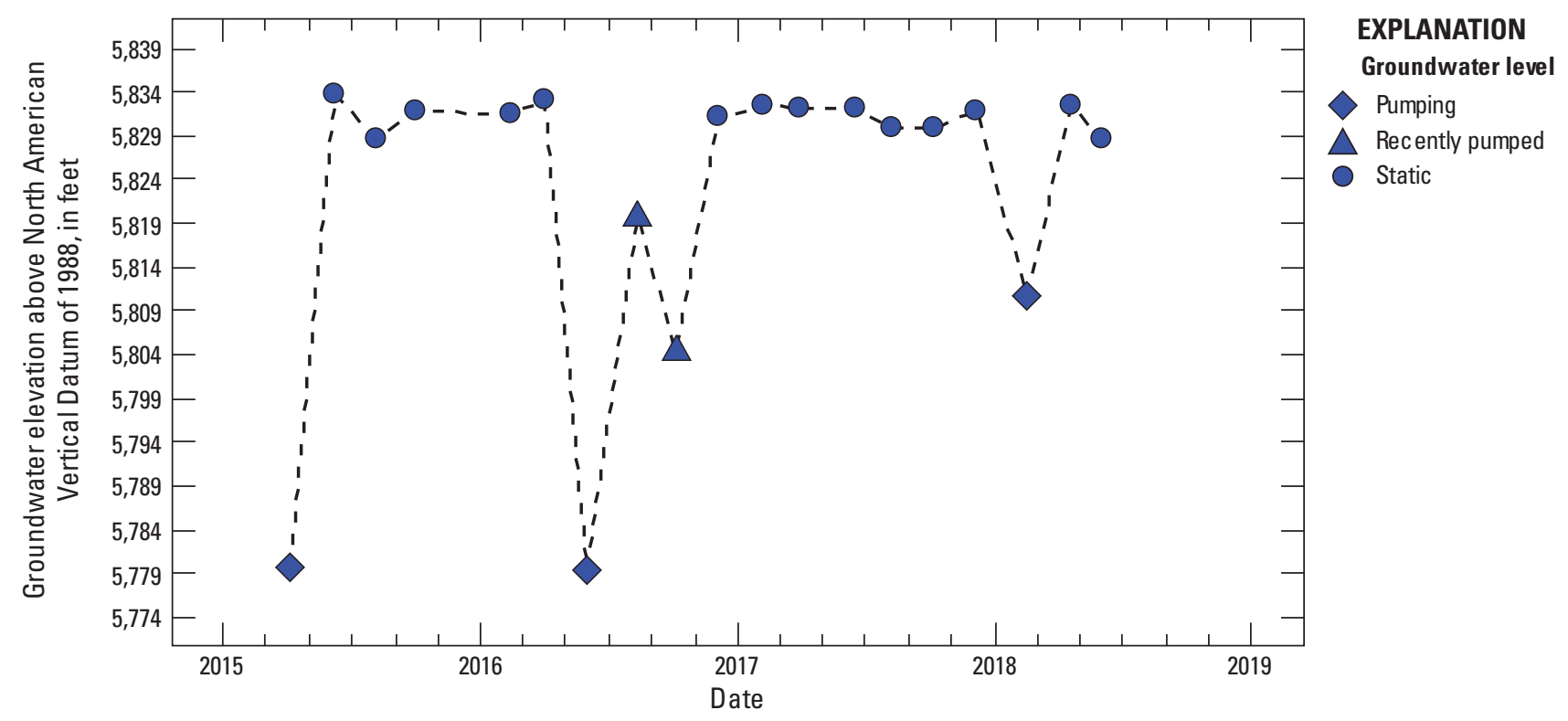

Figure 2.19. Groundwater-level hydrograph for DENMAS05, USGS site number 391851104204501, Elbert County, Colorado.

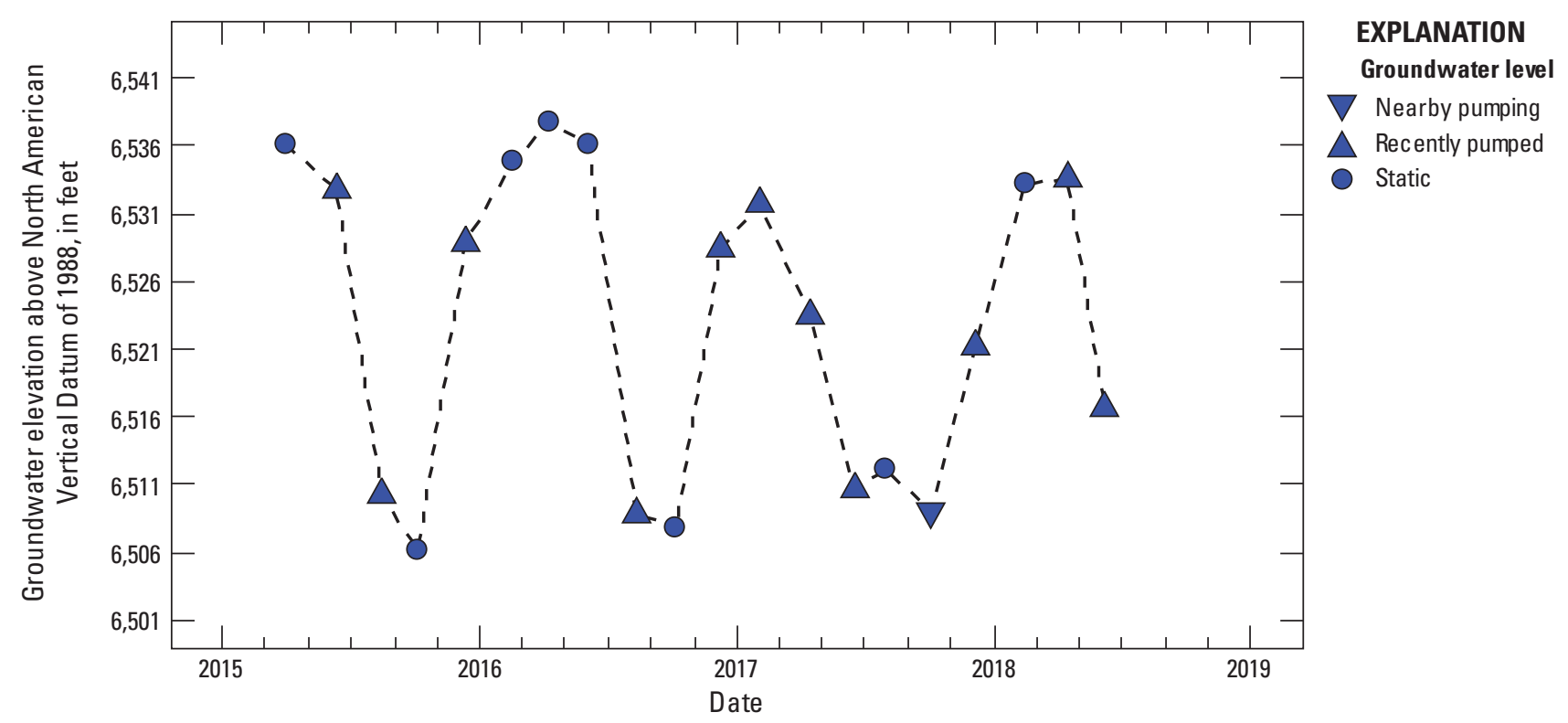

Figure 2.20. Groundwater-level hydrograph for DAWMAS16, USGS site number 391852104391301 , Elbert County, Colorado. 


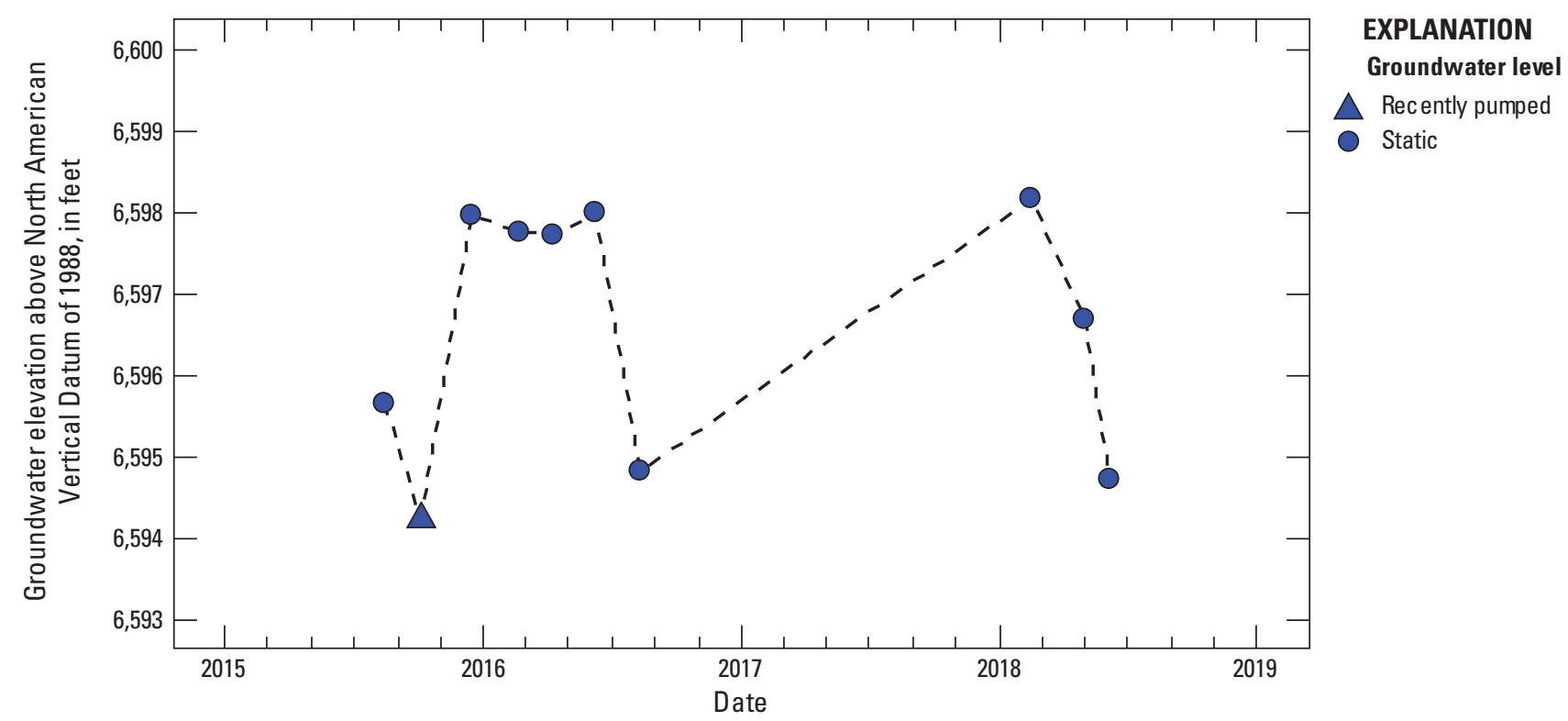

Figure 2.21. Groundwater-level hydrograph for UDAW 18, USGS site number 391915104375001, Elbert County, Colorado.

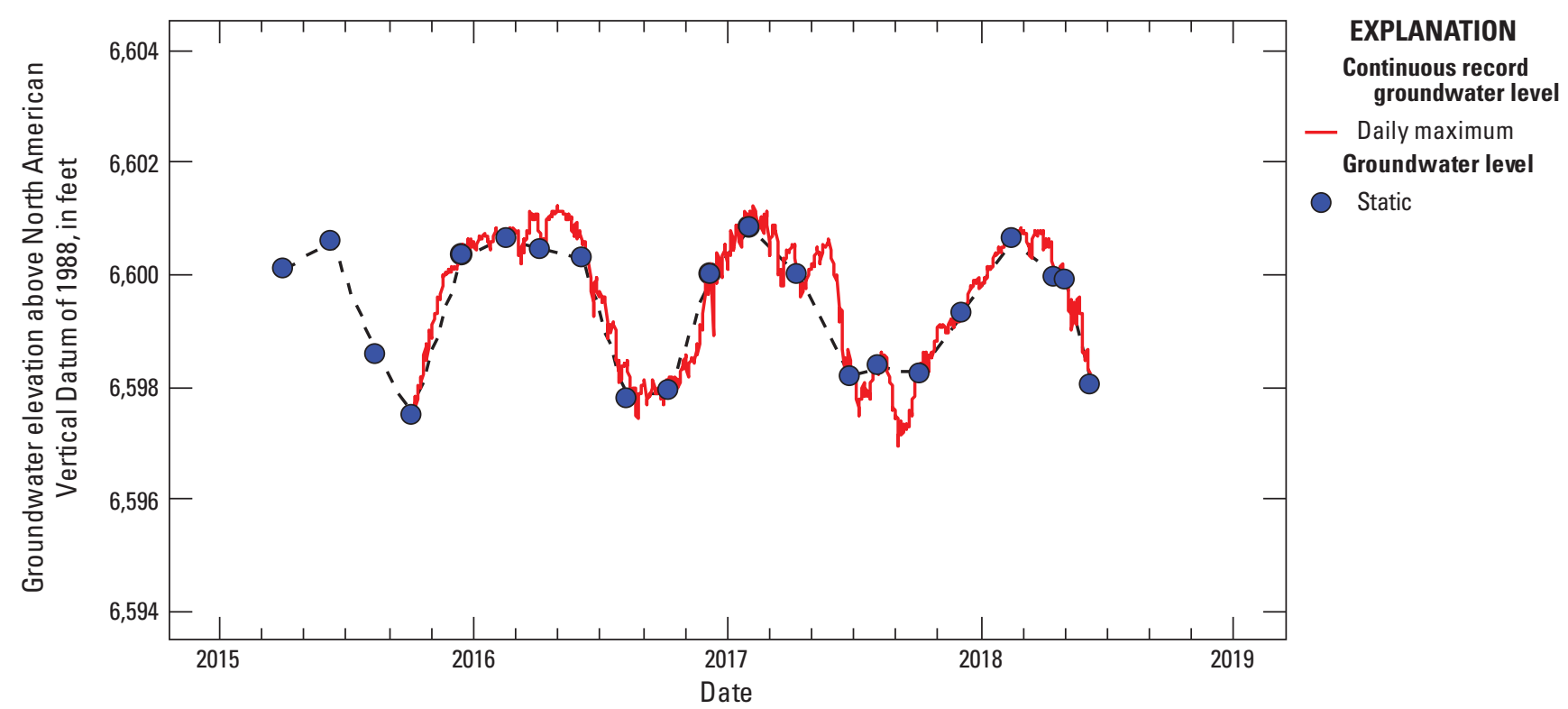

Figure 2.22. Groundwater-level hydrograph for UDAW 14, USGS site number 391924104374101, Elbert County, Colorado. 


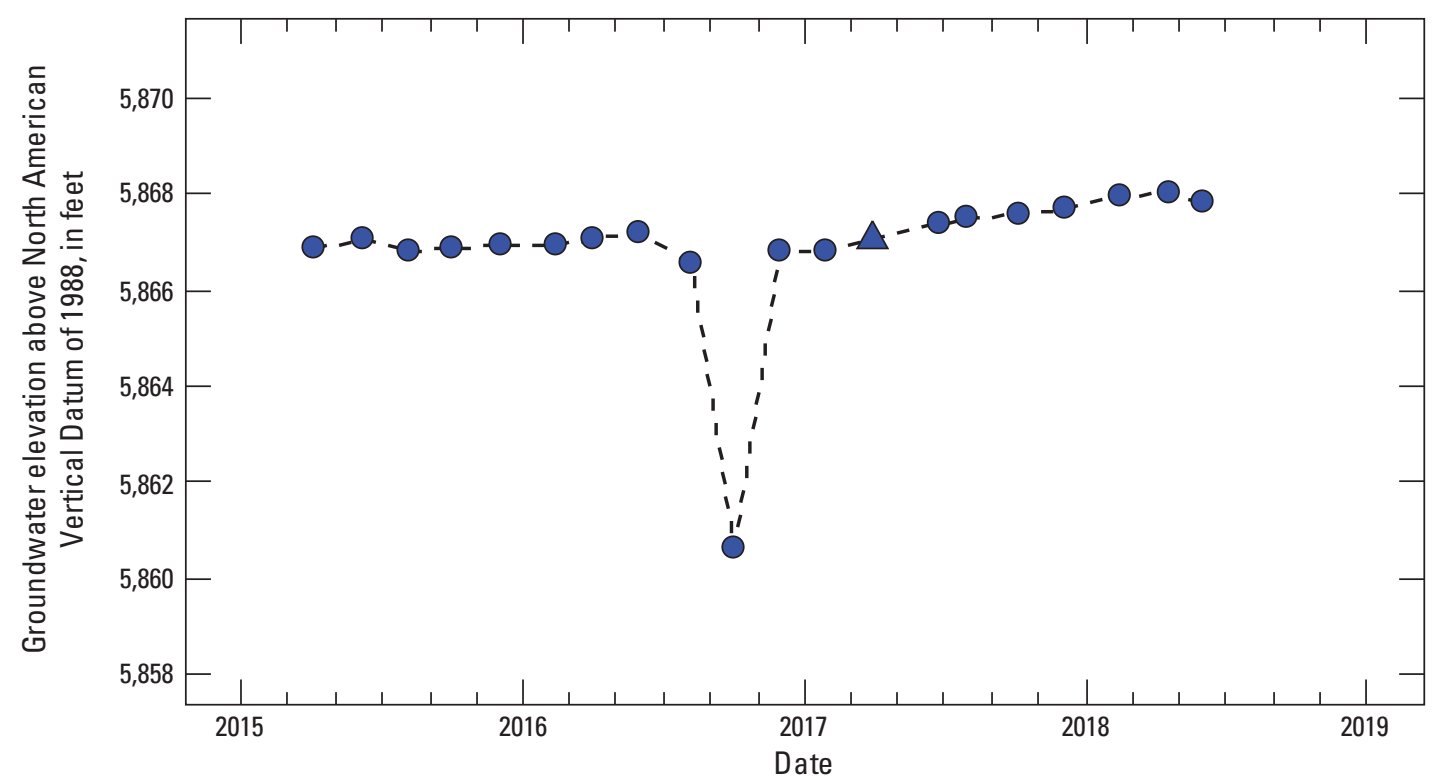

EXPLANATION

Groundwater level

$\triangle$ Recently pumped

- Static

Figure 2.23. Groundwater-level hydrograph for ARAP 6, USGS site number 391946104114501, Elbert County, Colorado.

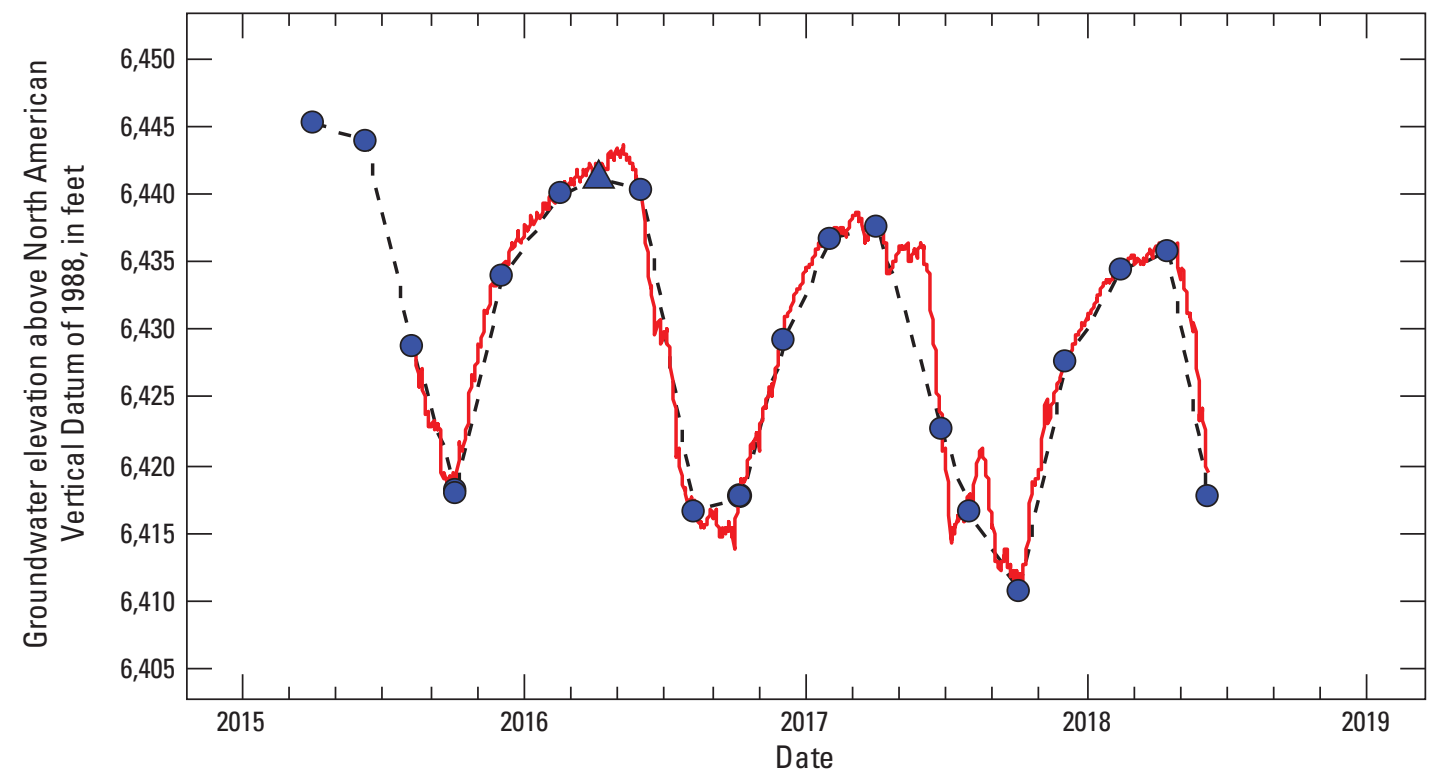

EXPLANATION

Continuous record groundwater level

- Daily maximum Groundwater level

$\triangle$ Rec ently pumped

O Static

Figure 2.24. Groundwater-level hydrograph for LDAW 12, USGS site number 392058104364401 , Elbert County, Colorado. 


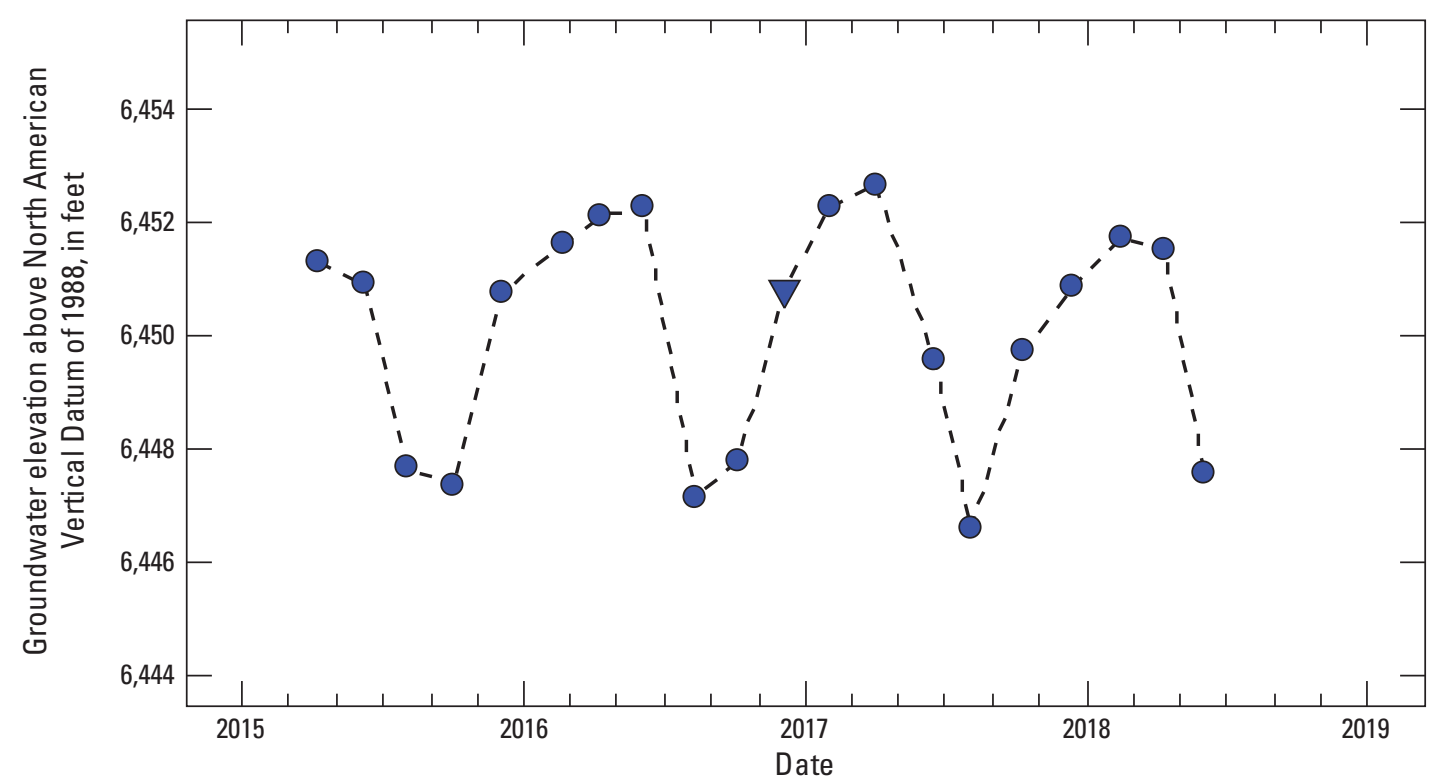

EXPLANATION

Groundwater level

$\nabla$ Nearby pumping

- Static

Figure 2.25. Groundwater-level hydrograph for LDAW 14, USGS site number 392125104323701 , Elbert County, Colorado.

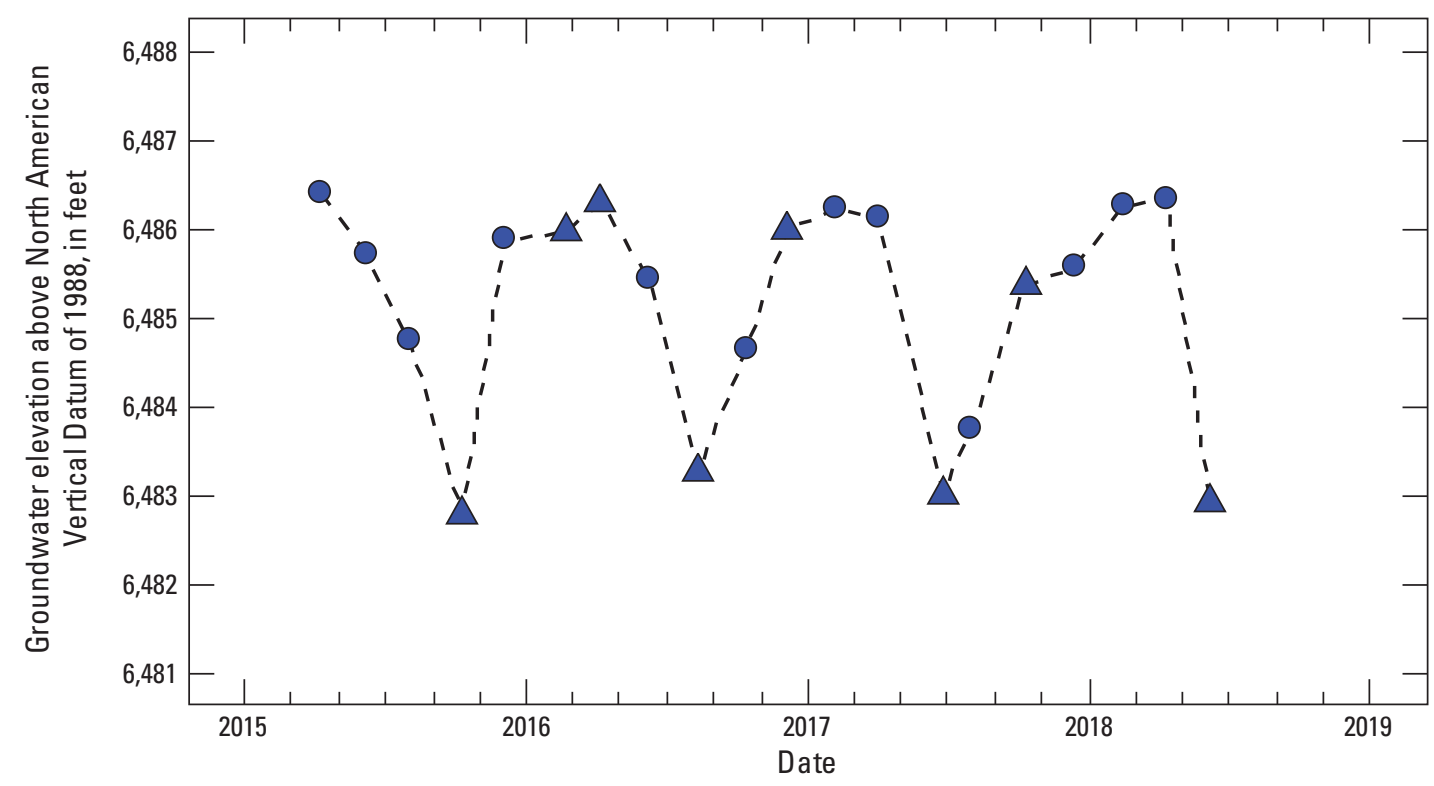

EXPLANATION

Groundwater level

$\triangle$ Recently pumped Static

Figure 2.26. Groundwater-level hydrograph for UDAW 17, USGS site number 392130104341401, Elbert County, Colorado. 


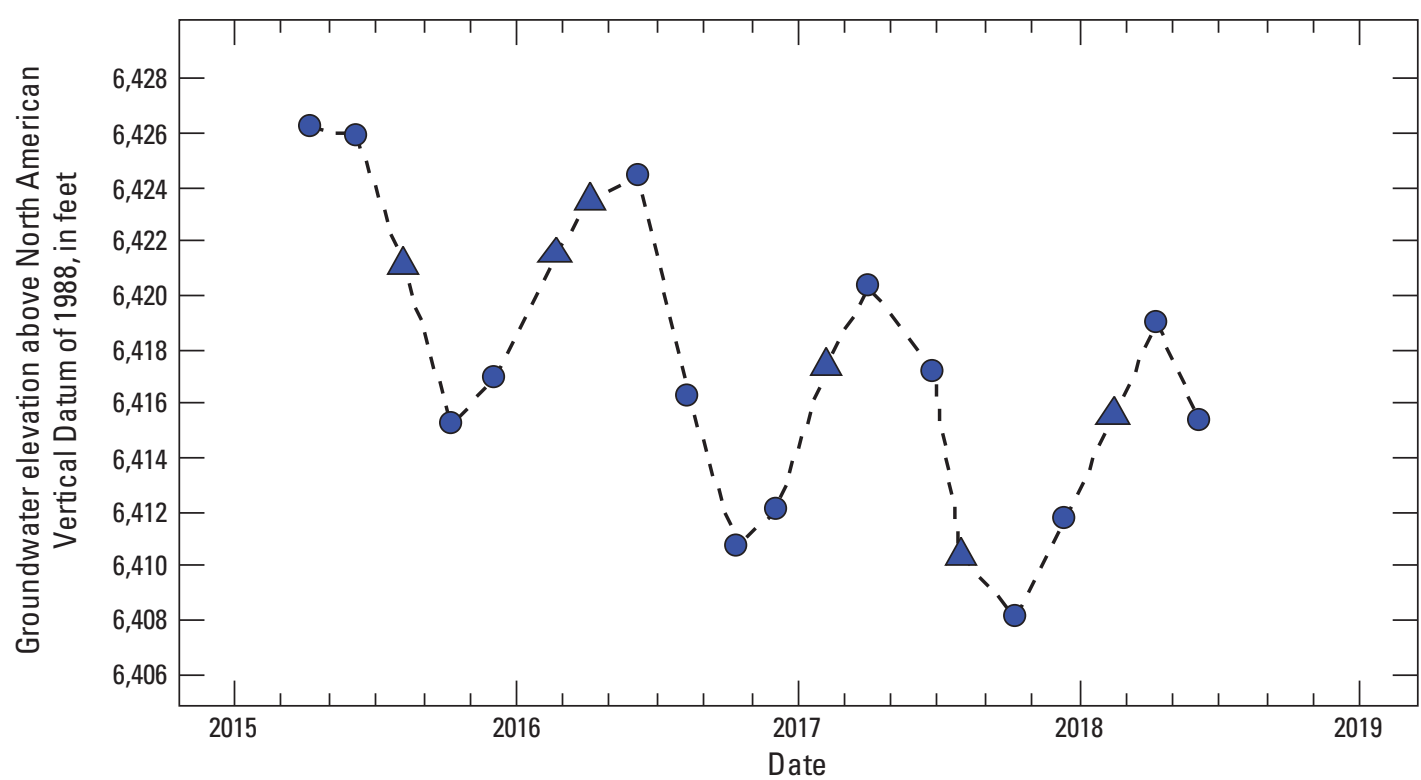

EXPLANATION

Groundwater level

$\triangle$ Recently pumped

Static

Figure 2.27. Groundwater-level hydrograph for DAWMAS21, USGS site number 392131104351701, Elbert County, Colorado.

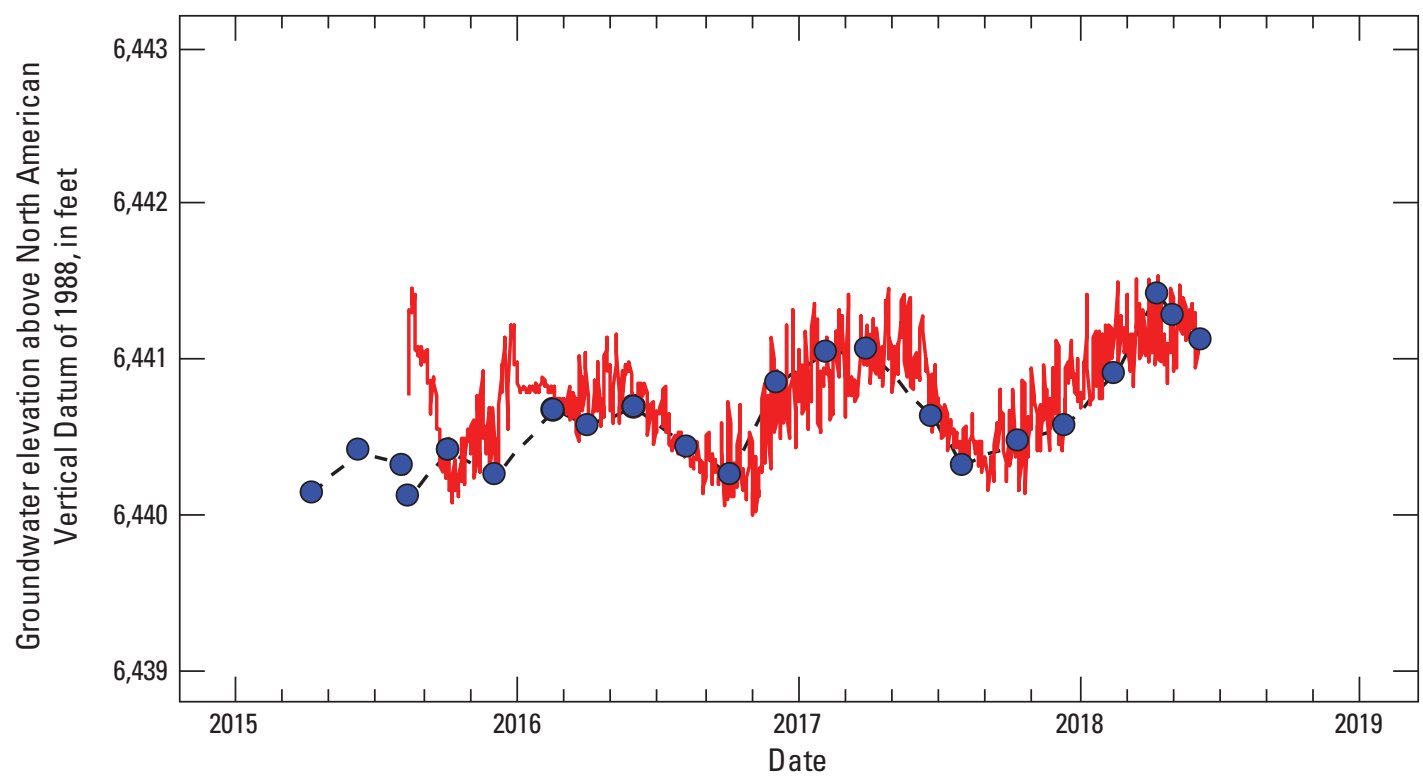

EXPLANATION

Continuous record groundwater level

- Daily maximum

Groundwater level

- Static

Figure 2.28. Groundwater-level hydrograph for UDAW 12, USGS site number 392133104310201 , Elbert County, Colorado. 


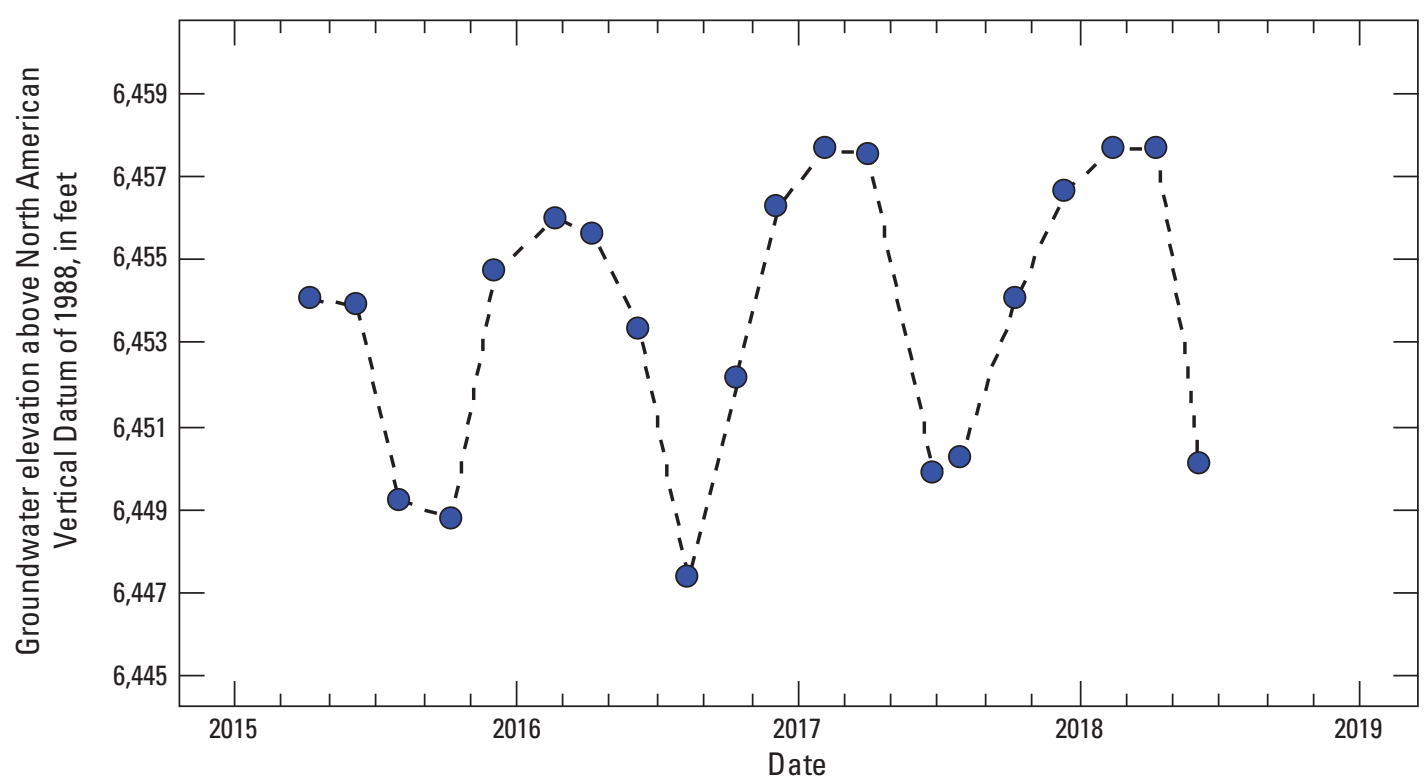

EXPLANATION

Groundwater level

- Static

Figure 2.29. Groundwater-level hydrograph for UDAW 16, USGS site number 392203104342301, Elbert County, Colorado.

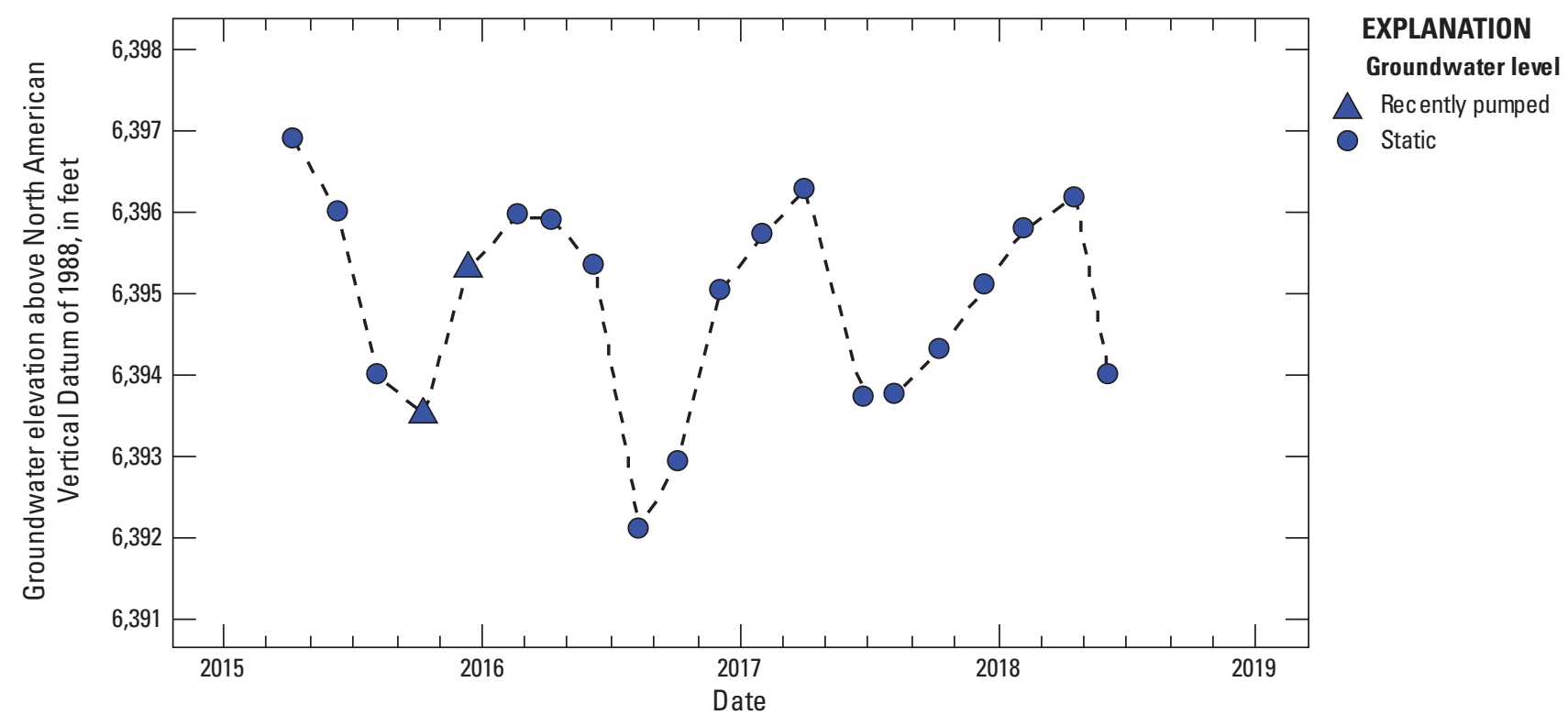

Figure 2.30. Groundwater-level hydrograph for UDAW 15, USGS site number 392355104382001, Elbert County, Colorado. 


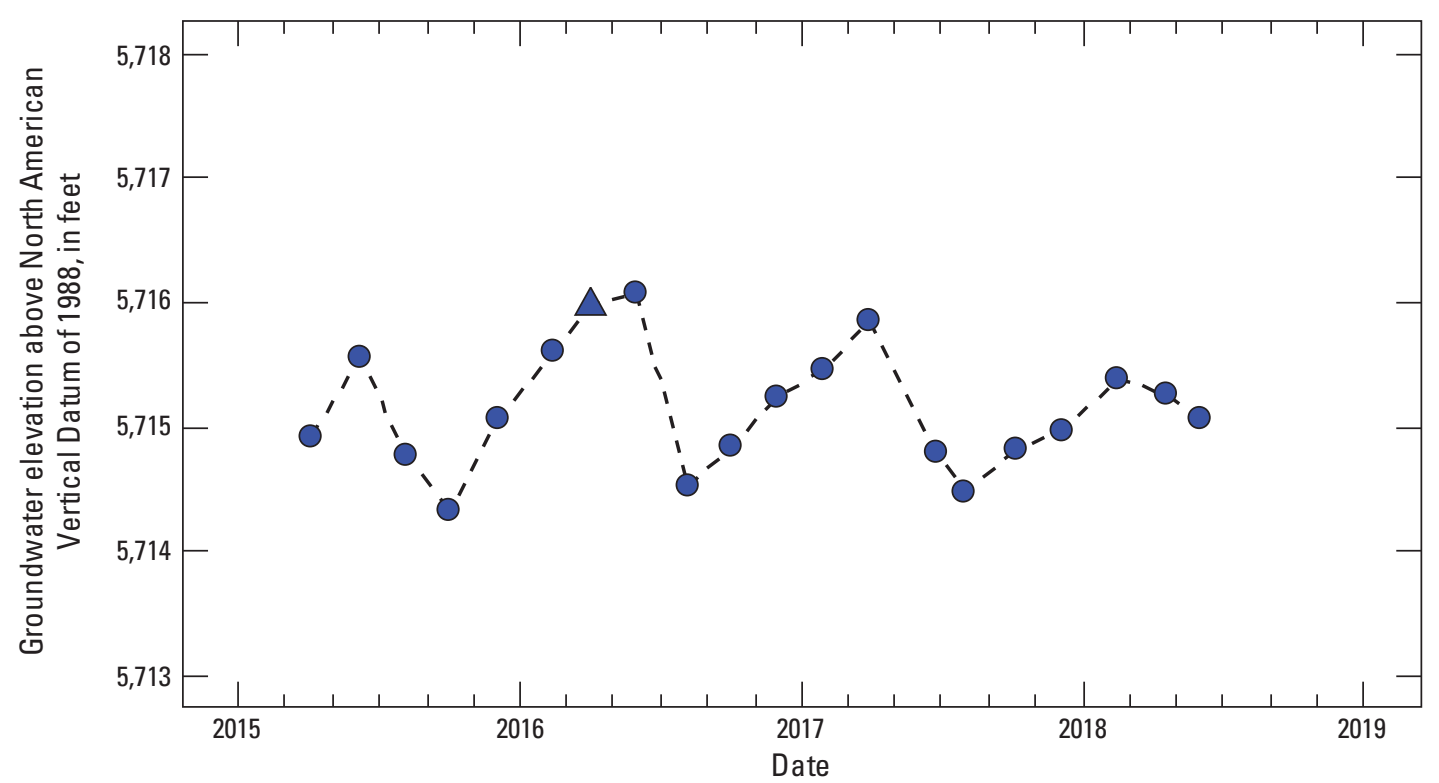

EXPLANATION

Groundwater level

$\triangle$ Rec ently pumped

- Static

Figure 2.31. Groundwater-level hydrograph for ARAPMAS28, USGS site number 392400104150601, Elbert County, Colorado.

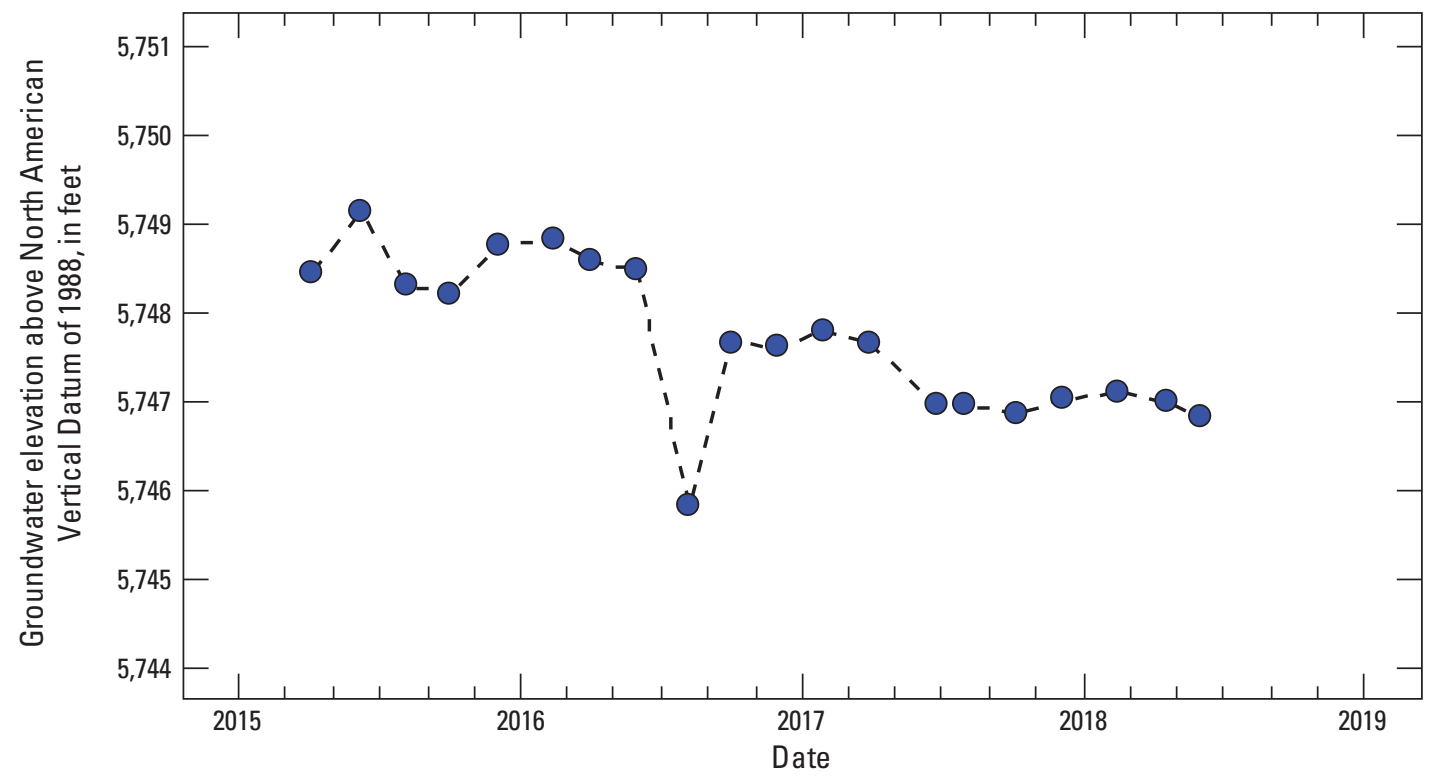

EXPLANATION

Groundwater level

Static

Figure 2.32. Groundwater-level hydrograph for ARAP 5, USGS site number 392434104142701, Elbert County, Colorado. 


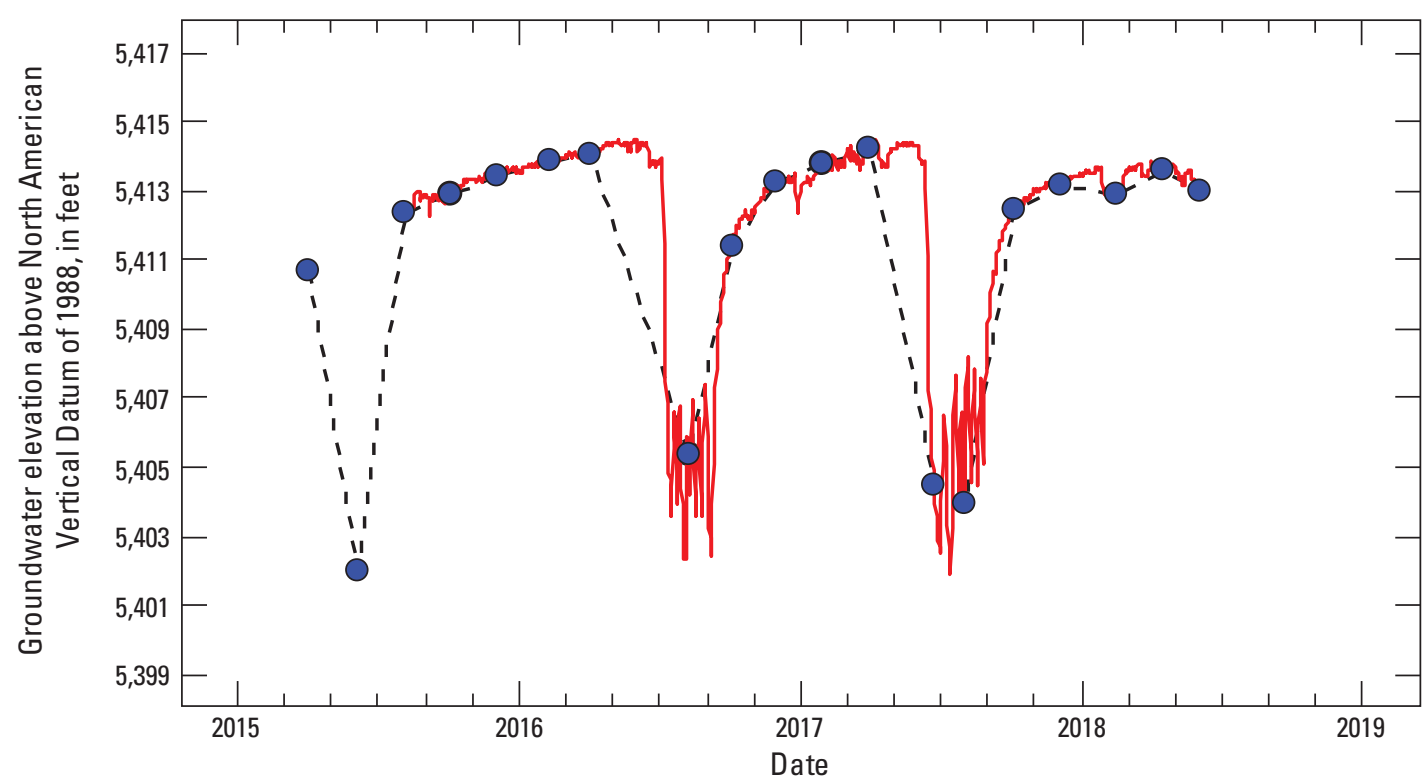

EXPLANATION

Continuous record groundwater level

- Daily maximum Groundwater level

- Static

Figure 2.33. Groundwater-level hydrograph for LARA 3, USGS site number 392616103591001, Elbert County, Colorado.

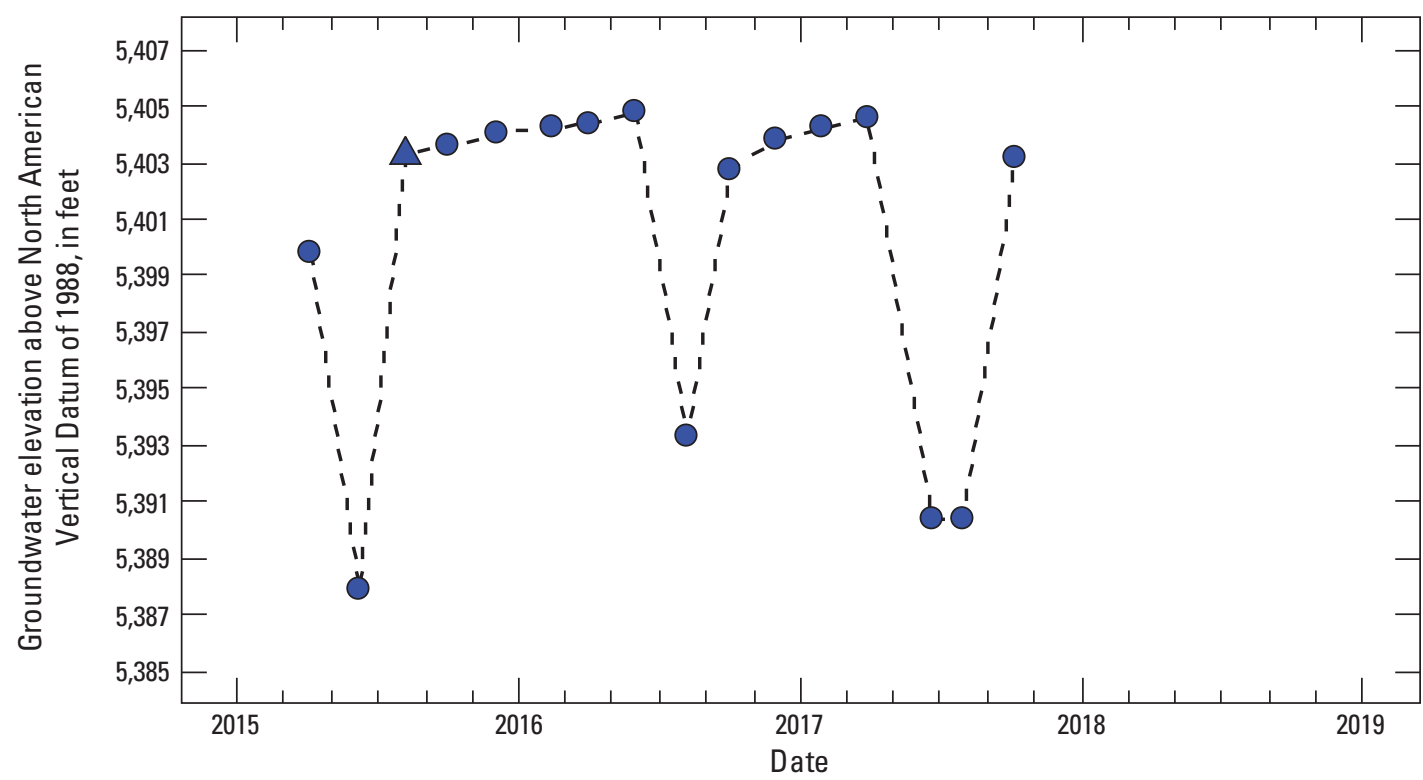

EXPLANATION

Ground water level

$\triangle$ Rec ently pumped

- Static

Figure 2.34. Groundwater-level hydrograph for LARA 4, USGS site number 392635103590001 , Elbert County, Colorado. 


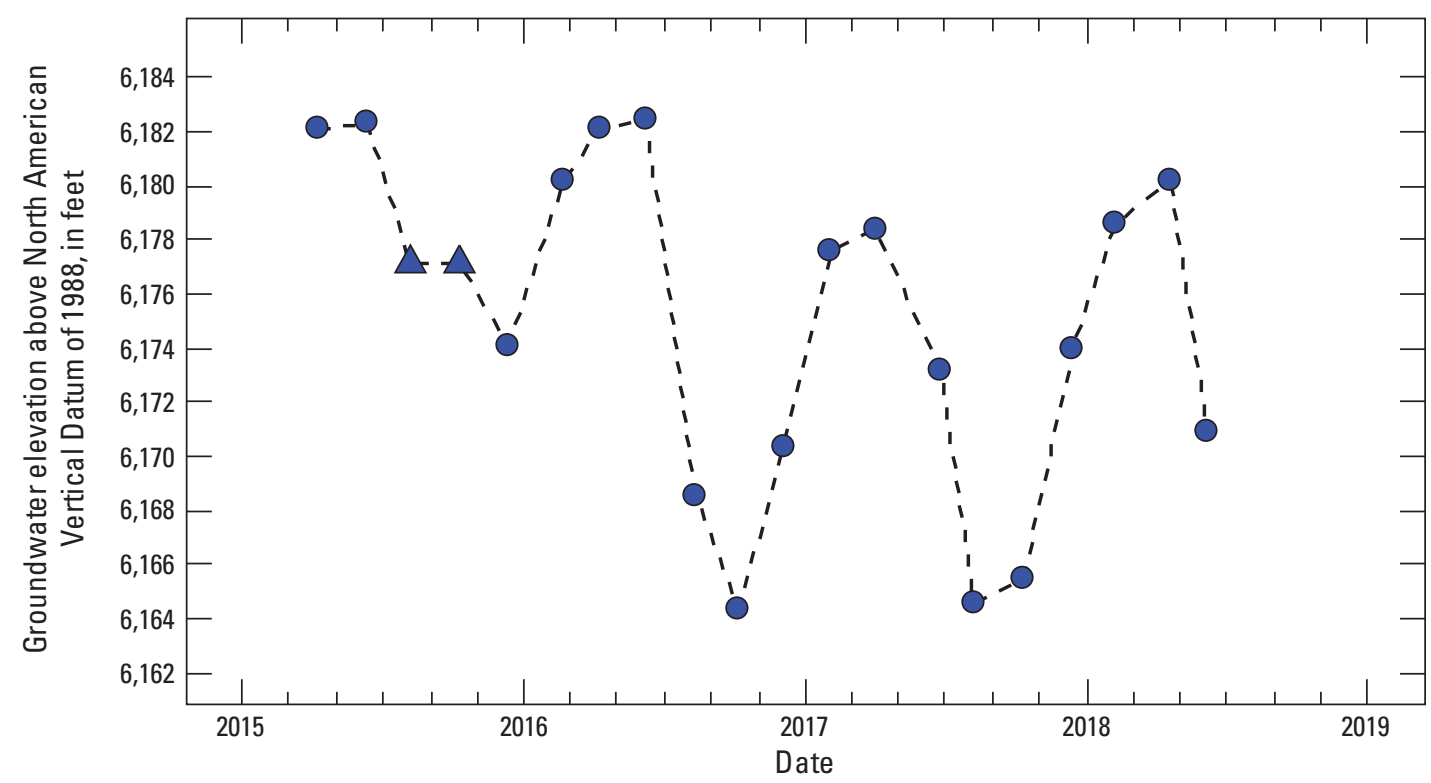

EXPLANATION

Ground water level

$\triangle$ Rec ently pumped

- Static

Figure 2.35. Groundwater-level hydrograph for LDAW 13, USGS site number 392724104341901, Elbert County, Colorado.

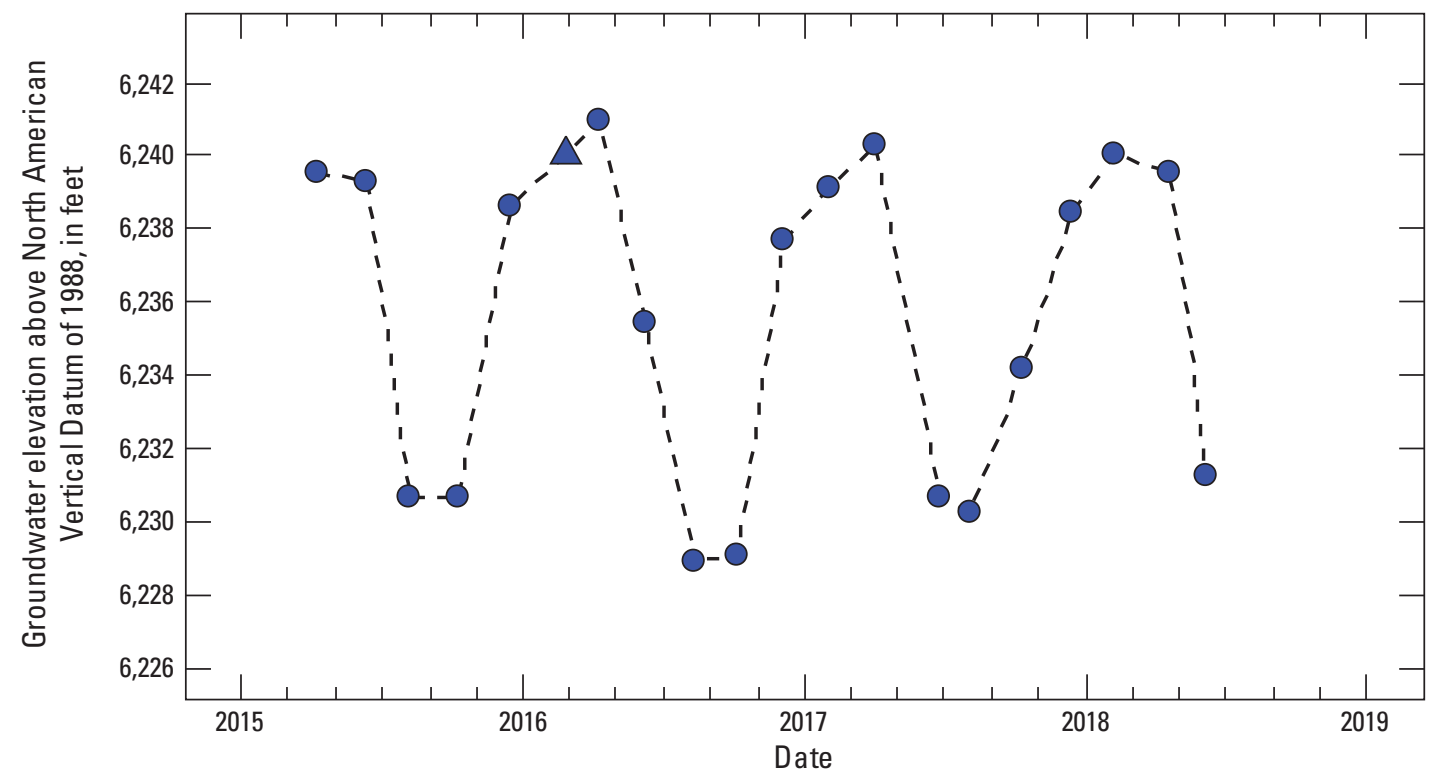

EXPLANATION

Groundwater level

$\triangle$ Rec ently pumped

- Static

Figure 2.36. Groundwater-level hydrograph for UDAW 13, USGS site number 392856104393801, Elbert County, Colorado. 


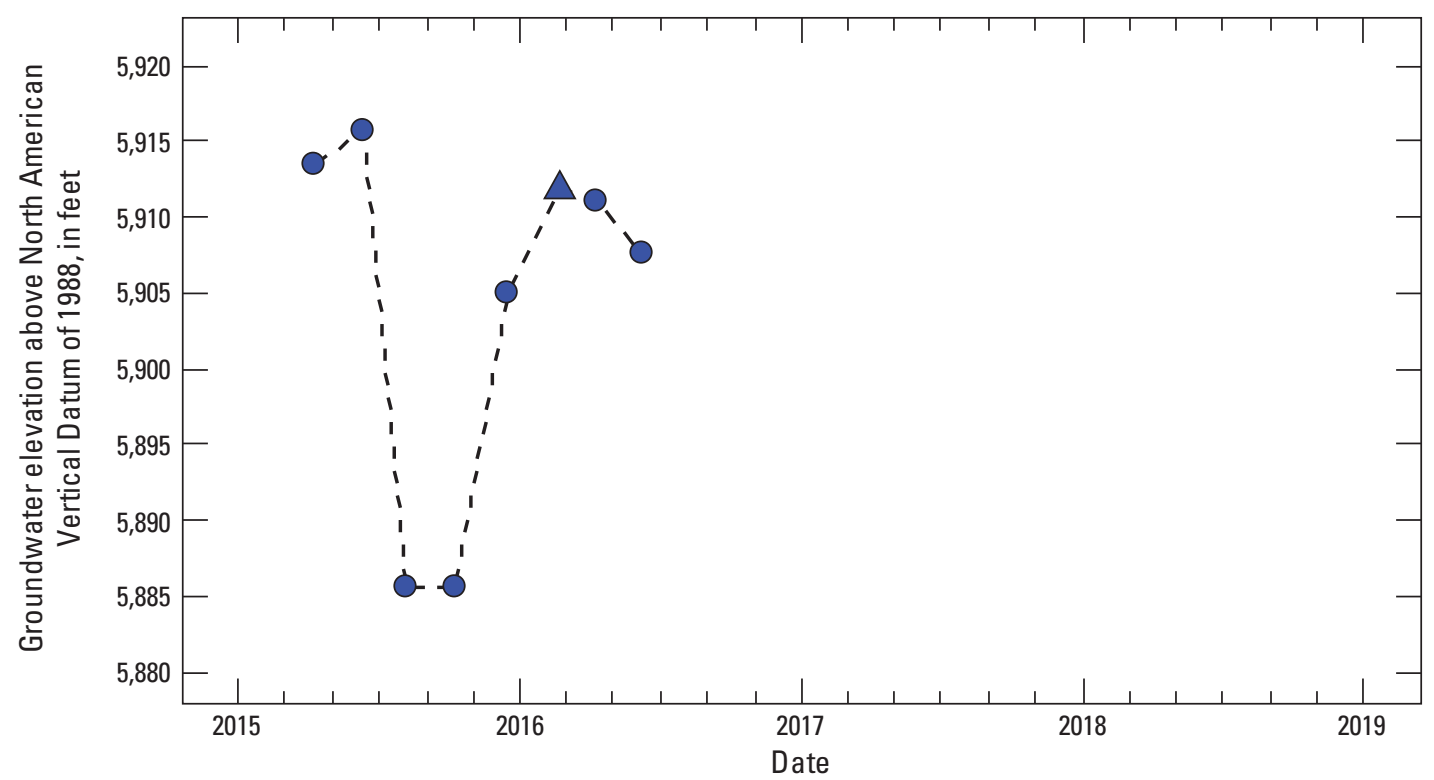

EXPLANATION Groundwater level $\triangle$ Rec ently pumped - Static

Figure 2.37. Groundwater-level hydrograph for DENV 13, USGS site number 393012104310701 , Elbert County, Colorado.

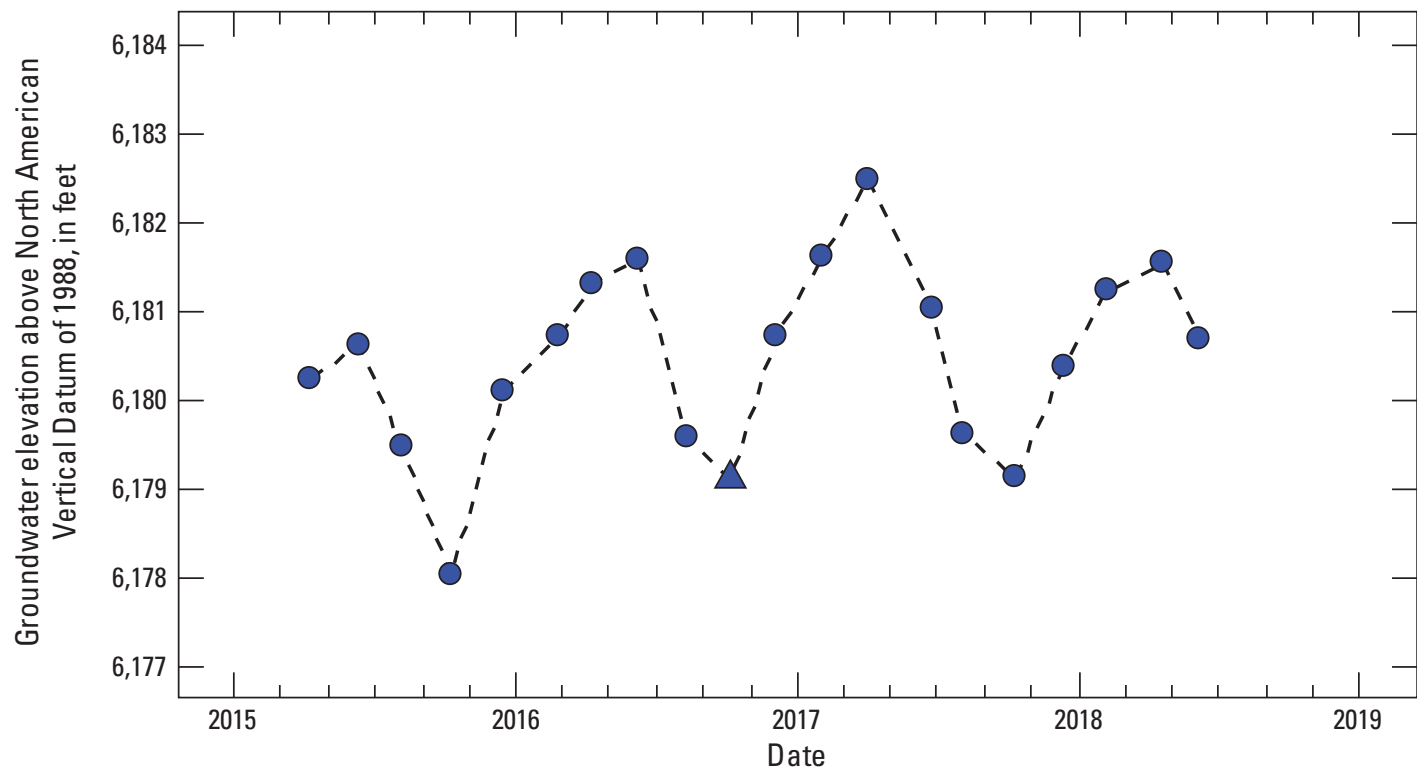

$\triangle$ Rec ently pumped

Static

Figure 2.38. Groundwater-level hydrograph for UDAW 11, USGS site number 393016104392601 , Elbert County, Colorado. 


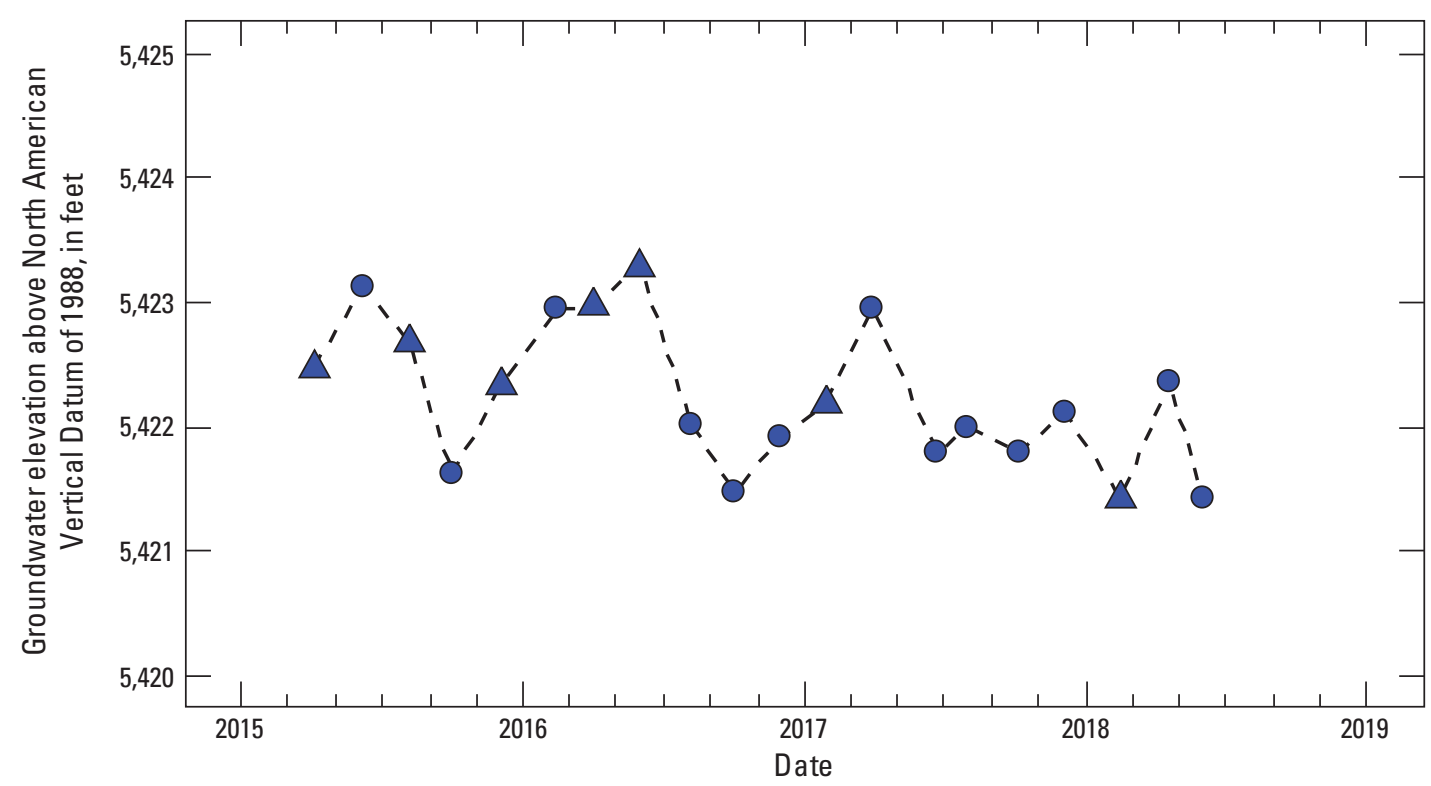

\section{EXPLANATION}

Groundwater level

$\triangle$ Rec ently pumped

- Static

Figure 2.39. Groundwater-level hydrograph for ARAP 4, USGS site number 393225104073601 , Elbert County, Colorado.

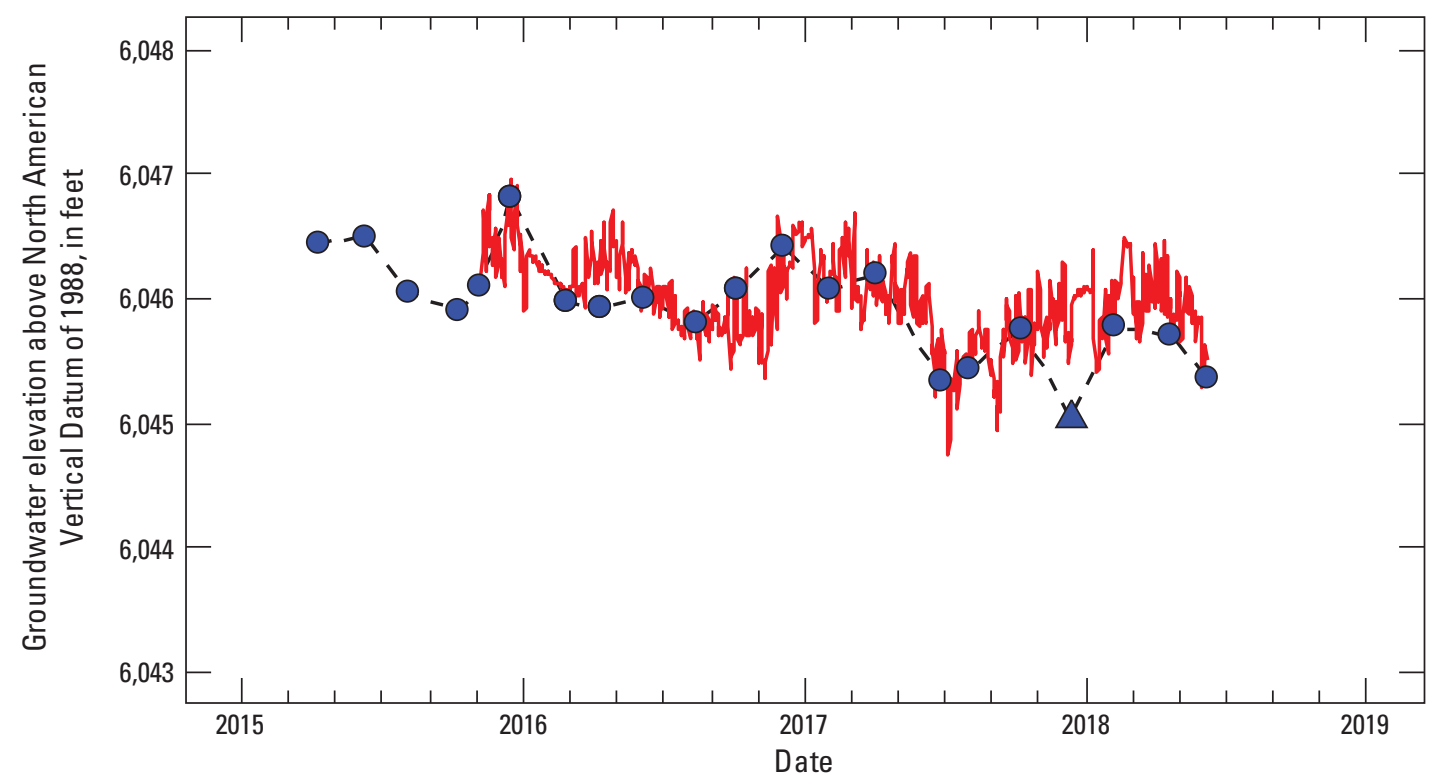

EXPLANATION

Continuous record ground water level

- Daily maximum Ground water level

$\triangle$ Recently pumped

- Static

Figure 2.40. Groundwater-level hydrograph for DAWMAS19, USGS site number 393227104343401, Elbert County, Colorado. 


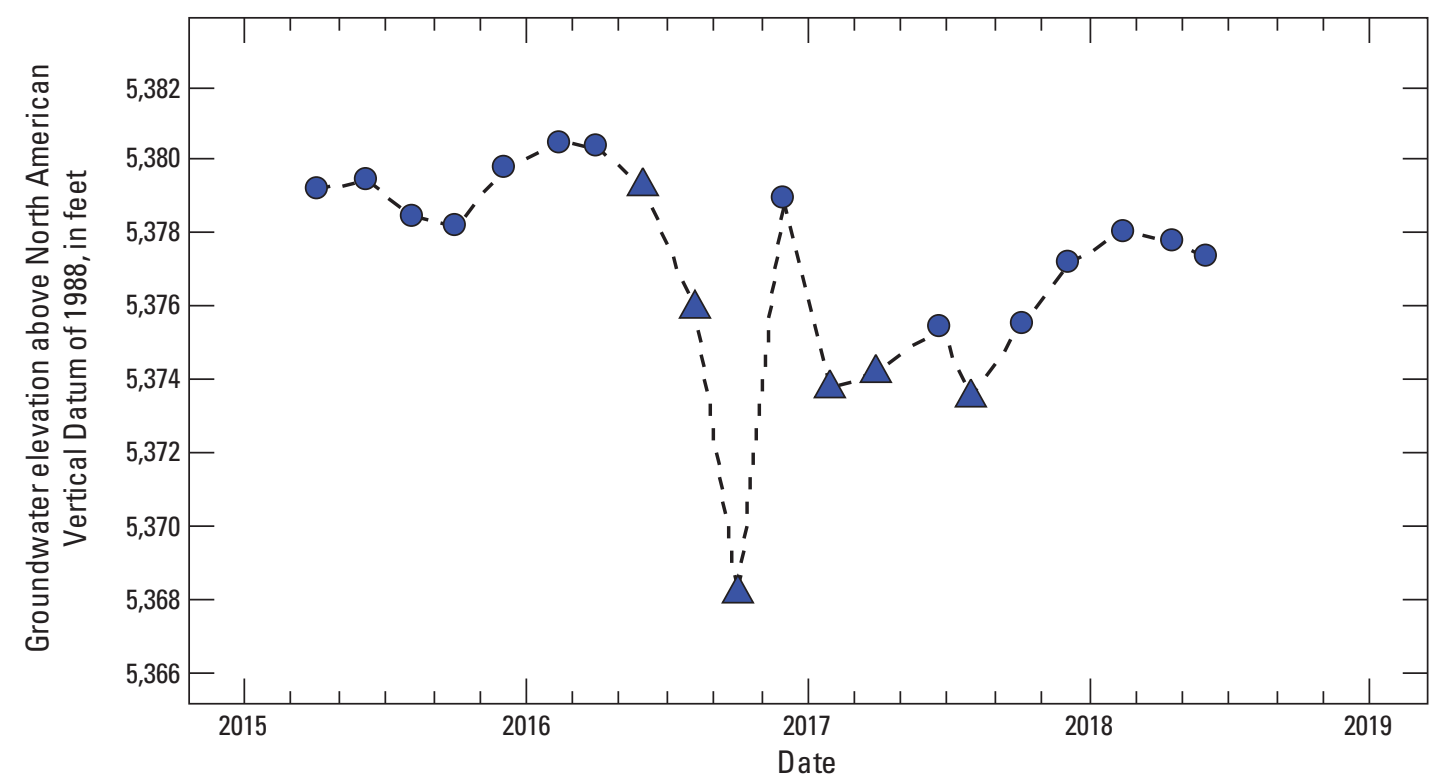

EXPLANATION

Groundwater level

$\triangle$ Recently pumped

- Static

Figure 2.41. Groundwater-level hydrograph for ARAP 3, USGS site number 393251104073701, Elbert County, Colorado.

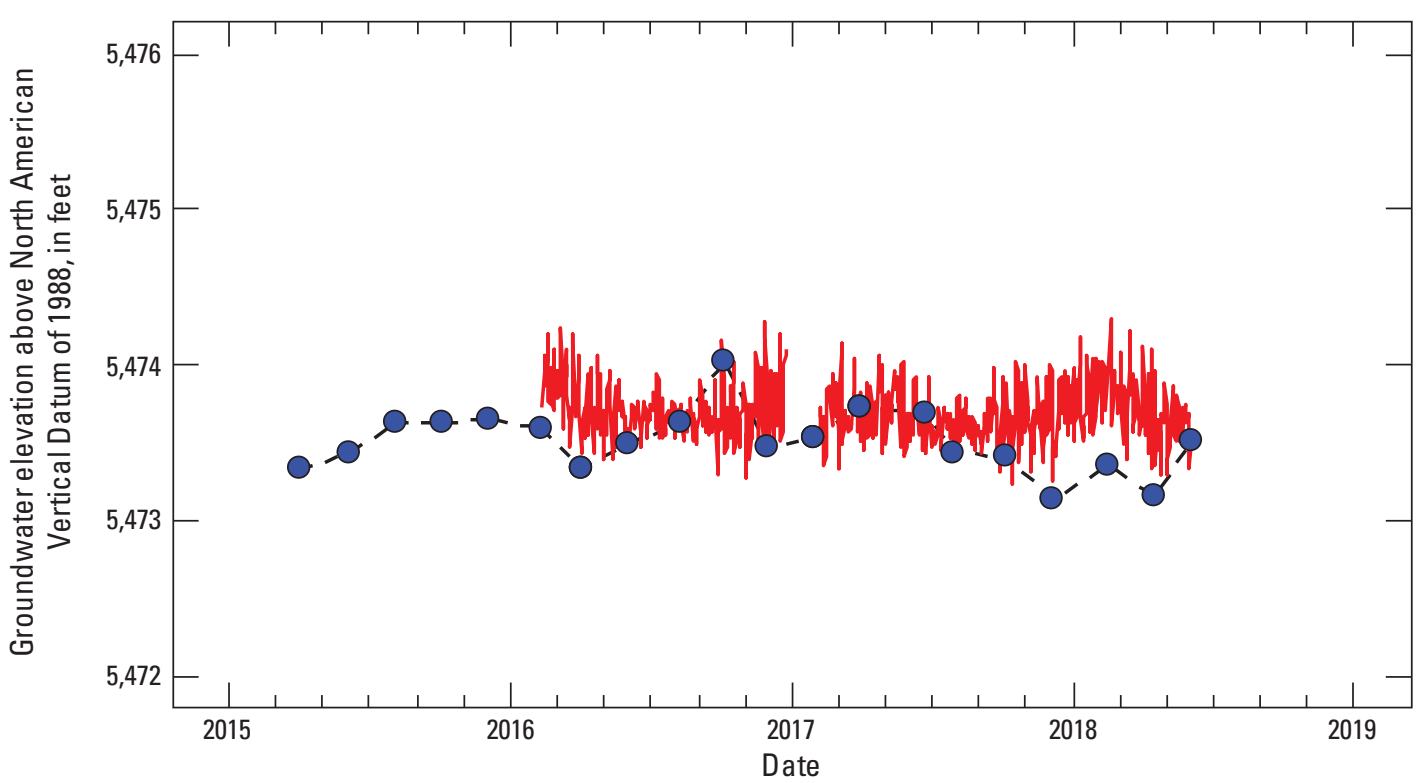

EXPLANATION

Continuous record groundwater level

- Daily maximum Groundwater level

- Static

Figure 2.42. Groundwater-level hydrograph for DENV 12, USGS site number 393350104151701 , Elbert County, Colorado. 


\section{Appendix 3. Discrete Groundwater-Level Elevation Trends}

Table 3.1. Trends in discrete static groundwater-level elevations, April 2015 to June 2018, Elbert County, Colorado.

[See table 2 and figure 2 for well locations. ft, foot; $p$-value, probability value; $\mathrm{R}^{2}$, coefficient of determination; UDAW, upper Dawson aquifer; LDAW, lower Dawson aquifer; DENV, Denver aquifer; ARAP, Arapahoe aquifer; LARA, Laramie-Fox Hills aquifer; Lin. Reg., Linear Regression; Lin. Reg. Seas., Linear Regression with Seasonality terms; --, insufficient data to compute trend; $<$, less than]

\begin{tabular}{|c|c|c|c|c|c|c|}
\hline $\begin{array}{c}\text { Site } \\
\text { identification } \\
\text { number }\end{array}$ & Aquifer & $\begin{array}{c}\text { Common } \\
\text { name }\end{array}$ & $\begin{array}{c}\text { Trend } \\
\text { test }\end{array}$ & $\begin{array}{c}\text { Trend in } \\
\text { grounwater } \\
\text { level (ft/year) }\end{array}$ & $\begin{array}{l}\text { Trend } \\
\text { slope } \\
p \text {-value }\end{array}$ & $\begin{array}{c}\text { Linear } \\
\text { regression } \\
\mathbf{R}^{2}\end{array}$ \\
\hline 390935104301001 & UDAW & DAWMAS26 & Lin. Reg. & -0.01 & 0.943 & -0.10 \\
\hline \multirow{2}{*}{391126104354701} & \multirow{2}{*}{ UDAW } & \multirow{2}{*}{ UDAW 19} & Lin. Reg. & 0.15 & 0.043 & 0.20 \\
\hline & & & Lin. Reg. Seas. & 0.15 & 0.004 & 0.69 \\
\hline \multirow{2}{*}{391915104375001} & \multirow{2}{*}{ UDAW } & \multirow{2}{*}{ UDAW 18} & Lin. Reg. & -0.26 & 0.592 & -0.09 \\
\hline & & & Lin. Reg. Seas. & -0.53 & 0.186 & 0.58 \\
\hline \multirow{2}{*}{391924104374101} & \multirow{2}{*}{ UDAW } & \multirow{2}{*}{ UDAW 14} & Lin. Reg. & -0.17 & 0.498 & -0.02 \\
\hline & & & Lin. Reg. Seas. & -0.26 & 0.045 & 0.75 \\
\hline 392130104341401 & UDAW & UDAW 17 & Lin. Reg. & 0.03 & 0.894 & -0.10 \\
\hline 392203104342301 & UDAW & UDAW 16 & Lin. Reg. Seas. & 0.45 & 0.236 & 0.78 \\
\hline & UDAW & UDAW 15 & Lin. Reg. & -0.24 & 0.470 & -0.03 \\
\hline 392355104382001 & UDAW & UDAW 15 & Lin. Reg. Seas. & -0.24 & 0.168 & 0.75 \\
\hline 200056101202001 & IDAU & HDAW 12 & Lin. Reg. & -0.07 & 0.948 & -0.06 \\
\hline 392856104393801 & UDAW & UDAW 13 & Lin. Reg. Seas. & -0.70 & 0.146 & 0.82 \\
\hline 202016101202601 & IDAW & JDAW 11 & Lin. Reg. & 0.31 & 0.206 & 0.04 \\
\hline 393010104392001 & UDAW & UDAW 11 & Lin. Reg. Seas. & 0.28 & 0.056 & 0.70 \\
\hline 201148104204101 & IDAW & DАUМ 227 & Lin. Reg. & 0.15 & 0.237 & 0.03 \\
\hline 391148104294101 & LDAW & DAWIMASLI & Lin. Reg. Seas. & 0.13 & 0.005 & 0.91 \\
\hline 301502104273601 & IDAW & IDAW 16 & Lin. Reg. & -0.21 & 0.675 & -0.19 \\
\hline & & & Lin. Reg. & -3.23 & 0.188 & 0.04 \\
\hline 392058104364401 & LDAW & LDAW 12 & Lin. Reg. Seas. & -4.91 & $<0.001$ & 0.86 \\
\hline 392125104323701 & I DAW & IDAW 14 & Lin. Reg. & -0.03 & 0.944 & -0.06 \\
\hline $392125104323 / 01$ & LDAW & LDAW 14 & Lin. Reg. Seas. & -0.25 & 0.346 & 0.71 \\
\hline 202121104251701 & $\mathrm{I} \cap \mathrm{W}$ & DАШМ & Lin. Reg. & -2.91 & 0.048 & 0.23 \\
\hline $392131104351 / 01$ & LDAW & DAWIMASZI & Lin. Reg. Seas. & -3.16 & $<0.001$ & 0.96 \\
\hline 392724104341901 & IDAW & LAW 13 & Lin. Reg. & -2.65 & 0.104 & 0.10 \\
\hline & LDAW & LDAW 13 & Lin. Reg. Seas. & -1.88 & 0.0120 & 0.84 \\
\hline & & DAWMAS 19 & Lin. Reg. & -0.28 & $<0.001$ & 0.41 \\
\hline 393227104343401 & LDAW & DAWMAS19 & Lin. Reg. Seas. & -0.25 & $<0.001$ & 0.59 \\
\hline
\end{tabular}


Table 3.1. Trends in discrete static groundwater-level elevations, April 2015 to June 2018, Elbert County, Colorado.—Continued

[See table 2 and figure 2 for well locations. ft, foot; $p$-value, probability value; $\mathrm{R}^{2}$, coefficient of determination; UDAW, upper Dawson aquifer; LDAW, lower Dawson aquifer; DENV, Denver aquifer; ARAP, Arapahoe aquifer; LARA, Laramie-Fox Hills aquifer; Lin. Reg., Linear Regression; Lin. Reg. Seas., Linear Regression with Seasonality terms; --, insufficient data to compute trend; $<$, less than]

\begin{tabular}{|c|c|c|c|c|c|c|}
\hline $\begin{array}{c}\text { Site } \\
\text { identification } \\
\text { number }\end{array}$ & Aquifer & $\begin{array}{c}\text { Common } \\
\text { name }\end{array}$ & $\begin{array}{c}\text { Trend } \\
\text { test }\end{array}$ & $\begin{array}{c}\text { Trend in } \\
\text { grounwater } \\
\text { level (ft/year) }\end{array}$ & $\begin{array}{c}\text { Trend } \\
\text { slope } \\
p \text {-value }\end{array}$ & $\begin{array}{c}\text { Linear } \\
\text { regression } \\
\mathbf{R}^{2} \\
\end{array}$ \\
\hline \multirow{2}{*}{390755104172501} & \multirow{2}{*}{ DENV } & \multirow{2}{*}{ DENV 17} & Lin. Reg. & -60.02 & -- & -- \\
\hline & & & Lin. Reg. Seas. & -60.02 & -- & -- \\
\hline \multirow{2}{*}{391257104173601} & \multirow{2}{*}{ DENV } & \multirow{2}{*}{ DENV 16} & Lin. Reg. & 0.01 & 0.733 & -0.05 \\
\hline & & & Lin. Reg. Seas. & 0.01 & 0.829 & -0.12 \\
\hline \multirow{2}{*}{391811104140301} & \multirow{2}{*}{ DENV } & \multirow{2}{*}{ DENV 15} & Lin. Reg. & -0.16 & 0.297 & 0.01 \\
\hline & & & Lin. Reg. Seas. & -0.18 & 0.148 & 0.41 \\
\hline \multirow{2}{*}{391821104270601} & \multirow{2}{*}{ DENV } & \multirow{2}{*}{ DENV 14} & Lin. Reg. & -1.12 & 0.017 & 0.34 \\
\hline & & & Lin. Reg. Seas. & -1.04 & 0.005 & 0.67 \\
\hline \multirow{2}{*}{391851104204501} & \multirow{2}{*}{ DENV } & \multirow{2}{*}{ DENMAS05 } & Lin. Reg. & 1.44 & 0.444 & -0.03 \\
\hline & & & Lin. Reg. Seas. & 1.08 & 0.574 & -0.05 \\
\hline \multirow{2}{*}{393012104310701} & \multirow{2}{*}{ DENV } & \multirow{2}{*}{ DENV 13} & Lin. Reg. & 0.96 & 0.945 & -0.20 \\
\hline & & & Lin. Reg. Seas. & -1.55 & 0.861 & 0.55 \\
\hline \multirow{2}{*}{393350104151701} & \multirow{2}{*}{ DENV } & \multirow{2}{*}{ DENV 12} & Lin. Reg. & -0.05 & 0.307 & 0.00 \\
\hline & & & Lin. Reg. Seas. & -0.04 & 0.354 & 0.01 \\
\hline \multirow{2}{*}{390800104172601} & \multirow{2}{*}{ ARAP } & \multirow{2}{*}{ ARAP 8} & Lin. Reg. & -0.17 & 0.704 & -0.12 \\
\hline & & & Lin. Reg. Seas. & 0.56 & 0.323 & 0.38 \\
\hline 391208104053301 & $A R A P$ & $\mathrm{ARAP} 7$ & Lin. Reg. & -0.83 & 0.060 & 0.16 \\
\hline $59120010+050501$ & Нก & НINAा 1 & Lin. Reg. Seas. & -0.79 & 0.087 & 0.12 \\
\hline 391740104072401 & $A R A P$ & ARAPMAS27 & Lin. Reg. & 0.38 & $<0.001$ & 0.87 \\
\hline 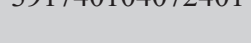 & ค1 & 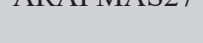 & Lin. Reg. Seas. & 0.38 & $<0.001$ & 0.87 \\
\hline 391834104205601 & $\mathrm{ARAP}$ & AR APMAS?2 & Lin. Reg. & -0.63 & 0.017 & 0.30 \\
\hline 391034104205001 & Нก & ANAI VIADEL & Lin. Reg. Seas. & -0.73 & 0.001 & 0.63 \\
\hline 391946104114501 & $\mathrm{ARAP}$ & $\mathrm{ARAP} 6$ & Lin. Reg. & 0.38 & 0.314 & 0.00 \\
\hline 19 & 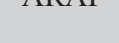 & ת & Lin. Reg. Seas. & 0.36 & 0.326 & 0.05 \\
\hline 392400104150601 & ARAP & ARAPMAS28 & Lin. Reg. & 0.01 & 0.931 & -0.06 \\
\hline 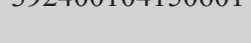 & (2) & 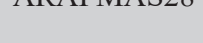 & Lin. Reg. Seas. & -0.03 & 0.719 & 0.43 \\
\hline 392434104142701 & $A R A P$ & $\mathrm{ARAP} 5$ & Lin. Reg. & -0.66 & $<0.001$ & 0.55 \\
\hline 19 & ת & ת10ता & Lin. Reg. Seas. & -0.69 & $<0.001$ & 0.66 \\
\hline 393225104073601 & ARAP & $\mathrm{ARAP} 4$ & Lin. Reg. & -0.23 & 0.193 & 0.07 \\
\hline 19 & (2) & ת & Lin. Reg. Seas. & -0.30 & 0.020 & 0.63 \\
\hline 393251104073701 & ARAP & $\mathrm{ARAP} 3$ & Lin. Reg. & -0.87 & 0.012 & 0.37 \\
\hline 10 & (110 & 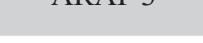 & Lin. Reg. Seas. & -0.94 & 0.001 & 0.70 \\
\hline 390817104040301 & $\mathrm{LARA}$ & $\mathrm{IARA} 7$ & Lin. Reg. & -0.74 & 0.014 & 0.41 \\
\hline 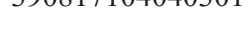 & 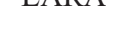 & Lनित & Lin. Reg. Seas. & -0.54 & 0.043 & 0.58 \\
\hline 391609104014001 & LARA & LARA 6 & Lin. Reg. & -0.70 & $<0.001$ & 0.66 \\
\hline 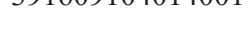 & Lसा & 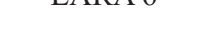 & Lin. Reg. Seas. & -0.73 & $<0.001$ & 0.84 \\
\hline 391621104012001 & LARA & LARA 5 & Lin. Reg. & -0.65 & $<0.001$ & 0.66 \\
\hline 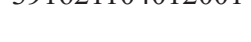 & Lथा & 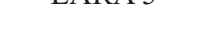 & Lin. Reg. Seas. & -0.69 & $<0.001$ & 0.88 \\
\hline 392616103591001 & LARA & $\mathrm{IARA} 3$ & Lin. Reg. & 0.52 & 0.566 & -0.03 \\
\hline 10 & Lसा & 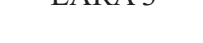 & Lin. Reg. Seas. & 0.33 & 0.626 & 0.44 \\
\hline 202625102500001 & $\mathrm{I} A \mathrm{PA}$ & $I \wedge P \wedge \triangle>>$ & Lin. Reg. & -0.38 & 0.866 & -0.07 \\
\hline 392033103390001 & LAKA & LAKA 4 & Lin. Reg. Seas. & 0.35 & 0.839 & 0.43 \\
\hline
\end{tabular}





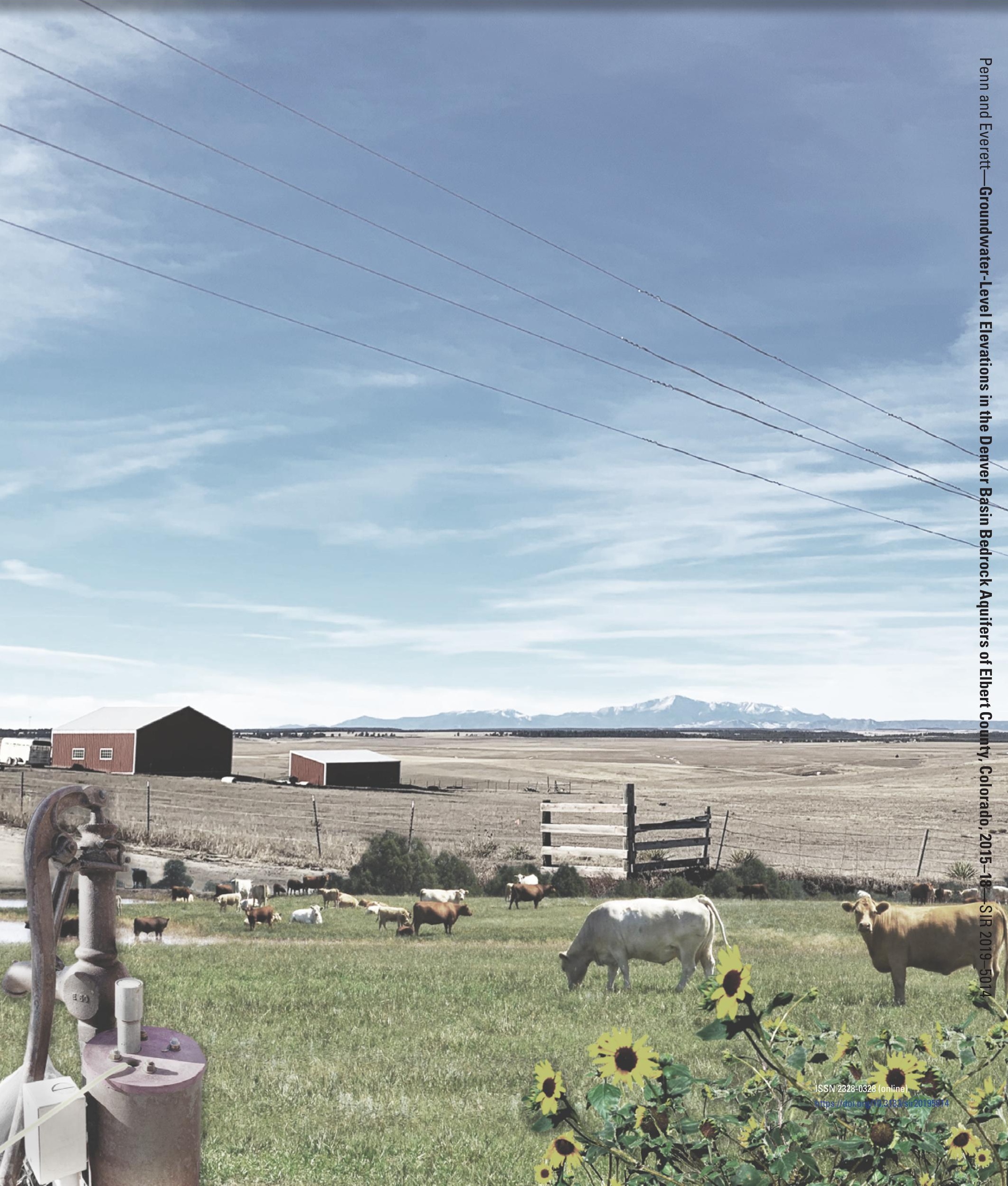

